Cointegração fracionária em séries financeiras

Victor Sakimoto Shie

DISSERTAÇÃO APRESENTADA

$\mathrm{AO}$

INSTITUTO DE MATEMÁTICA E ESTATÍSTICA

DA

UNIVERSIDADE DE SÃO PAULO

PARA

OBTENÇÃO DO TÍTULO

DE MESTRE EM CIÊNCIAS

Programa: Estatística

Orientadora: Profa. Dra. Chang Chiann

São Paulo, maio de 2010 


\section{Cointegração fracionária em séries financeiras}

Este exemplar corresponde à redação

final da dissertação devidamente corrigida

e defendida por Victor Sakimoto Shie

e aprovada pela Comissão Julgadora.

Banca Examinadora:

- Profa. Dra. Chang Chiann (orientadora) - IME - USP.

- Prof. Dr. Airlane Pereira Alencar - IME - USP.

- Prof. Dr. Guilherme de Oliveira Lima Cagliari Marques - CECS - UFABC. 


\section{Agradecimentos}

Agradeço à minha orientadora Profa. Dra. Chang Chiann por todos os ensinamentos, a amizade e o auxílio que foram fundamentais para a conclusão deste trabalho.

À minha família, amigos e à Kelly pelo apoio, motivação e companherismo.

Ao Prof. Dr. Guilherme de Oliveira Lima Cagliari Marques e à Grazielle Yumi Soldá pelas ajudas prestadas.

À LAN House Monkey pelo apoio com as inúmeras e demoradas simulações.

Aos professores e colegas do IME pelos ensinamentos, amizade e paciência. 


\section{Resumo}

O objetivo deste trabalho é apresentar alguns testes de cointegração fracionária para séries integradas de ordem $d(d \in R)$, i.e., séries $I(d)$, comparando-os com os testes de cointegração, cujo parâmetro $d$ assume valores inteiros. O procedimento para os testes de cointegração fracionária utiliza reamostragens de bootstrap com reposição para gerar séries sob a hipótese nula de não cointegração. Estas reamostragens são então utilizadas para calcular os p-valores de algumas estatísticas de testes de regressão, tais como a estatística de Durbin-Watson e a estimativa do parâmetro de memória longa $(d)$ residual. O poder destes testes é apresentado e comparado com os testes de cointegração, mostrando sua consistência. A aplicação destes testes a dados reais compara o modelo de correção de erros de cointegração com o modelo de correção de erros de cointegração fracionária utilizando a medida de erros quadráticos médios dos modelos ajustados. 


\begin{abstract}
The purpose of this project is to present some fractional cointegration tests for integrated time series of order $d(d \in R)$, i.e., $I(d)$ time series, comparing them to cointegration tests, where the parameter $d$ assumes integer values. The tests' procedure is done by using bootstrap samples to obtain series under the null hypothesis of non-cointegration. These samples are then used to estimate the p-value of some regression-based test statistics, such as the Durbin-Watson statistic and estimates of residual $d$ parameter. The application of these tests to real series compares the error correction model of cointegration to the error correction model of fractional cointegration by evaluating the mean squared errors over the residuals from the fitted models.
\end{abstract}




\section{Sumário}

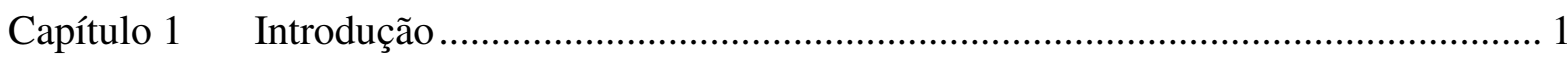

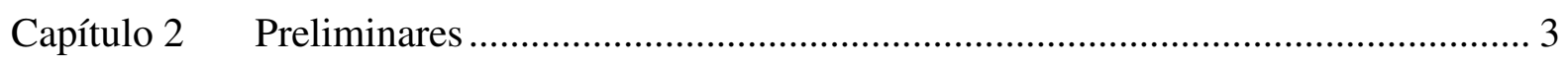

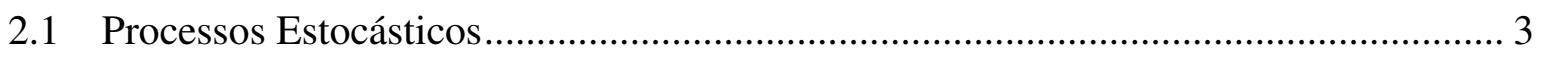

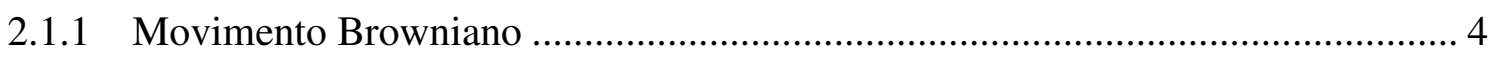

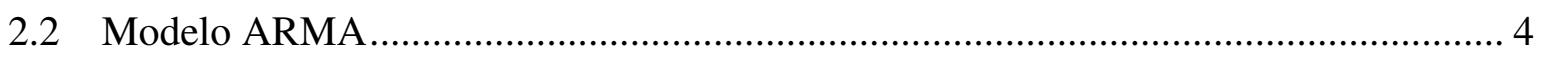

2.2.1 Modelo auto-regressivo (AR) .................................................................. 5

2.2.2 Modelo de médias móveis (MA)_................................................................... 6

2.2.3 Modelo auto-regressivo e de médias móveis ....................................................... 6

2.3 Séries Temporais não Estacionárias ………………………………………....... 7

2.3.1 Modelo ARIMA ……………………………….......................................... 7

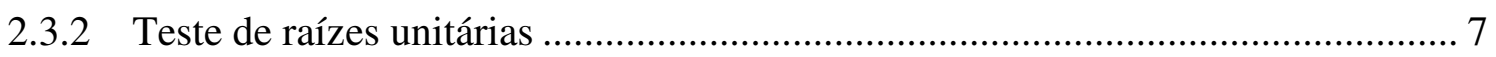

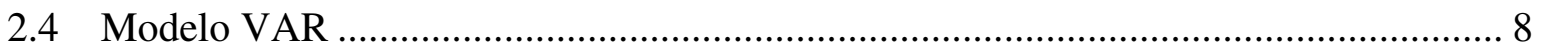

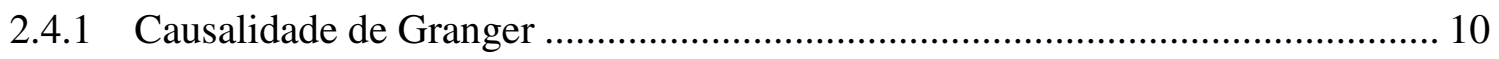

2.5 Variância de longo prazo ..................................................................................... 10

Capítulo 3 Séries de Memória Longa......................................................................... 12

3.1 Modelos auto-regressivo fracionariamente integrado e de médias móveis (ARFIMA) 13

3.2 Testes para Memória Longa ………………………………………………….... 14

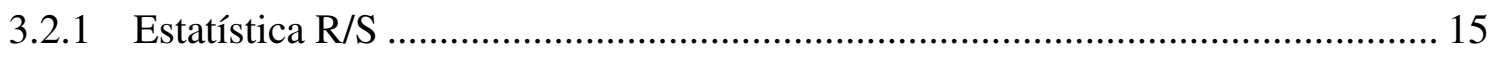




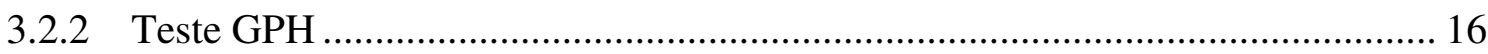

3.3 Estimadores para os Parâmetros de Memória Longa ............................................... 17

3.3.1 Estatística R/S ................................................................................ 17

3.3.2 Método do Periodograma ......................................................................... 18

3.3.3 Método de Whittle............................................................................................ 19

3.3.4 Estimador de Máxima Verossimilhança do Modelo ARFIMA ........................ 19

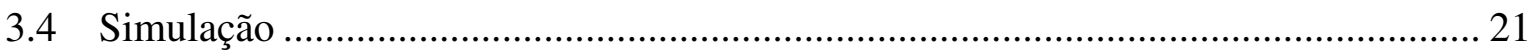

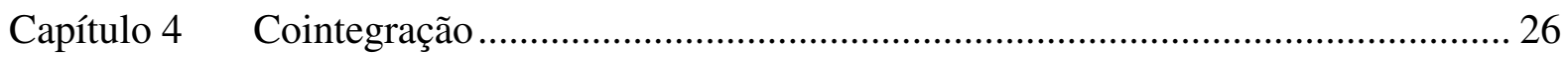

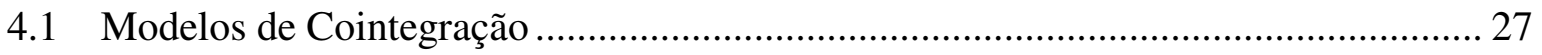

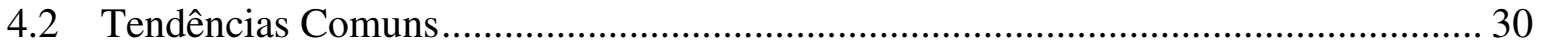

4.3 Modelo de Correção de Erros .............................................................................. 31

4.4 Testes para Cointegração .............................................................................. 33

4.4.1 Procedimento de Engle e Granger......................................................... 34

4.4.2 Regressão e modelo de correção de erros ....................................................... 35

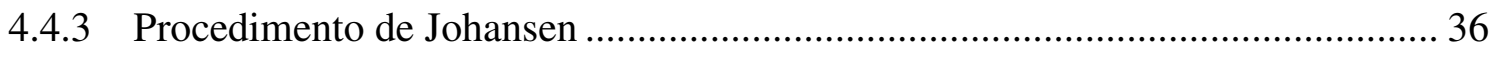

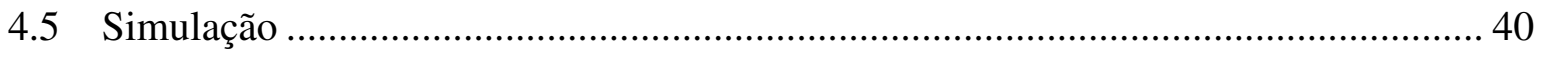

Capítulo 5 Cointegração Fracionária....................................................................... 45

5.1 Modelo de Correção de Erros ...................................................................... 47

5.2 Testes para Cointegração Fracionária............................................................... 48

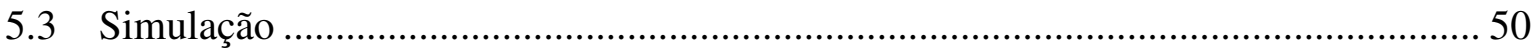

Capítulo 6 Aplicações em Séries Reais .................................................................... 55 
6.1 Estimação do Modelo de Cointegração Convencional ............................................ 56

6.2 Estimação do Modelo de Cointegração Fracionária................................................ 60

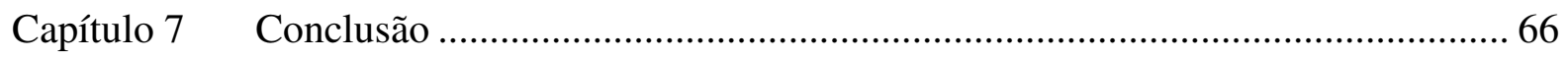

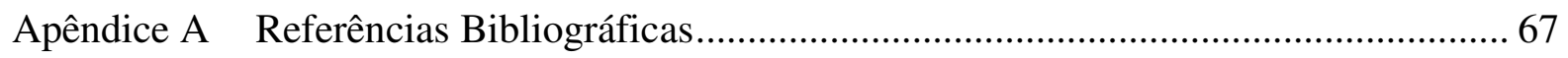

Apêndice B Comandos Splus ..................................................................................... 70 


\section{Capítulo 1 Introdução}

No mercado financeiro, grande parte das séries de preços de ativos são realizações de processos não estacionários, geralmente possuindo raízes unitárias, ou seja, são integradas $\mathrm{I}(d)$, $d \in \mathbb{N}$. Além disso, modelos econômicos demonstram diversas relações entre tais séries.

No entanto, a modelagem das relações entre séries não estacionárias por simples modelos de regressão ou pela utilização de modelos auto-regressivos vetoriais (VAR), que assumem que as séries sejam estacionárias, podem levar a conclusões erradas. Tais métodos quando aplicados a séries integradas apresentam resultados que podem não ser válidos, como o caso de regressão espúria apresentado por Granger e Newbold (1974).

Para se determinar a verdadeira relação entre estas séries foram criados os métodos de cointegração, como descrito por Engle e Granger (1987), que testam e modelam a relação entre séries não estacionárias, encontrando a relação estocástica de longo prazo entre elas. Estes métodos de cointegração convencional são bem conhecidos e utilizados.

No entanto, o fato de assumir valores inteiros para o parâmetro $d$ foi criticado e método alternativos propõem a utilização de processos fracionários $\mathrm{I}(d), d \in \mathbb{R}$, que possuem o mesmo problema de regressão espúria e aos quais não é possível a aplicação da análise de cointegração convencional.

Neste trabalho, serão apresentados os conceitos deste modelo de cointegração fracionária, bem como sua comparação com os métodos de cointegração convencionais, com foco principal na aplicação destes a séries reais.

No Capítulo 2, são apresentados alguns conceitos básicos que serão utilizados em capítulos posteriores.

No Capítulo 3, são apresentados conceitos de modelos de memória longa, inclusive testes para verificar a presença da persistência em séries e a estimação de parâmetros, cujo desempenho será avaliado através de simulações de diferentes modelos auto-regressivos fracionariamente integrados e de médias móveis (ARFIMA). 
A conceituação de cointegração é exposta no capítulo 4 em que são apresentados a presença de regressão espúria em séries não estacionárias, testes e estimação de parâmetros que serão testados pelas simulações de séries não estacionárias cointegradas e não cointegradas.

O restante deste trabalho está disposto da seguinte forma: testes e a modelagem de cointegração fracionária são observados no capítulo 5, bem como o procedimento de Davidson (2002) que utiliza a amostragem de bootstrap para realização dos testes; Aplicações a dados reais são apresentados no capítulo 6, em que as séries utilizadas são os preços de taxa de câmbio a vista e futuro negociados na $B M \& F$ e algumas considerações sobre os métodos são apresentadas no último capítulo, além de sugestões para trabalhos futuros. 


\section{Capítulo 2 Preliminares}

Este capítulo fornece, de forma resumida, o embasamento necessário para o desenvolvimento do tema proposto. Mais detalhes podem ser observados em Box, Jenkins e Reinsel (1994), Morettin e Toloi (2004), Morettin (2006) e Tsay (2005).

\subsection{Processos Estocásticos}

Um processo estocástico é um conjunto $Z=\{Z(t, \omega), t \in T, \omega \in \Omega\}$, tal que, $Z(t, \omega)$ é, para cada $t \in T$, uma variável aleatória contida num espaço de probabilidade $(\Omega, \Lambda, \mathrm{P})$ com $\omega \in \Omega$. Para cada $\omega \in \Omega$ obtém-se uma função de $t$, chamada de trajetória ou série temporal.

Um série temporal $Z(t, \omega)$, ou $z$, é chamada estritamente estacionária se a distribuição conjunta de $\left(z_{t 1}, z_{t 2}, \cdots z_{t k}\right)$, em que $k$ é um inteiro positivo qualquer, é idêntica à distribuição conjunta de $\left(z_{t 1+x}, z_{t 2+x}, \cdots z_{t k+x}\right)$ para todo $x$, ou seja, a distribuição de $\left(z_{t 1}, z_{t 2}, \cdots z_{t k}\right)$ deve ser a mesma para qualquer janela de tempo. Por ser uma condição difícil de se verificar empiricamente, geralmente é utilizado o conceito de estacionariedade fraca, também conhecido como estacionariedade de segunda ordem.

Uma série $Z_{t}$ é fracamente estacionária se:

- $E\left(z_{t}\right)=\mu$ é constante para todo $t$

- $\operatorname{cov}\left(z_{t}, z_{t+k}\right)=E\left[\left(z_{t}-\mu\right)\left(z_{t+k}-\mu\right)\right]=\gamma_{k}$ depende somente de $k$, para todo $t$ e qualquer $k$;

- $E\left(z_{t}^{2}\right)<\infty$ para qualquer $t$

A função de autocovariância $\gamma_{k}$ satisfaz as seguintes propriedades:

- $\gamma_{0}=E\left[\left(z_{t}-\mu\right)^{2}\right]>0$;

- $\gamma_{-k}=\gamma_{k}$

- $\gamma_{0} \geq\left|\gamma_{k}\right|$

- $\quad \gamma_{k}$ é não negativa definida;

A função de autocorrelação (FAC) do processo é definida por $\rho_{k}=\gamma_{k} / \gamma_{0}, k \in Z$. 
A FAC amostral de defasagem $k$ pode ser obtida por

$$
\hat{\rho}_{k}=\hat{\gamma}_{k} / \hat{\gamma}_{0}
$$

sendo a autocovariância amostral definida por

$$
\hat{\gamma}_{k}=\frac{1}{T} \sum_{t=k+1}^{T}\left(z_{t}-\bar{z}\right)\left(z_{t-k}-\bar{z}\right),
$$

e a média amostral $\bar{z}=\frac{1}{T} \sum_{t=1}^{T} Z_{t}$.

Quando estes valores amostrais, chamados de momentos amostrais, convergem em

probabilidade para os momentos populacionais da série $z_{t}\left(\hat{\gamma}_{k} \stackrel{p}{\rightarrow} \gamma_{k}, \hat{\rho}_{k} \stackrel{p}{\rightarrow} \rho_{k}, \bar{z} \stackrel{p}{\rightarrow} \mu\right)$ pode-se afirmar que a série é ergódica.

\subsubsection{Movimento Browniano}

Movimento Browniano Padrão, também conhecido como processo de Wiener, é um processo contínuo $\mathrm{W}=\{\mathrm{W}(\mathrm{t}), \mathrm{t} \geq 0\}$ tal que:

- $\mathrm{W}(0)=0$;

- As variáveis aleatórias (v.a.), $\mathrm{W}\left(\mathrm{t}_{\mathrm{k}}\right)-\mathrm{W}\left(\mathrm{t}_{\mathrm{k}-1}\right) \mathrm{e} \mathrm{W}\left(\mathrm{t}_{\mathrm{j}}\right)-\mathrm{W}\left(\mathrm{t}_{\mathrm{j}-1}\right)$ são independentes para quaisquer $\mathrm{k}$ e $\mathrm{j}$;

- As v.a. $\mathrm{W}(\mathrm{t})-\mathrm{W}(\mathrm{s})$ e $\mathrm{W}(\mathrm{t}+\tau)-\mathrm{W}(\mathrm{s}+\tau)$ possuem a mesma distribuição para quaisquer $t, s$ e $\tau$;

- $\mathrm{W}(\mathrm{t}) \sim N(0, \mathrm{t})$;

- As trajetórias de W(t) são contínuas com probabilidade um.

O Movimento Browniano Padrão possui $E[\mathrm{~W}(\mathrm{t})]=0$ e $\gamma_{k}=\gamma(t, t+k)=\min (t, t+k)$.

\subsection{Modelo ARMA}

Uma série temporal $z_{t}$ é chamada linear quando pode ser escrita como: 


$$
z_{t}=\mu+\sum_{k=0}^{\infty} \psi_{\mathrm{k}} \mathrm{a}_{\mathrm{t}-\mathrm{k}}
$$

com $\Psi_{0}=1$, e $\left\{a_{t}\right\}$ é uma sequência de variáveis aleatórias independentes e identicamente distribuidas (I.I.D.) com média 0 e variância $\sigma^{2}$, i.e., $\mathrm{a}_{\mathrm{t}} \sim \mathrm{RB}\left(0, \sigma^{2}\right)$ sendo $\mathrm{RB}$ a sigla para ruído branco.

\subsubsection{Modelo auto-regressivo (AR)}

O modelo auto-regressivo de ordem $\mathrm{p}(\mathrm{AR}(\mathrm{p}))$ possui a seguinte forma (admitindo-se $\mu=0$ ):

$$
z_{t}=\phi_{1} z_{t-1}+\phi_{2} z_{t-2}+\cdots+\phi_{\mathrm{p}} z_{t-p}+a_{t}
$$

que pode ser escrito na notação de operadores

$$
\phi(\mathrm{B}) z_{t}=a_{t}
$$

em que B é o operador de defasagem $\left(B^{k} z_{t}=z_{t-k}\right)$ e $\phi(\mathrm{B})=1-\phi_{1} \mathrm{~B}-\phi_{2} \mathrm{~B}^{2}-\cdots \phi_{\mathrm{p}} \mathrm{B}^{\mathrm{p}}$.

Este processo é considerado invertível, sendo considerado estacionário e ergódico no caso das raízes da equação característica $\phi(B)=0$ estejam fora do círculo unitário.

Sua função de autocorrelação de ordem k é dada por

$$
\rho_{\mathrm{k}}=\phi_{1} \rho_{\mathrm{k}-1}+\phi_{2} \rho_{\mathrm{k}-2}+\cdots+\phi_{\mathrm{p}} \rho_{\mathrm{k}-\mathrm{p}}
$$

No caso de processos auto-regressivos, a função de autocorrelação é definida por uma mistura de polinômios, exponenciais e senóides.

A função de autocorrelação parcial $(\mathrm{FACP}) \phi_{\mathrm{kk}}$ de uma série representa a correlação parcial entre as variáveis $z_{t}$ e $z_{t-k}$ eliminada a influência de $z_{t-1}, z_{t-2}, \ldots, z_{t-k+1}$. Ela pode ser obtida pela estimação de modelos AR de diversas ordens, ou seja,

$$
\begin{aligned}
& z_{t}=\phi_{11} z_{t-1}+a_{1 t} \\
& z_{t}=\phi_{21} z_{t-1}+\phi_{22} z_{t-2}+a_{2 t} \\
& \vdots
\end{aligned}
$$




$$
z_{t}=\phi_{\mathrm{p} 1} z_{t-1}+\phi_{\mathrm{p} 2} z_{t-2}+\cdots+\phi_{\mathrm{pp}} z_{t-p}+a_{p t}
$$

em que os parâmetros $\phi_{\mathrm{kk}}$ para $\mathrm{k}=1,2, \ldots, \mathrm{p}$ são os k-ésimos coeficientes de autocorrelação parcial.

Para processos auto-regressivos de ordem p, todos os coeficientes de autocorrelação parcial de ordem $\mathrm{k}$ são nulos para $\mathrm{k}>p$.

\subsubsection{Modelo de médias móveis (MA)}

O modelo de médias móveis de ordem q MA(q) possui a seguinte forma:

$$
z_{t}=a_{t}+\theta_{1} a_{t-1}+\theta_{2} a_{t-2}+\cdots+\theta_{\mathrm{q}} a_{t-q}
$$

Utilizando a notação de operadores $\theta(B)=1+\theta_{1} B+\theta_{2} B^{2}+\cdots+\theta_{q} B^{q}$, tem-se

$$
z_{t}=\theta(\mathrm{B}) a_{t}
$$

Estes modelos são estacionários e são considerados invertíveis desde que a equação característica $\theta(B)=0$ possua todas as raízes fora do círculo unitário.

A FAC do processo MA(q) é dada por

$$
\rho_{\mathrm{k}}=\frac{\theta_{\mathrm{k}}+\theta_{\mathrm{k}+1} \theta_{1}+\theta_{\mathrm{k}+2} \theta_{2}+\cdots+\theta_{\mathrm{q}} \theta_{\mathrm{q}-\mathrm{k}}}{1+\theta_{1}^{2}+\theta_{2}^{2}+\theta_{\mathrm{q}}^{2}}
$$

para $\mathrm{k} \leq \mathrm{q}$ e é nula para $\mathrm{k}>q$

Para séries MA(q), todos os coeficientes da FAC são nulos, com excessão do q-ésimo coeficiente, enquanto a FACP deste processo apresenta decaimento exponencial ou senoidal.

\subsubsection{Modelo auto-regressivo e de médias móveis}

O modelo auto-regressivo de ordem p e de médias móveis de ordem q (ARMA(p,q)) é dado por:

$$
z_{t}=\phi_{1} z_{t-1}+\phi_{2} z_{t-2}+\cdots+\phi_{\mathrm{p}} z_{t-p}+a_{t}+\theta_{1} a_{t-1}+\theta_{2} a_{t-2}+\cdots+\theta_{\mathrm{q}} a_{t-q}
$$

Na notação de operadores, tem-se: 


$$
\phi(\mathrm{B}) z_{t}=\theta(\mathrm{B}) a_{t} .
$$

Este modelo é considerado estacionário caso as raízes da equação $\phi(B)=0$ estiverem fora do círculo unitário e invertível caso as raízes da equação $\theta(B)=0$ estiverem fora do círculo unitário.

\subsection{Séries Temporais não Estacionárias}

Os modelos apresentados até agora são aplicáveis a séries estacionárias, que, portanto, evoluem em torno de uma média constante. Em geral, a presença de não estacionariedade em uma série deve-se ao fato de não existir um nível fixo para a evolução da mesma.

Séries não estacionárias $\left(\mathrm{x}_{t}\right)$, cuja a série formada por $\mathrm{d}$ diferenças da mesma (caso $\mathrm{d}=1$, $\left.z_{t}=\Delta^{d} x_{t}=(1-\mathrm{B})^{d} x_{t}=x_{t}-x_{t-1}\right)$ é estácionária, são chamadas não estacionárias homogêneas, portadoras de raízes unitárias ou integradas de ordem d e denotadas por I(d).

\subsubsection{Modelo ARIMA}

Se $z_{t}=\Delta^{d} x_{t}$ for estacionária, um modelo ARMA(p,q) pode ser ajustado para a série $z_{t}$ :

$$
\phi(\mathrm{B}) z_{t}=\theta(\mathrm{B}) a_{t} \Rightarrow \phi(\mathrm{B}) \Delta^{d} x_{t}=\theta(\mathrm{B}) a_{t}
$$

modelo nomeado como auto-regressivo, integrado e de médias móveis ARIMA(p,d,q).

\subsubsection{Teste de raízes unitárias}

Dada uma série $z_{t}$, com $t=1,2, \ldots, T$ cujo comportamento segue o modelo

$$
\begin{gathered}
z_{t}=\phi z_{t-1}+a_{t}, \quad a_{\mathrm{t}} \sim \operatorname{RBN}\left(0, \sigma_{a}^{2}\right), \text { ou } \\
\Delta z_{t}=\phi^{*} z_{t-1}+a_{t}, \quad \phi^{*}=\phi-1 .
\end{gathered}
$$

Por mínimos quadrados são calculados

$$
\widehat{\phi}^{*}=\frac{\sum_{\mathrm{t}=1}^{\mathrm{T}} z_{t-1} a_{t}}{\sum_{\mathrm{t}=1}^{\mathrm{T}} \mathrm{z}_{\mathrm{t}-1}^{2}} e \hat{\sigma}_{a}^{2}=\frac{\sum_{\mathrm{t}=2}^{\mathrm{T}}\left(\Delta z_{t}-\hat{\phi}^{*} z_{t-1}\right)^{2}}{T-2} .
$$

O teste se baseia nas seguintes hipóteses 
$\mathrm{H}_{0}^{*}: \phi^{*}=0$, existência de uma raiz unitária,

$\mathrm{H}_{1}^{*}: \phi^{*}<0$, não existência de raiz unitária.

Fazendo-se uso da estatística

$$
\hat{\tau}=\frac{\widehat{\phi}^{*}}{\widehat{e p}\left(\widehat{\phi}^{*}\right)}, \quad \widehat{e p}(\widehat{\phi})=\frac{\hat{\sigma}_{a}}{\sqrt{\sum_{\mathrm{t}=1}^{\mathrm{T}} \mathrm{z}_{\mathrm{t}-1}^{2}}} \Rightarrow \hat{\tau}=\frac{\sum_{\mathrm{t}=1}^{\mathrm{T}} z_{t-1} a_{t}}{\hat{\sigma}_{a} \sqrt{\sum_{\mathrm{t}=1}^{\mathrm{T}} \mathrm{z}_{\mathrm{t}-1}^{2}}}
$$

Caso $\left\{a_{t}\right\}$ for uma série de ruído branco com momentos finitos, a estatística, também conhecida como estatística de Dickey-Fuller, convergirá para uma função do movimento browniano padrão. Mais detalhes em Dickey e Fuller (1979).

O modelo pode ser alterado para $\mathrm{AR}(\mathrm{p})$

$$
\Delta z_{t}=\theta+\beta z_{t-1}+\sum_{i=1}^{p-1} \phi_{i} \Delta z_{t-i}+a_{t}
$$

sendo utilizada a estatística

$$
\operatorname{ADF}=\frac{\widehat{\beta}}{\widehat{e p}(\widehat{\beta})}
$$

e testado $H_{0}: \beta=0$ vs $H_{1}: \beta<0$. Teste este conhecido como teste Dickey Fuller Aumentado (Augmented Dickey Fuller - ADF) sendo as estatísticas tabuladas.

\subsection{Modelo VAR}

O modelo auto regressivo vetorial (VAR) é um dos mais utilizados, flexível e simples para análise de séries temporais multivariadas, sendo uma extensão natural do modelo autoregressivo univariado. Admitindo-se o vetor de variáveis no tempo $\mathrm{Z}_{\mathrm{t}}=\left(\mathrm{z}_{1 \mathrm{t}}, \mathrm{z}_{2 \mathrm{t}}, \ldots, \mathrm{z}_{\mathrm{nt}}\right)^{\prime}$, o modelo VAR(p) possui a seguinte forma:

$$
Z_{t}=\mathrm{c}+\Pi_{1} Z_{t-1}+\Pi_{2} Z_{t-2}+\cdots+\Pi_{\mathrm{p}} Z_{t-p}+\varepsilon_{\mathrm{t}}, \quad t=1, \ldots, T
$$


em que $\Pi_{i}$ são matrizes $(n \times n)$ de coeficientes e $\varepsilon_{t}$ é um processo vetorial ruído branco com média nula $(n \times 1)$ e matriz de covariância fixa $\Sigma$.

O modelo também pode ser escrito na notação de operadores

$$
\Pi(\mathrm{B}) Z_{t}=\mathrm{c}+\varepsilon_{\mathrm{t}}
$$

em que $\Pi(B)=I_{n}-\Pi_{1} B-\Pi_{2} B^{2}-\cdots-\Pi_{p} B^{p}$ e $I_{n}$ a matriz identidade $(n \times n)$. Sendo estacionário quando as raízes da equação

$$
\operatorname{det}\left(I_{n}-\Pi_{1} z-\Pi_{2} z^{2}-\cdots-\Pi_{p} z^{p}\right)=0
$$

encontrarem-se fora do círculo unitário. Admitindo-se estacionariedade a média do processo será dada por

$$
\mu=\left(\mathrm{I}_{\mathrm{n}}-\Pi_{1}-\Pi_{2}-\cdots-\Pi_{\mathrm{p}}\right)^{-1} \mathrm{c}
$$

e o modelo ajustado será, portanto,

$$
Z_{t}-\mu=\Pi_{1}\left(Z_{t-1}-\mu\right)+\Pi_{2}\left(Z_{t-2}-\mu\right)+\cdots+\Pi_{\mathrm{p}}\left(Z_{t-p}-\mu\right)+\varepsilon_{\mathrm{t}}
$$

A identificação da ordem do modelo pode ser efetuada através de ajustes sequênciais de modelos VAR de ordens $1,2, \ldots, k$ e testar a significância dos coeficientes:

$$
\mathrm{H}_{0}: \Pi_{\mathrm{k}}^{(\mathrm{k})}=0 \text { vs } \mathrm{H}_{1}: \Pi_{\mathrm{k}}^{(\mathrm{k})} \neq 0
$$

Testa-se também a covariância dos resíduos dos modelos ajustados por razão de verossimilhança. Tem-se para a k-ésima equação ajustada os seguintes resíduos

$$
\widehat{\varepsilon}_{\mathrm{t}}^{(\mathrm{k})}=Z_{t}-\hat{\mu}-\widehat{\Pi}_{1}^{(\mathrm{k})}\left(Z_{t-1}-\hat{\mu}\right)+\widehat{\Pi}_{2}^{(\mathrm{k})}\left(Z_{t-2}-\hat{\mu}\right)+\cdots+\widehat{\Pi}_{\mathrm{k}}^{(\mathrm{k})}\left(Z_{t-k}-\hat{\mu}\right)
$$

cuja matriz de covariância estimada é dada por

$$
\hat{\Sigma}^{(\mathrm{k})}=\frac{1}{\mathrm{~T}-\mathrm{k}} \sum_{t=k+1}^{T} \widehat{\varepsilon}_{\mathrm{t}}^{(\mathrm{k})}\left(\widehat{\varepsilon}_{\mathrm{t}}^{(\mathrm{k})}\right)^{\prime}
$$

e a razão de verossimilhanças para o teste 


$$
\mathrm{RV}(\mathrm{k})=(\mathrm{T}-\mathrm{k}) \ln \frac{\left|\hat{\Sigma}^{(\mathrm{k}-1)}\right|}{\left|\hat{\Sigma}^{(\mathrm{k})}\right|},
$$

que possui distribuição qui-quadrado com $n^{2}$ graus de liberdade, $\chi^{2}\left(n^{2}\right)$.

\subsubsection{Causalidade de Granger}

Granger (1969) define como causalidade o fato de se poder prever de forma mais eficiente o valor de uma variável Y qualquer utilizando valores passados de uma variável X. Neste caso, diz-se que a variável $\mathrm{X}$ granger-causa $\mathrm{Y}$.

Admitindo-se $\left\{A_{t}, t=0, \pm 1, \pm 2, \ldots\right\}$ o conjunto de informação relevante até o instante $t$, contendo $X_{t}$ e $Y_{t}, P_{t}(Y \mid B)$ o preditor de mínimo erro quadrático médio de $Y$ utilizando-se o conjunto de informações B e $\sigma^{2}(Y \mid B)$ o correspondente erro quadrático médio do preditor. Pode-se dizer que:

- $X_{t} \rightarrow Y_{t}: X_{t}$ causa $Y_{t}$ (causalidade unidirecional) se $\sigma^{2}\left(Y_{t} \mid A_{t-1}\right)<\sigma^{2}\left(Y_{t} \mid A_{t-1}-\right.$ $\left.X_{t-1}-X_{t-2}-X_{t-3}-\cdots\right)$, ou seja, $Y_{t}$ pode ser melhor prevista se tivermos toda a informação passada de $X_{t}$ e $Y_{t}$. Neste caso pode-se dizer, também, que $X_{t}$ é exogena ou antecedente a $Y_{t}$;

- $X_{t} \Rightarrow Y_{t}: X_{t}$ causa instantaneamente $Y_{t}$ se $\sigma^{2}\left(Y_{t} \mid A_{t-1}, X_{t}\right)<\sigma^{2}\left(Y_{t} \mid A_{t-1}\right)$, ou seja, o valor atual de $Y_{t}$ pode ser melhor previsto se o valor presente de $X_{t}$ for conhecido;

- $\quad$ Existe feedback $\left(X_{t} \leftrightarrow Y_{t}\right)$ se $X_{t}$ causa $Y_{t}$ e $Y_{t}$ causa $X_{t}$;

\subsection{Variância de longo prazo}

Seja $z_{t}$ uma série estacionária e ergódica. Pelo teorema central do limite

$$
\sqrt{T}(\overline{\mathrm{z}}-\mu) \stackrel{d}{\rightarrow} N\left(0, \sum_{k=-\infty}^{\infty} \gamma_{k}\right)
$$

em que $\mu$ é a média populacional, $\bar{z}$ é a média amostral, $T$ é o tamanho da amostra e $\gamma_{k}$ é a autocovariância de termos defasados por $k$ períodos. Assintoticamente tem-se 


$$
\overline{\mathrm{z}} \sim N\left(\mu, \frac{1}{T} \sum_{k=-\infty}^{\infty} \gamma_{k}\right)
$$

A variância de longo prazo (long run variance - lrv) é dada pela multiplicação de $T$ pela variância assintótica da média amostral, ou seja,

$$
\operatorname{lrv}\left(z_{t}\right)=T \cdot \frac{1}{T} \sum_{k=-\infty}^{\infty} \gamma_{k}=\sum_{k=-\infty}^{\infty} \gamma_{k} .
$$

Um popular estimador para a variância de longo prazo foi apresentado por Newey e West (1987):

$$
\widehat{\operatorname{rv}}\left(z_{t}\right)=\hat{\gamma}_{0}+2 \sum_{k=1}^{M_{T}} \omega_{k, T} \hat{\gamma}_{k}
$$

em que $\omega_{k, T}$ são pesos cuja soma é 1 e $M_{T}$ é um parâmetro de truncamento do lag que satisfaz $M_{T}=O\left(T^{1 / 3}\right)$.

Para processos lineares em geral, os autores sugerem a utilização de pesos da forma $\omega_{k, T}=$ $1-\frac{j}{M_{T}+1}$, com $M_{T}$ sendo a parte inteira de $M_{T}=4(T / 100)^{2 / 9}$. 


\section{Capítulo 3 Séries de Memória Longa}

Devido ao decaimento exponencial para zero da função de autocorrelação de processos $\operatorname{ARMA}(p, q)$, estes são conhecidos como processos de memória curta ou alta frequência. Pode-se mostrar que processos não estacionários com raízes unitárias apresentam FAC convergente para um. Existem ainda processos em que a FAC decai lentamente para zero, ou seja, observações distantes possuem significante dependência, indicando persistência nos valores de autocorrelação. Tais séries são conhecidas como processos de memória longa ou processos fracionários.

A figura abaixo apresenta um exemplo de série temporal de memória longa e sua FAC. Podese notar o lento decaimento de sua função de autocorrelação que possui valores significantes para defasagens maiores que 150 .
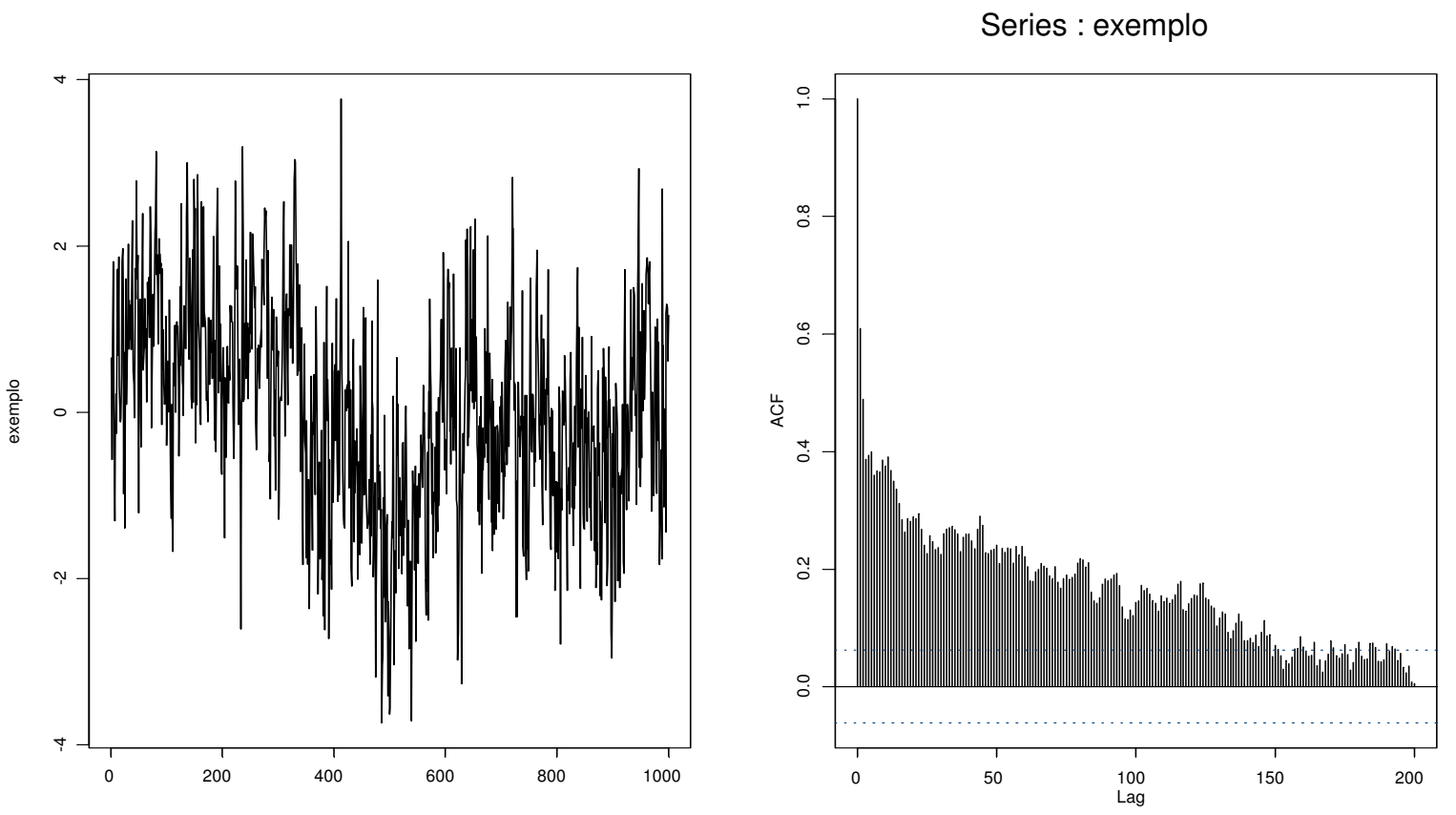

Figura 3.1 - Exemplo de série de memória longa e sua função de auto correlação

A FAC de um processo fracionariamente decresce de acordo com a seguinte equação

$$
\rho_{k} \sim C k^{-\alpha}, k \rightarrow \infty
$$


para C $>0$ e $0<\alpha<1$.

Outra importante função para análise de processos estacionários é a densidade espectral, definida por

$$
f(\omega)=\frac{1}{2 \pi} \sum_{k=-\infty}^{\infty} \rho_{k} e^{i k \omega},
$$

em que $\omega$ é a frequência de Fourier.

Por (3.1) e (3.2) pode-se concluir que a função densidade espectral de um processo com memória longa é dada por

$$
f(\omega) \rightarrow C_{f} \omega^{\alpha-1} \text { quando } \omega \rightarrow 0,
$$

sendo $C_{f}$ uma constante positiva. Na prática, ao invés da variável $\alpha$, é utilizado o coeficiente de Hurst - $H$ (Hurst, 1951) para mensurar a memória longa de um processo.

$$
H=1-\alpha / 2 \in(0,5 ; 1) .
$$

Quanto maior o valor de $H$, mais lento é o decaimento da FAC do processo, indicando, portanto, a presença de memória mais longa.

\subsection{Modelos auto-regressivo fracionariamente integrado e de médias móveis (ARFIMA)}

Um processo auto-regressivo fracionariamente integrado e de médias móveis $\operatorname{ARFIMA}(\mathrm{p}, d, \mathrm{q})$, sendo $d=H-1 / 2$, é dado por

$$
\boldsymbol{\phi}(\mathrm{B})(\mathbf{1}-B)^{d} z_{t}=\boldsymbol{\theta}(\mathrm{B}) \boldsymbol{a}_{t},
$$

em que o operador de diferença fracionária é definido da seguinte maneira:

$$
(1-B)^{d}=\sum_{k=0}^{\infty}\left(\begin{array}{l}
d \\
k
\end{array}\right)(-B)^{k}=1-d B+\frac{1}{2 !} d(d-1) B^{2}-\frac{1}{3 !} d(d-1)(d-2) B^{3}+\cdots,
$$

para qualquer real $d>-1$. 
Este processo se ajusta a séries com comportamento de memória longa através do parâmetro de diferenciação $d$, enquanto os parâmetros $\phi$ e $\theta$ explicam a estrutura de correlação de curto prazo, de forma que

- O modelo será estacionário caso $d<\frac{1}{2}$ e as raízes de $\phi(\mathrm{B})=0$ estiverem fora do círculo unitário;

- Será invertível caso $d>-\frac{1}{2}$ e as raízes de $\theta(B)=0$ estiverem fora do círculo unitário.

O Caso mais simples, $\operatorname{ARFIMA}(0, d, 0)$ conhecido como ruído branco fracionário, possui a seguinte forma:

$$
(1-B)^{d} z_{t}=a_{t}
$$

- Quando $-\frac{1}{2}<d<\frac{1}{2}$, o processo é estacionário e invertível;

- $\quad$ Para $-\frac{1}{2}<d<\frac{1}{2}$

- A função espectral é dada por: $f(\omega)=\left\{\begin{array}{c}\frac{\sigma_{\mathrm{t}}^{2}}{2 \pi}\left(2 \operatorname{sen}\left(\frac{\omega}{2}\right)\right)^{-2 d}, 0<\omega \leq \pi \\ \omega^{-2 d}, \omega \rightarrow 0\end{array}\right.$

○ A FAC é dada por: $\rho_{\mathrm{k}}=\frac{(-d) !(\mathrm{k}+d-1) !}{(d-1) !(\mathrm{k}-d) !}=\prod_{0 \leq \mathrm{x}<k} \frac{\mathrm{x}-1+d}{\mathrm{x}-d}, \mathrm{k}=1,2, \ldots$

○ A FACP representada por: $\phi_{k k}=\frac{d}{k-d}, k=1,2, \ldots$

○ A variância definida por: $\rho_{\mathrm{k}}=\frac{(-2 d) !}{(-d) !^{2}}$

\subsection{Testes para Memória Longa}

Vários testes foram formulados para determinar a existência de memória longa em séries temporais. Serão expostos a estatística R/S e o teste GPH. É importante ressaltar, contudo, que a definição de memória longa é especificada pelo comportamento assintótico da FAC quando $k \rightarrow \infty$ ou pela densidade espectral quando $\omega \rightarrow 0$. 


\subsubsection{Estatística $\mathbf{R} / \mathbf{S}$}

$\mathrm{O}$ teste que utiliza a estatística R/S (rescaled range ou range over standard deviation) é o mais conhecido para verificar a dependência de longo prazo ou memória longa em séries temporais.

Proposto por Hurst (1951), a estatística R/S consiste na mudança de escala da amplitude da soma parcial dos desvios com relação à média de uma série temporal pelo desvio padrão. Considerando a série $z_{t}$, para $\mathrm{t}=1, \ldots, T$, a estatística R/S é definida por:

$$
Q_{T}=\frac{1}{S_{T}}\left[\max _{1 \leq \mathrm{k} \leq \mathrm{T}} \sum_{\mathrm{t}=1}^{\mathrm{k}}\left(z_{t}-\overline{\mathrm{z}}\right)-\min _{1 \leq \mathrm{k} \leq \mathrm{T}} \sum_{\mathrm{t}=1}^{\mathrm{k}}\left(z_{t}-\overline{\mathrm{z}}\right)\right],
$$

em que $\overline{\mathrm{z}}=1 / \mathrm{T} \sum_{\mathrm{t}=1}^{\mathrm{T}} z_{t}$ é a média amostral e $s_{T}=\sqrt{1 / \mathrm{T} \sum_{\mathrm{t}=1}^{\mathrm{T}}\left(z_{t}-\overline{\mathrm{z}}\right)^{2}}$ é o desvio padrão amostral. Se os $z_{t}$ 's forem v.a.normais i.i.d., então $\frac{1}{\sqrt{T}} Q_{T}$ converge fracamente para uma v.a. que está no domínio de atração de uma ponte browniana no intervalo unitário, cujos quantis são fornecidos por Lo (1991).

Lo (1991) também indica que a estatística R/S não é robusta para a dependência de memória curta e propõe a seguinte alteração

$$
\tilde{Q}_{T}=\frac{1}{\hat{\sigma}_{T}(\mathrm{q})}\left[\max _{1 \leq \mathrm{k} \leq \mathrm{T}} \sum_{\mathrm{t}=1}^{\mathrm{k}}\left(z_{t}-\overline{\mathrm{z}}\right)-\min _{1 \leq \mathrm{k} \leq \mathrm{T}} \sum_{\mathrm{t}=1}^{\mathrm{k}}\left(z_{t}-\overline{\mathrm{z}}\right)\right] \text {, }
$$

em que, $\hat{\sigma}_{T}(q)$ é a raiz quadrada do estimador da variância de longo prazo de Newey-West, com largura de faixa q, dado por

$$
\hat{\sigma}_{T}^{2}(q)=s_{t}^{2}\left(1+\frac{2}{T} \sum_{j=1}^{q}\left(1-\frac{j}{q+1}\right) \hat{\rho}_{j}\right)
$$

sendo, $\hat{\rho}_{\mathrm{j}}$ as autocorrelações amostrais e $\mathrm{q}<T$. 


\subsubsection{Teste GPH}

Baseados no processo fracionariamente integrado:

$$
(1-B)^{d} z_{t}=a_{t}
$$

em que $a_{t} \sim R B$, Geweke e Porter-Hudak (1983) propuseram um teste semiparamétrico para memória longa. A função densidade espectral do processo é dada por:

$$
f(\omega)=\left[4 \operatorname{sen}^{2}\left(\frac{\omega}{2}\right)\right]^{-\mathrm{d}} f_{a}(\omega)
$$

em que $\omega$ é a frequência de Fourier e $f_{a}(\omega)$ é a função densidade espectral de $a_{t}$. O parâmetro de diferença fracionária $d$ pode ser obtido pela regressão

$$
\ln \left[f\left(\omega_{\mathrm{j}}\right)\right]=\beta-\mathrm{d} \ln \left[4 \operatorname{sen}^{2}\left(\frac{\omega_{\mathrm{j}}}{2}\right)\right]+e_{j}
$$

para $\mathrm{j}=1,2, \ldots, \mathrm{n}_{\mathrm{f}}(\mathrm{T})$. Geweke e Porter-Hudak (1983) mostraram que usando o periodograma como estimativa de $f\left(\omega_{\mathrm{j}}\right)$, a estimativa $\hat{d}$ de mínimos quadrados da regressão acima, possui distribuição normal para grandes amostras se $\mathrm{n}_{\mathrm{f}}(\mathrm{T})=\mathrm{T}^{\alpha} \operatorname{com} 0<\alpha<1$ :

$$
\hat{d} \sim N\left(d, \frac{\pi^{2}}{6 \sum_{\mathrm{j}=1}^{\mathrm{n}_{\mathrm{f}}}\left(\mathrm{U}_{\mathrm{j}}-\overline{\mathrm{U}}\right)}\right)
$$

em que $U_{j}=\ln \left[4 \operatorname{sen}^{2}\left(\frac{\omega_{j}}{2}\right)\right]$ e $\bar{U}$ a média amostral de $U_{j}, \operatorname{com} \mathrm{j}=1,2, \ldots, \mathrm{n}_{\mathrm{f}}$. Sob a hipótese nula de não existência de memória longa $(d=0)$, a estatística abaixo possui distribuição assintótica normal padrão.

$$
\mathrm{t}_{d=0}=\hat{d}\left(\frac{\pi^{2}}{6 \sum_{\mathrm{j}=1}^{\mathrm{n}_{\mathrm{f}}}\left(\mathrm{U}_{\mathrm{j}}-\overline{\mathrm{U}}\right)}\right)^{-1 / 2} .
$$




\subsection{Estimadores para os Parâmetros de Memória Longa}

Nas seções anteriores foram introduzidos dois testes da presença de memória longa em séries temporais: Estatística R/S e GPH. Esta seção apresentará como se obter estimativas para o parâmetro de memória longa $H$ ou $d$. No teste GPH, a estimativa de $d$ é diretamente obtida.

Será observado que a Estatística R/S também pode ser utilizada com o propósito de se encontrar estimativas para o parâmetro de memória longa, além de outros dois métodos baseados na análise do periodograma.

Outro método apresentado nesta seção será aquele gerado pela estimação do modelos $\operatorname{ARFIMA}(\mathrm{p}, d, \mathrm{q})$.

\subsubsection{Estatística R/S}

Madelbrot (1975), apresentou em seu trabalho que se uma série possui memória longa a estatística R/S converge para uma v.a. a uma taxa $T^{H}$, em que $H$ é o coeficiente de Hurst, enquanto séries que não possuem memória longa convergem a taxas $T^{1 / 2}$. Com isso, o gráfico em escala logarítmica da estatística R/S pelo tamanho da amostra de uma série temporal sem memória longa deve se distribuir em torno de uma linha reta com inclinação igual a $1 / 2 \mathrm{e}$, para o caso de uma série com memória longa, este gráfico se distribui ao redor de uma reta com coeficiente de inclinação $H>1 / 2$.

O procedimento para estimar o parâmetro $H$ parte da primeira estimativa da estatística $\mathrm{R} / \mathrm{S}$ utilizando-se $k_{1}$ observações consecutivas. Em seguida, eleva-se o tamanho da amostra por um fator $f$, ou seja, o número de observações será $k_{i}=f k_{i-1}$ para $i=2,3, \ldots$. Note que para calcular a estatística R/S com $k_{i}$ observações consecutivas pode-se dividir a amostra em $T / k_{i}$ blocos e obter $T / k_{i}$ valores diferentes. A inclinação da reta ajustada no gráfico gera a estimativa de $H$.

A Figura 3.2 demonstra a distribuição das estatísticas R/S calculadas para diferentes amostras da série exemplo apresentadas no início do capítulo. A linha sólida representa a reta ajustada e a linha pontilhada representa o caso de inexistência de memória longa. Pode-se observar que, 
como a reta ajustada está distante da linha pontilhada, há a presença de memória longa na série exemplo.

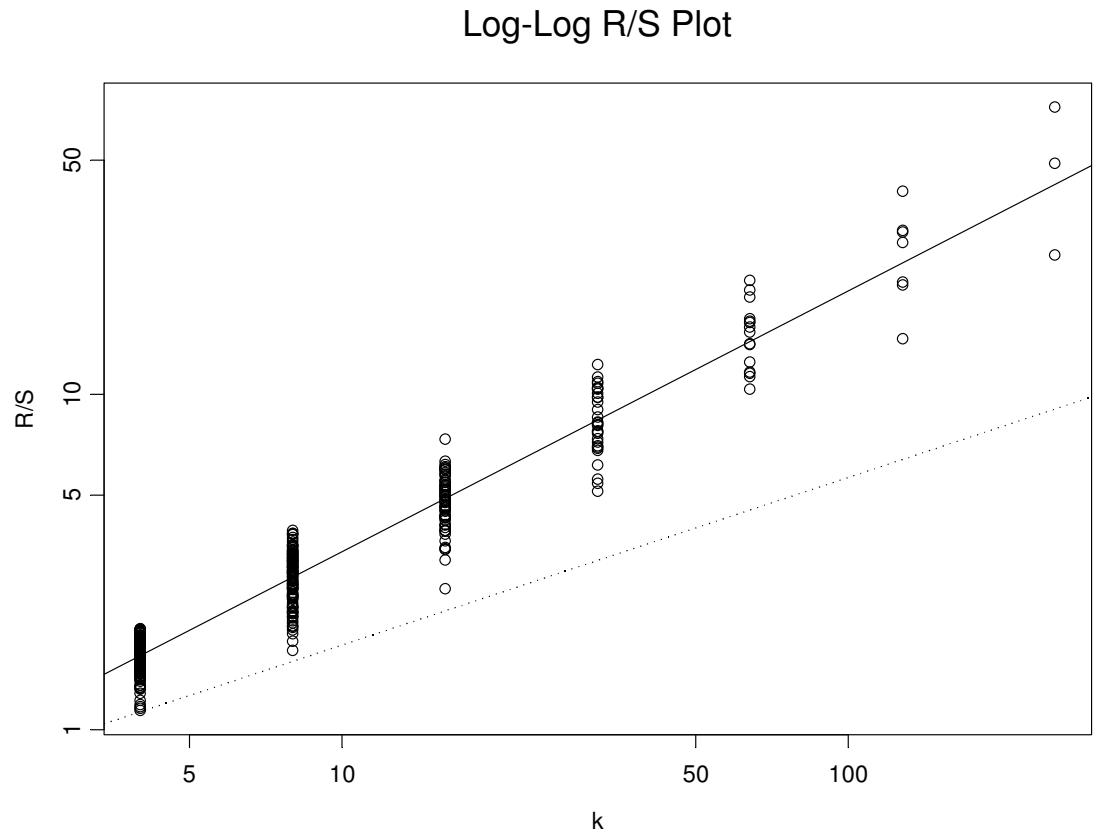

Figura 3.2 - Gráfico da Estatística R/S

Uma alternativa para se obter estimativas mais robustas do parâmetro de memória longa é utilizar o procedimento Least Absolute Deviation (LAD) para ajustar a reta mencionada.

\subsubsection{Método do Periodograma}

No início do Capítulo 3, foi apresentado que a densidade espectral de um processo de memória longa se aproxima de $C_{f} \omega^{1-2 H}$ quando a frequência tende a zero. Desde que a densidade espectral pode ser estimada pelo periodograma, o gráfico em escala logarítmica do periodograma contra a frequência deve se aproximar de uma reta com coeficiente de inclinação $(1-2 H)$ para frequências próximas de zero. Este método conhecido como Método do Periodograma pode ser utilizado para se estimar o valor de $H$.

A Figura 3.3 apresenta o gráfico do periodograma contra a frequência da série exemplo introduzida no início do capítulo. 


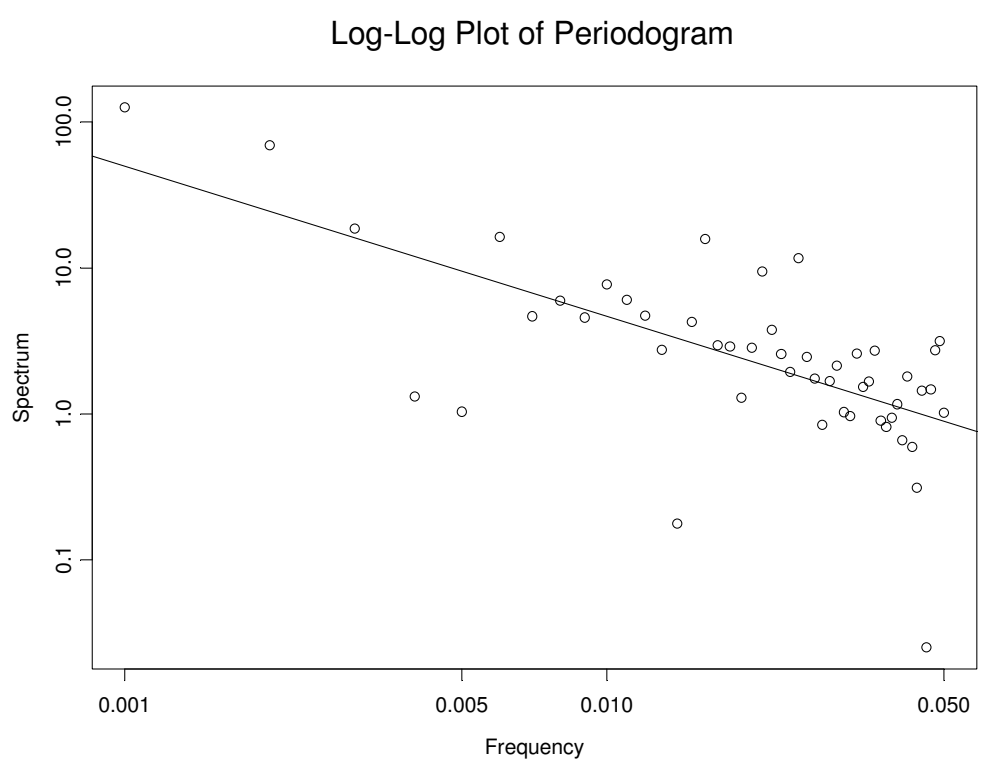

Figura 3.3 - Gráfico do Periodograma vs frequência

Novamente, o método LAD pode auxiliar na obtenção de estimativas mais robustas para o parâmetro de memória longa.

\subsubsection{Método de Whittle}

O método de Whittle para estimar $d$ é baseado na estimativa de máxima verossimilhança do processo fracionariamente integrado (3.4) no domínio da frequência. Pode ser demonstrado que os parâmetros do processo podem ser estimados através da minimização da versão discreta de

$$
Q(\theta)=\int_{-\pi}^{\pi} \frac{I(\omega)}{f(\theta, \omega)} d \omega
$$

em que $\theta$ é o vetor de parâmetros desconhecidos, incluindo o parâmetro de memória longa $d$, $I(\omega)$ é o periodograma de $z_{t}$ e $f(\theta, \omega)$ é a densidade espectral teórica de $z_{t}$.

\subsubsection{Estimador de Máxima Verossimilhança do Modelo ARFIMA}

O estimador de máxima verossimilhança (EMV) de um modelo ARFIMA (p,d,q) - equação (3.3), é obtido considerando-se a seguinte função de verossimilhança do processo $z=\left(z_{1}, \ldots z_{T}\right)$ 


$$
L\left(\eta, \sigma_{a}^{2}\right)=\left(2 \pi \sigma_{a}^{2}\right)^{-T / 2}\left(r_{0} \cdots r_{T-1}\right)^{-1 / 2} \exp \left[-\frac{1}{2 \sigma_{a}^{2}} \sum_{j=1}^{T}\left(z_{j}-\hat{z}_{j}\right)^{2} / r_{j-1}\right]
$$

em que $\eta=\left(d, \phi_{1}, \ldots, \phi_{p}, \theta_{1}, \ldots, \theta_{q}\right), \hat{z}_{j}$ para $j=1, \ldots, T$ são previsões um passo à frente $\mathrm{e}$ $r_{j-1}=\left(\sigma_{a}^{2}\right)^{-1} E\left(z_{j}-\hat{z}_{j}\right)^{2}$.

Os estimadores de máxima verossimilhança dos parâmetros são dados por

$$
\begin{gathered}
\hat{\sigma}_{M V}^{2}=T^{-1} S\left(\hat{\eta}_{M V}\right), \\
S\left(\hat{\eta}_{M V}\right)=\sum_{j=1}^{T}\left(z_{j}-\hat{z}_{j}\right)^{2} / r_{j-1},
\end{gathered}
$$

e $\hat{\eta}_{M V}$ é o valor de $\eta$ que minimiza

$$
l(\eta)=\ln (S(\eta) \mid T)+T^{-1} \sum_{j=1}^{T} \ln \left(r_{j-1}\right)
$$

Entretanto, o cálculo de $l(\eta)$ é bastante lento. Um procedimento alternativo é considerar uma aproximação dada por

$$
l(\eta) \simeq l_{*}(\eta)=\ln T^{-1} \sum_{j} \frac{I_{T}\left(\omega_{j}\right)}{2 \pi f\left(\omega_{j}, \eta\right)},
$$

em que

$$
I_{T}\left(\omega_{j}\right)=\frac{1}{T}\left|\sum_{t=1}^{T} z_{t} e^{-i t \omega_{j}}\right|^{2}
$$

é o periodograma dos dados,

$$
f\left(\omega_{j}, \eta\right)=\frac{\sigma_{a}^{2}}{2 \pi} \frac{\left|1-\theta_{1} e^{-i \omega_{j}}-\cdots-\theta_{q} e^{-q i \omega_{j}}\right|^{2}}{\left|1-\phi_{1} e^{-i \omega_{j}}-\cdots-\phi_{p} e^{-p i \omega_{j}}\right|^{2}}\left|1-e^{-i \omega_{j}}\right|^{-2 d}
$$


é a função densidade espectral do processo $z_{t}$ e $\sum_{j}$ é a soma sobre todas as frequências de Fourier, $\omega_{j}=\frac{2 \pi j}{T} \epsilon(-\pi, \pi]$.

Hannan (1973) e Fox e Taqqu (1986) mostram que:

- O estimador $\hat{\eta}_{M V}$ que minimiza a função (3.5) é consistente;

- Se $d>0, \hat{\eta}_{M V} \stackrel{D}{\rightarrow} N\left(\eta, T^{-1} A^{-1}(\eta)\right)$, em que $A(\eta)$ é uma matriz de ordem $(p+q+$ 1) $\times(p+q+1)$ com $(j, k)$-ésimo elemento dado por

$$
A_{j k}(\eta)=\frac{1}{4 \pi} \int_{-\pi}^{\pi} \frac{\partial \ln f(\lambda, \eta)}{\partial \eta_{j}} \frac{\partial \ln f(\lambda, \eta)}{\partial \eta_{k}} d \lambda ;
$$

- A variância $\sigma_{a}^{2}$ é estimada por

$$
\hat{\sigma}_{M V}^{2}=T^{-1} \sum_{j} \frac{I_{T}\left(\omega_{j}\right)}{2 \pi f\left(\omega_{j}, \hat{\eta}_{M V}\right)} .
$$

\subsection{Simulação}

Para estudar o desempenho dos testes e de cada um dos estimadores do parâmetro $\mathrm{d}$ de memória longa apresentados, foram efetuadas 1000 simulações com amostras de tamanho 1000 dos modelos ARFIMA( $(0, d, 0), \operatorname{ARFIMA}(1, \mathrm{~d}, 0)$ e $\operatorname{ARFIMA}(0, \mathrm{~d}, 1)$ para $\mathrm{d}=0,1,0,25$ e 0,45 e os parâmetros auto-regressivo e de médias móveis assumindo o valor de 0,5 .

Para cada um dos modelos simulados foram observadas as quantidades de testes que aceitaram e rejeitaram a hipótese nula de parâmetro de memória longa nulo dentro dos 1000 testes efetuados. Todos os modelos simulados apresentam $\mathrm{d}>0$ e é esperado, portanto, a rejeição da hipótese nula por todos os testes. Deste modo, foram observadas as quantidades de aceitação da hipótese nula dentre todos os testes tendo-se, assim, uma estimativa do erro de tipo II, ou seja, aceitar $\mathrm{H}_{0}$ sendo esta falsa.

Como o poder de um teste ( $\pi$ ) é definido por $\pi=1$ - erro de tipo II, o teste mais poderoso é aquele que apresenta a menor probabilidade de levar à conclusão de aceitar $\mathrm{H}_{0}$ sendo ela falsa, 
ou o teste que apresenta o menor erro de tipo II. A tabela abaixo apresenta os valores de erro do tipo II estimados dos testes R/S e GPH efetuados sobre as simulações.

Tabela 3.1 - Erros do tipo II dos testes R/S e GPH

Observa-se que para parâmetros de memória longa de pequena magnitude os testes apresentam elevados valores de erro de tipo II, possuindo, portanto, baixos poderes. Com a elevação do valor do parâmetro $d$, pode-se observar a diminuição do erro de tipo II. Modelos com a presença de fatores de auto-regressão apresentaram melhores resultados devido ao aumento da dependência na série.

A seguir, situa-se a comparação dos estimadores de diferentes métodos que será efetuada através das seguintes medidas:

- $\quad$ Média: $\bar{d}=\frac{\sum_{j=1}^{n} \hat{d}_{i}}{n}$;

- Erro Quadrático Médio: $\operatorname{EQ} \mathrm{M}(\hat{d})=\frac{\sum_{j=1}^{n}\left(\hat{d}_{i}-d\right)^{2}}{n}$.

Os métodos de GPH e de Whittle como apontado nas seções 3.2.2 e 3.3.3 serão utilizados para estimar apenas modelos $\operatorname{ARFIMA}(0, \mathrm{~d}, 0)$.

A tabela abaixo apresenta os resultados obtidos, sendo que os diferentes métodos de estimação estão separados por colunas enquanto que os diferentes modelos testados encontramse separados por linha. 
Tabela 3.2 - Resultado das Simulações dos Estimadores de Memória Longa

Para os modelos ARFIMA $(0, d, 0)$ pode-se observar que os estimadores do parâmetro de memória longa que mais se aproximam, em média, do valor real são: Whittle, GPH e EMV, sendo os métodos Whittle e EMV aqueles que apresentam os menores erros quadráticos médios.

Nota-se também que no caso dos modelos $\operatorname{ARFIMA}(1, \mathrm{~d}, 0)$ a definição do melhor estimador varia de acordo com a magnitude do parâmetro. Para o parâmetro de memória longa pequeno observa-se que o estimador que mais se aproximou em média e apresentou o menor erro quadrático médio foi aquele obtido através do método do periodograma. Para maiores parâmetros de memória longa o método R/S foi o que obteve a melhor estimativa, com baixos valores de erro quadrático médio.

O estimador de máxima verossimilhança, no caso dos modelos $\operatorname{ARFIMA}(0, \mathrm{~d}, 1)$, novamente apresentou melhores resultados, em comparação aos demais estimadores, tanto em média quanto em EQM.

Para facilitar a comparação entre os diversos estimadores, os gráficos abaixo apresentam o boxplot das estimativas obtidas pelas simulações separadas por parâmetro de memória longa utilizado para gerar as séries, modelo e método de estimação. 


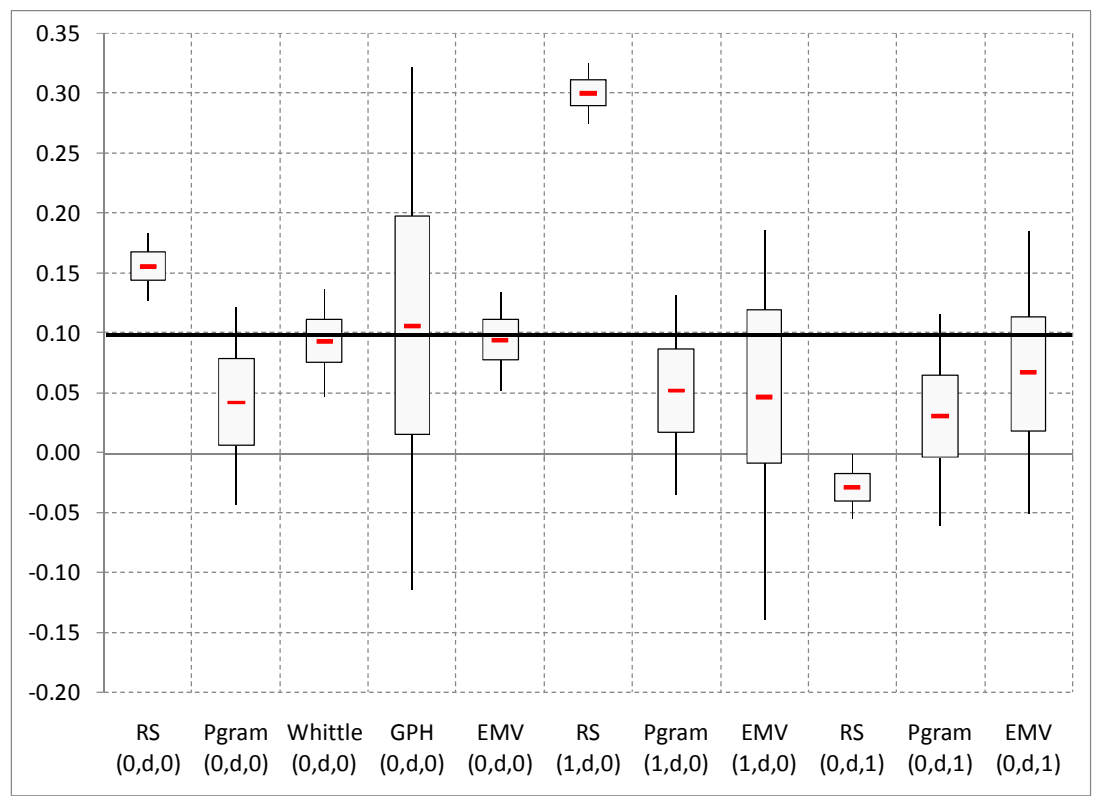

Figura 3.4 - boxplot dos valores $\hat{\mathrm{d}}$ das simulações das séries de memória longa com parâmetro d=0,1

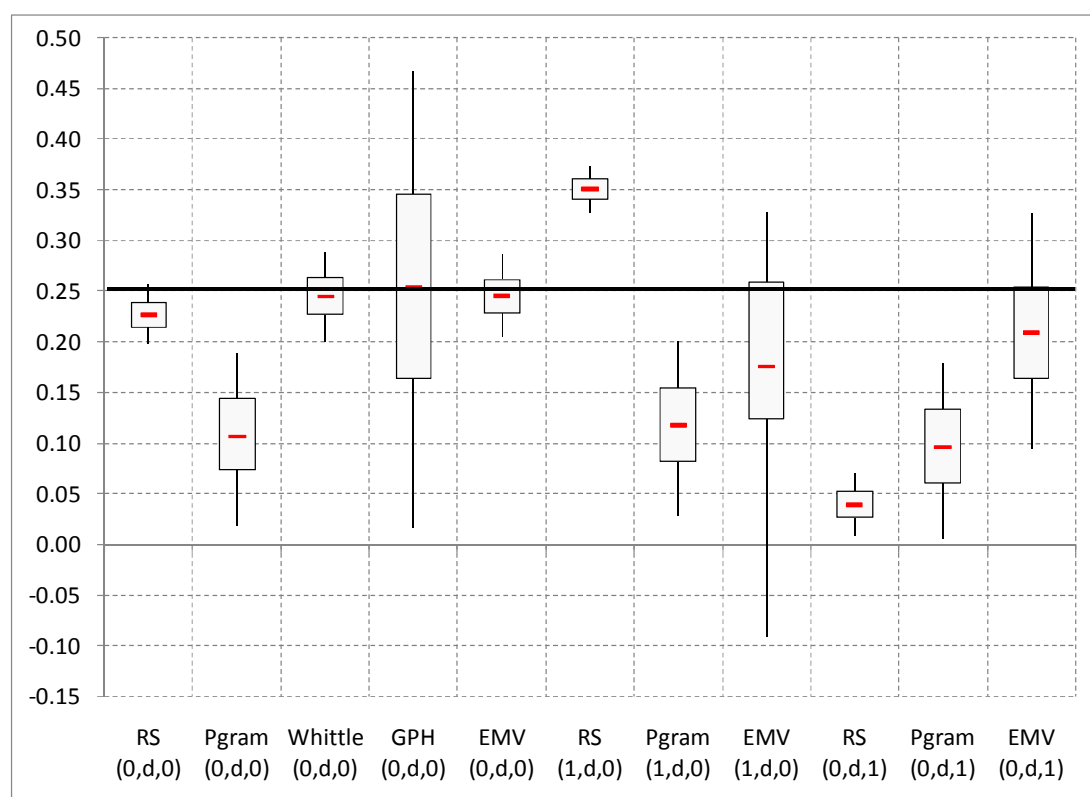

Figura 3.5 - boxplot dos valores d̂ das simulações das séries de memória longa com parâmetro d=0,25 


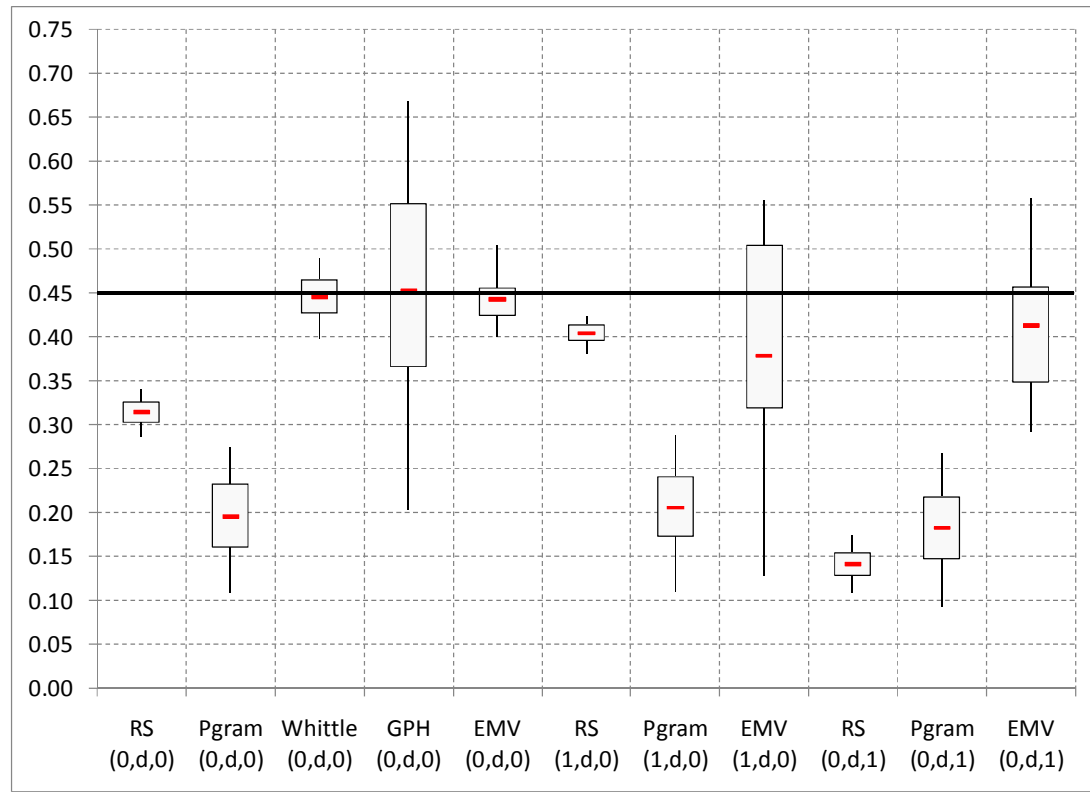

Figura 3.6 - boxplot dos valores d̂ das simulações das séries de memória longa com parâmetro d=0,45

Pelas dispersões observadas, nas figuras Figura 3.4, Figura 3.5 e Figura 3.6 confirma-se a boa performance dos estimadores de Whittle e de máxima verossimilhança para modelos $\operatorname{ARFIMA}(0, d, 0)$ que apresentam baixa variação em valores próximos ao valor real.

Na avaliação pela média e EQM para modelos $\operatorname{ARFIMA}(1, d, 0)$ com parâmetro mais elevado de memória longa o método R/S apresentou bons resultados, entretanto, observa-se pelos gráficos de dispersão que em nenhum momento este chegou a resultar no valor real (boxplot se localiza abaixo da reta do valor real 0,45$)$.

Através dos gráficos de boxplot, confirma-se, também, a obtenção de melhores resultados pelo estimador de máxima versossimilhança para modelos $\operatorname{ARFIMA}(0, d, 1)$. 


\section{Capítulo 4 Cointegração}

Os modelos de regressão linear e VAR apresentados na seção 2.4 são apropriados para modelagem de séries I(0) como retornos de preços. Se algumas ou todas as séries de um modelo de regressão forem I(1) os resultados estatísticos podem não ser válidos, como no caso de regressão espúria quando as séries não são estacionárias e não cointegradas.

Granger e Newbold (1974) verificaram através de simulações que dadas duas séries não correlacionadas, mas I(1), a regressão de uma sobre a outra tenderá a produzir uma relação aparentemente significativa. Considere os seguintes processos independentes e não cointegrados

$$
\begin{aligned}
& z_{1 t}=z_{1 t-1}+u_{1 t} \\
& z_{2 t}=z_{2 t-1}+u_{2 t}
\end{aligned}
$$

sendo $u_{t} \sim \mathrm{RBN}(0,1)$ a simulação de 250 observações pode ser observada no gráfico abaixo.

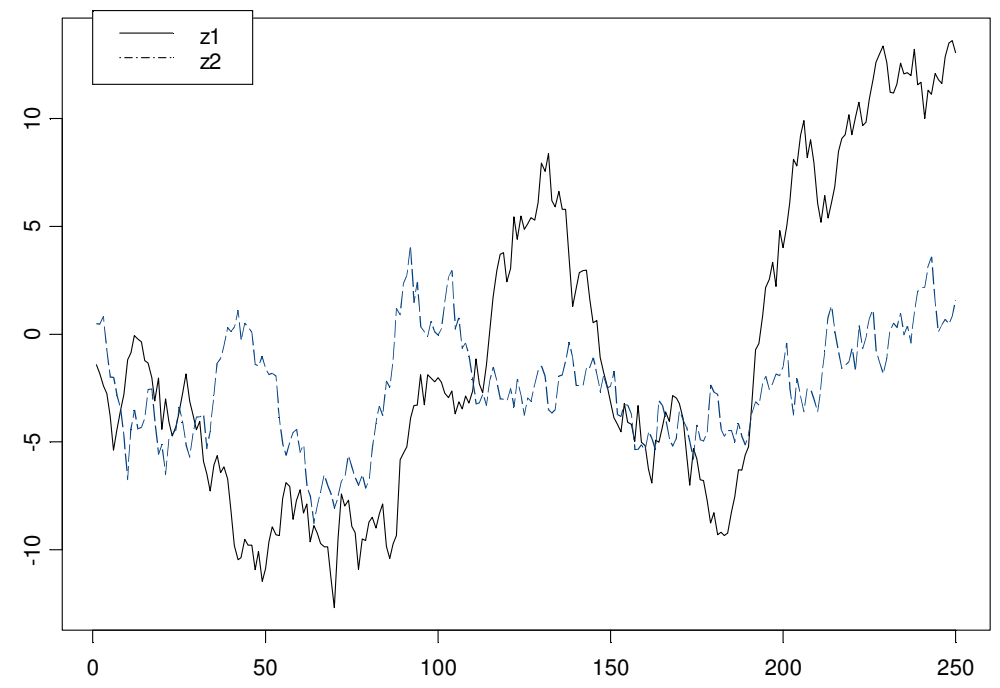

Figura 4.1 - Duas séries não estacionárias e não cointegradas

A regressão $z_{1 t}=\alpha+\beta z_{2 \mathrm{t}}+\varepsilon_{t}$ entre estas séries revela os seguintes resultados: 
Tabela 4.1 - Regressão entre duas series não estacionárias

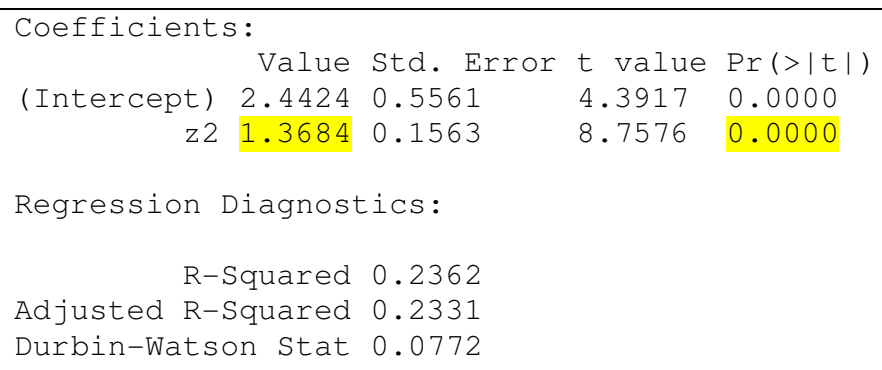

A estimativa 1,3684 ( $\mathrm{p}$ - valor $=0$ ) é significante e é observado um moderado valor de $\mathrm{R}^{2}$, no entanto, o baixo valor da statística de Durbin-Watson indica autocorrelação relevante residual.

A regressão entre as séries em primeiras diferenças revela a verdadeira relação entre as séries $(\mathrm{p}-$ valor $>0,05)$ :

Tabela 4.2 - Regressão entre duas series não estacionárias em primeiras diferenças

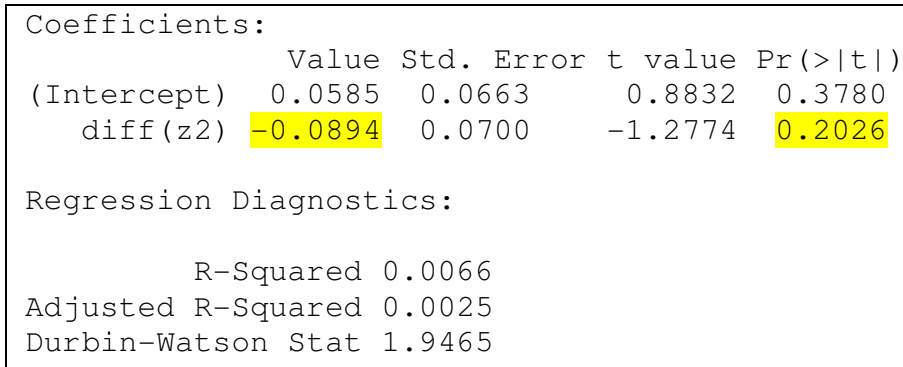

Usualmente, a Teoria Econômica estuda relações de equilíbrio entre séries, sendo diversas dessas séries não estacionárias, que necessitam, portanto, de um tratamento diferente daquele utilizado em séries estacionárias, levando ao desenvolvimento de modelos que consigam revelar estas relações entre as séries não estacionárias.

\subsection{Modelos de Cointegração}

Sendo $Z_{t}=\left(z_{1 t}, z_{2 t}, \ldots, z_{n t}\right)^{\prime}$, um vetor $(n \times 1)$ de séries temporais $I(d) d \in \mathbb{N}, Z_{t}$ é considerado cointegrado de ordem $(d, b)$, ou seja, $Z_{t} \sim$ C. I. $(d, b)$, se existe um vetor $(n \times 1)$ $\beta=\left(\beta_{1}, \beta_{2}, \ldots, \beta_{n}\right)^{\prime}$, chamado de vetor de cointegração, tal que

$$
\beta^{\prime} Z_{t}=\beta_{1} z_{1 t}+\beta_{2} z_{2 t}+\cdots+\beta_{\mathrm{n}} z_{n t} \sim \mathrm{I}(\mathrm{d}-\mathrm{b}), \quad d \geq b>0
$$


Engle e Granger (1987) fornecem um exemplo considerando $n=2$ e as séries $z_{1 t}$ e $z_{2 t}$ dadas por

$$
\begin{gathered}
z_{1 t}+\gamma z_{2 t}=u_{t} \\
u_{t}=\phi_{1} u_{t-1}+\varepsilon_{1 t} \\
z_{1 t}+\alpha z_{2 t}=v_{t} \\
v_{t}=\phi_{2} v_{t-1}+\varepsilon_{2 t}
\end{gathered}
$$

sendo $\varepsilon_{i t}$ independentes e normais com média zero e $E\left(\varepsilon_{i t} \varepsilon_{j t}\right)=0, \mathrm{i}, \mathrm{j}=1,2$ e $\phi_{\mathrm{i}}$ 's não nulos. Tem-se os seguintes casos:

- $\quad \phi_{\mathrm{i}}<1, i=1,2 . z_{1 t}$ e $z_{2 t}$ são I(0) e $\alpha$ e $\gamma$ não são identificáveis;

- $\phi_{1}=1$ e $\phi_{2}<1 . z_{1 t}$ e $z_{2 t}$ são I(1) e $(1, \alpha)^{\prime}$ é o vetor de cointegração, identificável;

- $\phi_{1}<1$ e $\phi_{2}=1$. $z_{1 t}$ e $z_{2 t}$ são I(1) e $(1, \gamma)^{\prime}$ é o vetor de cointegração, identificável.

$\mathrm{O}$ vetor de cointegração $\beta$ não é único, pois, para qualquer escalar $c \neq 0, c \beta$ também é um vetor de cointegração, ou seja, $c \beta^{\prime} Z_{t} \sim \mathrm{I}(0)$. Por este motivo, algumas suposições de normalização são necessárias, sendo usualmente um dos parâmetros para normalizar o vetor de cointegração, tornando-se $\beta=\left(1,-\beta_{2}, \ldots,-\beta_{\mathrm{n}}\right)^{\prime}$ de modo que

$$
\beta^{\prime} z_{t}=z_{1 t}-\beta_{2} z_{2 t}-\cdots-\beta_{\mathrm{n}} z_{n t} \sim \mathrm{I}(0)
$$

ou

$$
z_{1 t}=\beta_{2} z_{2 t}+\cdots+\beta_{\mathrm{n}} z_{n t}+u_{t}
$$

com $u_{t} \sim I(0)$, conhecido como resíduo de cointegração. Como, no longo prazo, tem-se $u_{t}=0$, a relação de longo prazo entre as variáveis é

$$
z_{1 t}=\beta_{2} z_{2 t}+\cdots+\beta_{\mathrm{n}} z_{n t}
$$

Quando $\mathrm{n}>2$, podem existir $\mathrm{r}$ vetores de cointegração $(0<\mathrm{r}<n)$, que, organizados numa matriz B de ordem $(n \times r)$, com posto $r$, chamado de posto de cointegração, possui a seguinte forma 


$$
\mathrm{B}^{\prime} Z_{t}=\left[\begin{array}{cccc}
\beta_{11} & \beta_{12} & \ldots & \beta_{1 \mathrm{n}} \\
\beta_{21} & \beta_{22} & \ldots & \beta_{2 \mathrm{n}} \\
\vdots & & \\
\beta_{\mathrm{r} 1} & \beta_{\mathrm{r} 2} & \ldots & \beta_{\mathrm{rn}}
\end{array}\right]\left[\begin{array}{c}
z_{1 t} \\
z_{2 t} \\
\vdots \\
z_{n t}
\end{array}\right]=\left[\begin{array}{c}
\mathrm{u}_{1 \mathrm{t}} \\
\mathrm{u}_{2 \mathrm{t}} \\
\vdots \\
\mathrm{u}_{\mathrm{rt}}
\end{array}\right] .
$$

Um sistema para $Z_{t}=\left(z_{1 t}, z_{2 t}\right)^{\prime}$ com vetor de cointegração $\beta=\left(1,-\beta_{2}\right)^{\prime}$ pode ser simulado através da seguinte representação triangular:

$$
\begin{aligned}
& z_{1 t}=\beta_{2} z_{2 t}+u_{t} \\
& z_{2 t}=z_{2 t-1}+v_{t}
\end{aligned}
$$

com $u_{t}$ e $v_{t} \sim \mathrm{I}(0)$. O gráfico abaixo apresenta a evolução de duas séries geradas pelo sistema acima, considerando $\beta_{2}=0,8$.

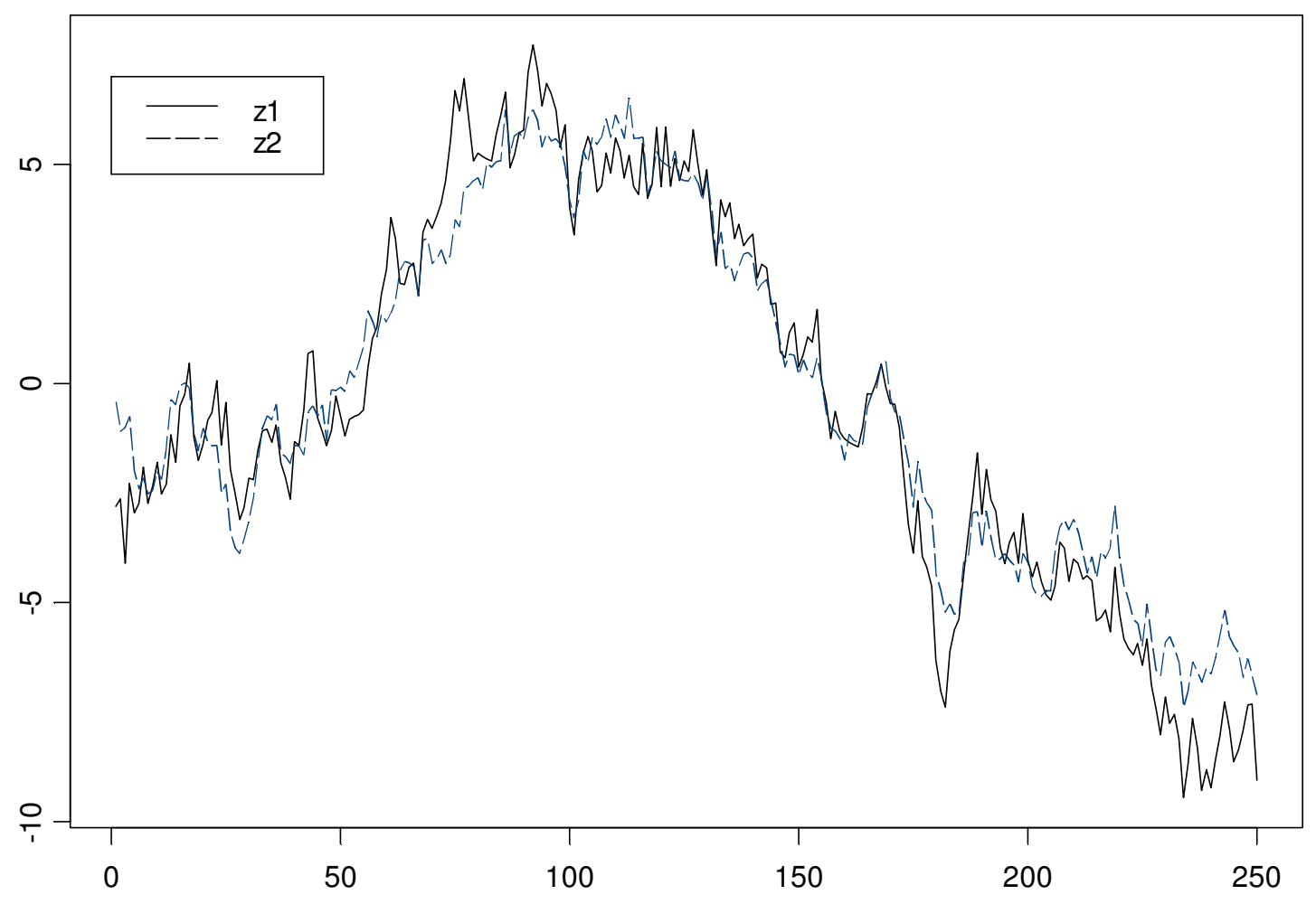

Figura 4.2 - Simulação de séries cointegradas 


\subsection{Tendências Comuns}

Alguns exemplos de cointegração podem ser observados em modelos nas áreas de economia e finanças, geralmente associados a teorias que buscam as relações de equilíbrio entre séries. Em economia modelos de renda, consumo, inflação, taxas de juros e investimentos são exemplos de séries que possuem uma tendência comum, assim como taxa de câmbio nominal, estrangeira e inflação em modelos de poder de compra. Estas relações de equilíbrio são chamadas de relações de equilíbrio de longo prazo devido a lenta resposta das forças econômicas aos desequilíbrios.

Em finanças, cointegração pode ser avaliada em relações de alta frequência e de baixa frequência. Relações de alta frequência são relacionadas a teorias de arbitragem que explicam que ativos iguais tendem a ser vendidos pelo mesmo preço, como, por exemplo, a venda de um mesmo produto em diferentes mercados, ou a relação entre preços futuros e a vista de um mesmo ativo. Relações de baixa frequência são motivadas por teorias econômicas de equilíbrio ligando preços de ações a análises de empresas, como, por exemplo, a teoria de que o preço de uma ação representa o valor presente das projeções de pagamentos de lucros ou dividendos.

Se uma série temporal $Z_{t}$ vetorial $(\mathrm{n} \times 1)$, possuir $\mathrm{r}$ vetores de cointegração $(0<\mathrm{r}<n)$, exitirão, portanto, $n-r$ tendências estocásticas comuns.

Para exemplificar, considere $\mathrm{Z}_{\mathrm{t}}=\left(\mathrm{z}_{1 \mathrm{t}}, \mathrm{z}_{2 \mathrm{t}}\right)^{\prime} \sim I(1), \mathrm{u}_{\mathrm{t}}=\left(\mathrm{u}_{1 \mathrm{t}}, \mathrm{u}_{2 \mathrm{t}}, \mathrm{u}_{3 \mathrm{t}}\right)^{\prime} \sim \mathrm{I}(0)$ e que $\mathrm{Z}_{\mathrm{t}}$ é cointegrado com vetor de cointegração $\beta=\left(1,-\beta_{2}\right)^{\prime}$. Esta relação pode ser expressa na forma

$$
\begin{gathered}
z_{1 t}=u_{2 t}+\beta_{2} \sum_{\mathrm{k}=1}^{\mathrm{t}} u_{1 k}, \\
z_{2 t}=u_{3 t}+\sum_{\mathrm{k}=1}^{\mathrm{t}} u_{1 k} .
\end{gathered}
$$

A tendência comum do exemplo é $\sum_{\mathrm{k}=1}^{\mathrm{t}} u_{1 k}$, que é eliminada pela relação de cointegração

$$
\beta^{\prime} Z_{t}=z_{1 t}-\beta_{2} z_{2 t}=u_{2 t}+\beta_{2} \sum_{\mathrm{k}=1}^{\mathrm{t}} u_{1 k}-\beta_{2}\left(u_{3 t}+\sum_{\mathrm{k}=1}^{\mathrm{t}} u_{1 k}\right)=u_{2 t}-\beta_{2} u_{3 t} \sim \mathrm{I}(0)
$$


Outro fato relevante, apontado por Alexander (2001), é o da cointegração entre séries não implicar em elevada correlação entre as mesmas. Supondo

$$
\begin{aligned}
& z_{1 t}=u_{t}+\varepsilon_{1 t} \\
& z_{2 t}=u_{t}+\varepsilon_{2 t} \\
& u_{t}=u_{t-1}+\varepsilon_{t}
\end{aligned}
$$

com $\varepsilon_{t}$ 's i.i.d., nota-se que $z_{1 t}$ e $z_{2 t}$ são I(1) pelo fato de $u_{t}$ ser um passeio aleatório, além de serem cointegradas pois $z_{1 t}-z_{2 t}=\varepsilon_{1 t}-\varepsilon_{2 t} \sim I(0)$ e possuirem a tendência estocástica comum $u_{t}$.

Declarando-se $\operatorname{var}\left(\varepsilon_{t}\right)=\sigma^{2}, \operatorname{var}\left(\varepsilon_{1 t}\right)=\sigma_{1}^{2}$ e $\operatorname{var}\left(\varepsilon_{2 t}\right)=\sigma_{2}^{2}$ e notando-se que $\Delta z_{1 t}=\varepsilon_{t}+$ $\varepsilon_{1, t}-\varepsilon_{1, t-1}$ segue-se que $\operatorname{var}\left(\Delta z_{1 t}\right)=\sigma^{2}+2 \sigma_{1}^{2}$. Analogamente tem-se $\operatorname{var}\left(\Delta z_{2 t}\right)=\sigma^{2}+2 \sigma_{2}^{2}$.

O coeficiente de correlação

$$
\rho\left(\Delta z_{1 t}, \Delta z_{2 t}\right)=\frac{\sigma^{2}}{\sqrt{\left(\sigma^{2}+2 \sigma_{1}^{2}\right)\left(\sigma^{2}+2 \sigma_{2}^{2}\right)}}
$$

será baixo se $\sigma^{2} \ll \sigma_{1}^{2}$ ou $\sigma^{2} \ll \sigma_{2}^{2}$.

\subsection{Modelo de Correção de Erros}

Considere $\mathrm{Z}_{\mathrm{t}}=\left(\mathrm{z}_{1 \mathrm{t}}, \mathrm{z}_{2 \mathrm{t}}\right)^{\prime}$, um vetor de séries temporais $\mathrm{I}(1)$, cointegrado pelo vetor de cointegração $\beta=\left(1,-\beta_{2}\right)^{\prime}$. Engle e Granger (1987) mostram que esta relação de cointegração implica a existência de um modelo de correção de erros (ECM) da forma

$$
\begin{aligned}
& \Delta z_{1 t}=\alpha_{1}\left(z_{1 t-1}-\beta_{2} z_{2 t-1}\right)+a_{11} \Delta z_{1 t-1}+a_{12} \Delta z_{2 t-1}+\varepsilon_{1 t} \\
& \Delta z_{2 t}=\alpha_{2}\left(z_{1 t-1}-\beta_{2} z_{2 t-1}\right)+a_{21} \Delta z_{1 t-1}+a_{22} \Delta z_{2 t-1}+\varepsilon_{2 t} .
\end{aligned}
$$

Este é um modelo VAR(1) ajustado às primeiras diferenças com um termo de correção de erro adicionado. Os parâmetros $\alpha_{1}$ e $\alpha_{2}$ são relacionados à velocidade de ajuste da relação de equilíbrio, caso ambos sejam nulos, não existirá relação de longo prazo. O modelo de correção 
de erros relaciona a relação de equilíbrio de longo prazo e os ajustes de curto prazo que descrevem como as variáveis se movimentam enquanto não seguem o equilíbrio.

Pode-se escrever o modelo da seguinte forma:

$$
\Delta Z_{t}=\alpha \beta^{\prime} Z_{t-1}+\phi_{1} \Delta Z_{t-1}+\varepsilon_{t}
$$

em que, $\alpha=\left(\begin{array}{l}\alpha_{1} \\ \alpha_{2}\end{array}\right), \phi_{1}=\left(\begin{array}{ll}a_{11} & a_{12} \\ a_{21} & a_{22}\end{array}\right)$ e $\varepsilon_{t}=\left(\begin{array}{l}\varepsilon_{1 t} \\ \varepsilon_{2 t}\end{array}\right)$.

Generalizando para um modelo VAR(p) tem-se

$$
\Delta Z_{t}=\phi_{0}+\alpha \beta^{\prime} Z_{t-1}+\phi_{1} \Delta Z_{t-1}++\phi_{2} \Delta Z_{t-2}+\cdots++\phi_{\mathrm{p}} \Delta Z_{t-p}+\varepsilon_{t}
$$

Para exemplificar, considere $\beta=(1,-1)^{\prime}$ e a seguinte relação de equilíbrio

$$
z_{2 t}=z_{1 t}+\mu+u_{t}
$$

em que $\mu$ é a media de $z_{2 t}-z_{1 t}$ e $u_{t}$ é o erro de desequilíbrio (resíduo de cointegração). Suponha o modelo de correção de erros

$$
\begin{array}{ll}
\Delta z_{1 t}=c_{1}+\alpha_{1}\left(z_{2 t-1}-z_{1 t-1}-\mu\right)+\varepsilon_{1 t}, & c_{1}>0 \\
\Delta z_{2 t}=c_{2}+\alpha_{2}\left(z_{2 t-1}-z_{1 t-1}-\mu\right)+\varepsilon_{2 t}, & c_{2}>0 .
\end{array}
$$

A primeira equação relaciona a variação de $z_{1 t}$ com o desvio defasado de $z_{2 t-1}-z_{1 t-1}-\mu$ e a segunda equação apresenta a variação de $z_{2 t}$ em relação ao mesmo desvio. Considerando as duas equações e $\alpha_{1}=0,5$ e $\alpha_{2}=0$ tem-se

$$
\begin{gathered}
\Delta z_{1 t}=c_{1}+0,5\left(z_{2 t-1}-z_{1 t-1}-\mu\right)+\varepsilon_{1 t} \\
\Delta z_{2 t}=c_{2}+\varepsilon_{2 t} .
\end{gathered}
$$

Note que $E\left[\Delta z_{1 t} \mid Z_{t-1}\right]=c_{1}+0,5\left(z_{2 t-1}-z_{1 t-1}-\mu\right)$ e $E\left[\Delta z_{2 t} \mid Z_{t-1}\right]=c_{2}$. Existem três situações:

- $z_{2 t-1}-z_{1 t-1}-\mu=0$, portanto $E\left[\Delta z_{1 t} \mid Z_{t-1}\right]=c_{1}$ e $E\left[\Delta z_{2 t} \mid Z_{t-1}\right]=c_{2}$, sendo $c_{1} \mathrm{e}$ $c_{2}$ as taxas de crescimento das variáveis no equilíbrio de longo prazo; 
- $z_{2 t-1}-z_{1 t-1}-\mu>0$, portanto $E\left[\Delta z_{1 t} \mid Z_{t-1}\right]=c_{1}+0,5\left(z_{2 t-1}-z_{1 t-1}-\mu\right)>c_{1}$ indicando que $z_{2 t}$ cresceu mais rápido do que sua média de longo prazo e o modelo prevê um crescimento mais rápido para $z_{1 t}$ (sendo 0,5 o coeficiente da velocidade de resposta ao desiquilíbrio) de forma a restaurar o equilíbrio;

- $z_{2 t-1}-z_{1 t-1}-\mu<0$, portanto $E\left[\Delta z_{1 t} \mid Z_{t-1}\right]=c_{1}+0,5\left(z_{2 t-1}-z_{1 t-1}-\mu\right)<c_{1}$ indicando que $z_{2 t}$ cresceu muito abaixo do que sua média de longo prazo e o modelo prevê um crescimento inferior para $z_{1 t}$ de forma a restaurar o equilíbrio.

Estes casos ilustram a razão pela qual o modelo é conhecido como correção de erros. Quando as variáveis se encontram fora da relação de equilíbrio de longo prazo há uma força que faz com que elas retornem ao equilíbrio e a velocidade com que isto ocorre é determinada pela magnitude do parâmetro $\alpha$. No exemplo acima $\alpha=0,5$ indicando que metade do desequilíbrio é corrigido a cada período, caso $\alpha=1$ a correção a cada período seria de mesma magnitude que o erro de desequilíbrio e caso $\alpha>1$ a correção ultrapassa o valor do erro a cada período.

\subsection{Testes para Cointegração}

Sendo $\mathrm{Z}_{\mathrm{t}}=\left(\mathrm{z}_{1 \mathrm{t}}, \mathrm{z}_{2 \mathrm{t}}, \ldots, \mathrm{z}_{\mathrm{nt}}\right)^{\prime}$ um vetor de séries temporais $\mathrm{I}(1)$. Como visto, este será cointegrado com $r$ vetores de cointegração se existir uma matriz $B^{\prime},(r \times \mathrm{n})$, tal que

$$
\mathrm{B}^{\prime} Z_{t}=\left[\begin{array}{c}
\beta^{(1)^{\prime}} Z_{t} \\
\beta^{(2)^{\prime}} Z_{t} \\
\vdots \\
\beta^{(\mathrm{r})^{\prime}} Z_{t}
\end{array}\right]=\left[\begin{array}{c}
\mathrm{u}_{\mathrm{t}}^{(1)} \\
\mathrm{u}_{\mathrm{t}}^{(2)} \\
\vdots \\
\mathrm{u}_{\mathrm{t}}^{(\mathrm{r})}
\end{array}\right] \sim \mathrm{I}(0) .
$$

Testes para cointegração podem ser efetuados como teste da existência de equilíbrios de longo prazo entre as variáveis, o que leva a duas situações:

- Há pelo menos um vetor de cointegração;

- Há possíveis $\mathrm{r}$ vetores de cointegração $0 \leq \mathrm{r}<n$.

O primeiro caso foi originalmente tratado por Engle e Granger (1986) e um teste de duas iterações baseado nos resíduos do modelo foi desenvolvido. O segundo foi primeiramente 
considerado por Johansen (1988) que desenvolveu um processo sofisticado para determinar o número de relações de cointegração através de técnicas de máxima verossimilhança.

\subsubsection{Procedimento de Engle e Granger}

O teste pode ser efetuado em duas situações: quando o vetor de cointegração é pré-definido e quando o vetor de cointegração é estimado.

\section{Vetor de cointegracão pré-definido}

Sendo $Z_{t}$ um vetor $(n \times 1)$ de séries temporais $I(1), \beta$ um vetor $(n \times 1)$ de cointegração prédefinido e $u_{t}=\beta^{\prime} Z_{t}$ o resíduo de cointegração pré-definido. As hipóteses a serem testadas são

$$
\begin{gathered}
\mathrm{H}_{0}: u_{t}=\beta^{\prime} Z_{t} \sim \mathrm{I}(1) \text { (Não há cointegração) } \\
\mathrm{H}_{1}: u_{t}=\beta^{\prime} Z_{t} \sim \mathrm{I}(0) \text { (há cointegração). }
\end{gathered}
$$

Qualquer teste de raiz unitária pode ser utilizado para avaliar as hipóteses acima. O teste mais utilizado é o ADF. Caso o teste rejeite a presença de raiz unitária nos resíduos, a relação de cointegração é aceita.

\section{$\underline{\text { Vetor de cointegração estimado }}$}

Seja $Z_{t}$ um vetor $(n \times 1)$ de séries temporais $I(1), \beta$ um vetor $(n \times 1)$ de cointegração desconhecido e considere as hipóteses a serem testadas equivalentes às do caso anterior. Como $\beta$ é desconhecido, para que o teste possa ser efetuado, é necessária a estimação do vetor de cointegração.

Uma normalização comum para estimar o vetor $\beta$ é definir o primeiro elemento de $Z_{t}$ como variável dependente, ou seja, $\mathrm{Z}_{\mathrm{t}}=\left(\mathrm{z}_{1 \mathrm{t}}, \mathrm{Z}_{2 \mathrm{t}}^{\prime}\right)^{\prime}$ em que $\mathrm{Z}_{2 \mathrm{t}}=\left(\mathrm{z}_{2 \mathrm{t}}, \ldots, \mathrm{z}_{\mathrm{nt}}\right)^{\prime}$ é um vetor $((\mathrm{n}-1) \times$ 1) e o vetor de cointegração é escrito na forma $\beta=\left(1,-\beta_{2}^{\prime}\right)^{\prime}$. Engle e Granger propoem a estimação de $\beta_{2}$ por mínimos quadrados da regressão

$$
\mathrm{z}_{1 \mathrm{t}}=\mathrm{c}+\beta^{\prime}{ }_{2} \mathrm{z}_{2 \mathrm{t}}+\mathrm{u}_{\mathrm{t}}
$$

obtendo-se as estimativas $\widehat{c}$ e $\widehat{\beta}_{2}$ e realizando-se o teste de raiz unitária sobre o resíduo 


$$
\hat{\mathrm{u}}_{\mathrm{t}}=\mathrm{z}_{1 \mathrm{t}}-\hat{\mathrm{c}}-\hat{\beta}_{2}^{\prime} \mathrm{Z}_{2 \mathrm{t}}
$$

Contudo, Phillips e Ouliaris (1990) mostram que o teste ADF sobre os resíduos não possuem a mesma distribuição sob a hipótese nula de não cointegração. Devido ao fenômeno de regressão espúria sob a hipóstese nula, a distribuição assintótica do ADF torna-se uma função do processo de Wiener dos termos determinísticos utilizados para estimar $\beta$ e do número de variáveis $n-1$. Essas distribuições são conhecidas como Phillips-Ouliaris (PO).

\subsubsection{Regressão e modelo de correção de erros}

Estimadores de mínimos quadrados podem ser utilizados para estimar consistentemente o vetor de cointegração. Stock (1987) e Phillips (1991) apontam os seguintes comportamentos para $\widehat{\beta}_{2}$ quando as séries são cointegradas:

- $T\left(\widehat{\beta}_{2}-\beta_{2}\right)$ converge para uma variável aleatória com distribuição diferente da normal não possuindo, necessariamente, média zero;

- Os estimadores de mínimos quadrados são consistentes e convergem para o valor real a uma taxa de $T$ em vez de $T^{1 / 2}$;

- $\widehat{\beta}_{2}$ é consistente mesmo que $Z_{2 t}$ seja correlacionado com $u_{t}$;

- A distribuição assintótica de $T\left(\widehat{\beta}_{2}-\beta_{2}\right)$ é, em geral, assintoticamente viesada e não normal, portanto, os erros padrão da regressão não são válidos;

- Mesmo que o viés assintótico tenda a zero a medida que $T$ aumente, os estimadores em pequenas amostras podem ser substancialmente viesadas.

Tais considerações mostram que os estimadores de mínimos quadrados podem ser aprimorados, como sugerido por Stock e Watson (1993). Eles sugerem um método para se obter um estimador assintoticamente eficiente para o vetor de cointegração normalizado e sua variância assintótica através do seguinte processo: 
- Aumentar a regressão de $\mathrm{z}_{1, \mathrm{t}}$ por $\mathrm{Z}_{2, \mathrm{t}}$ com termos determinísticos $\mathrm{D}_{\mathrm{t}}$ e $p$ diferenças $\Delta \mathrm{Z}_{2, \mathrm{t}}$ anteriores e posteriores

$$
\mathrm{z}_{1, \mathrm{t}}=\gamma^{\prime} \mathrm{D}_{\mathrm{t}}+\beta^{\prime}{ }_{2} \mathrm{Z}_{2, \mathrm{t}}+\sum_{k=-p}^{p} \psi_{\mathrm{k}}^{\prime} \Delta \mathrm{z}_{2, \mathrm{t}-\mathrm{k}}+\mathrm{u}_{\mathrm{t}}
$$

- Estimar a regressão por mínimos quadrados. O estimador resultante é chamado estimador de mínimos quadrados ordinário dinâmico (Dynamic Ordinary Least

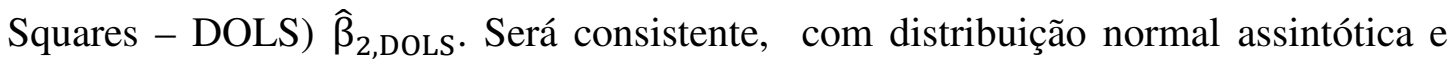
eficiente sob certas condições;

- Erros padrões dos estimadores válidos assintoticamente, obtidos através do erros padrões da regressão multiplicados por

$$
\left(\frac{\widehat{\sigma}_{\mathrm{u}}^{2}}{\widehat{\operatorname{lrv}}\left(\mathrm{u}_{\mathrm{t}}\right)}\right)^{1 / 2}
$$

em que $\widehat{\sigma}_{u}^{2}$ é o estimador de mínimos quadrados da variância de $\widehat{u}_{t}$ e $\widehat{\operatorname{lrv}}\left(\mathrm{u}_{\mathrm{t}}\right)$ é o estimador da variância de longo prazo de $\hat{\mathrm{u}}_{\mathrm{t}}$.

Para estimar o modelo de correção de erros (4.1) equivalente, a estimativa eficiente para o vetor de cointegração $\widehat{\beta}=\left(1,-\widehat{\beta}^{\prime}{ }_{2}\right)^{\prime}$ pode ser considerada como verdadeira de forma que o erro estimado de desequilíbrio $\mathrm{z}_{1 \mathrm{t}}-\widehat{\beta}^{\prime}{ }_{2} \mathrm{Z}_{2 \mathrm{t}}$ também poderá ser considerado como conhecido. Como todas as variáveis no modelo de correção de erros são $I(0)$, as regressões podem ser consitentemente estimadas pelo método dos mínimos quadrados.

\subsubsection{Procedimento de Johansen}

Considere o modelo $\operatorname{VAR}(\mathrm{p})$ para um $\mathrm{Z}_{\mathrm{t}}=\left(\mathrm{z}_{1 \mathrm{t}}, \mathrm{z}_{2 \mathrm{t}}, \ldots, \mathrm{z}_{\mathrm{nt}}\right)^{\prime}$ um vetor $(\mathrm{n} \times 1)$

$$
Z_{t}=\Phi D_{t}+\Pi_{1} Z_{t-1}+\Pi_{2} Z_{t-2}+\cdots+\Pi_{\mathrm{p}} Z_{t-p}+\varepsilon_{\mathrm{t}}, \quad t=1, \ldots, T
$$

este modelo será estável se todas as raízes da equação abaixo estiverem fora do círculo unitário

$$
\operatorname{det}\left(I_{n}-\Pi_{1} z-\Pi_{2} z^{2}-\cdots-\Pi_{p} z^{p}\right)=0
$$


Caso uma das raízes da equação estiver sobre o círculo unitário, algumas, talvez todas, variáveis aleatórias de $Z_{t}$ são I(1) e, possivelmente, cointegradas. No caso das variáveis serem realmente I(1) e, possivelmente, cointegradas o modelo VAR(p) não é a melhor representação pois as relações de cointegração não estão indicadas, como no modelo de correção de erros

$$
\Delta Z_{t}=\Phi D_{t}+\Pi Z_{t-1}+\Gamma_{1} \Delta Z_{t-1}+\cdots+\Gamma_{\mathrm{p}-1} \Delta Z_{t-p+1}+\varepsilon_{\mathrm{t}}
$$

em que $\Pi=\Pi_{1}+\cdots+\Pi_{\mathrm{p}}-\mathrm{I}_{\mathrm{n}}$ e $\Gamma_{\mathrm{k}}=-\sum_{\mathrm{i}=\mathrm{k}+1}^{\mathrm{p}} \Pi_{\mathrm{i}}, k=1, \ldots, p-1$. A matriz $\Pi$ é chamada matriz de impacto no longo prazo e $\Gamma_{\mathrm{k}}$ são as matrizes de impacto no curto prazo.

Neste modelos $\Delta Z_{t}$ e suas defasagens são $\mathrm{I}(0)$ e o termo $\Pi Z_{t-1}$ é o único que pode conter séries I(1) e apenas no caso deste termo ser I(0) tem-se $\Delta Z_{t} \mathrm{I}(0)$, ou seja, $\Pi Z_{t-1}$ contém as relações de cointegração, caso existam.

Se o modelo $\operatorname{VAR}(\mathrm{p})$ indica a presença de raízes unitárias a matriz $\Pi$ será singular e, portanto, possui um posto $r<n$. Tem-se dois casos:

- $\quad r=0$, implicando que $\Pi=0$ e $Z_{t}$ é I(1) e não cointegrada;

- $0<\mathrm{r}<n$, implicando que $Z_{t}$ é I(1) com $\mathrm{r}$ vetores de cointegração linearmente independentes e $\mathrm{n}-\mathrm{r}$ tendências estocásticas comuns. A matriz $\Pi$ pode ser escrita como o produto $\Pi_{\mathrm{n} \times \mathrm{n}}=\alpha_{\mathrm{n} \times \mathrm{r}} \beta^{\prime}{ }_{\mathrm{r} \times \mathrm{n}}$ em que $\alpha$ e $\beta$ são matrizes $\mathrm{n} \times \mathrm{r}$ com postos $\mathrm{r}$. As linhas de $\beta^{\prime}$ são os $r$ vetores de cointegração e os elementos de $\alpha$ distribuem o impacto dos vetores de cointegração na evolução de $\Delta Z_{t}$ e o modelo de correção de erros possuirá a seguinte forma

$$
\Delta Z_{t}=\Phi D_{t}+\alpha \beta^{\prime} Z_{t-1}+\Gamma_{1} \Delta Z_{t-1}+\cdots+\Gamma_{\mathrm{p}-1} \Delta Z_{t-p+1}+\varepsilon_{\mathrm{t}}
$$

sendo $\beta^{\prime} Z_{t-1} \mathrm{I}(0)$.

O seguinte procedimento de modelagem de cointegração é conhecido como método de Johansen

- Verificar a ordem de integração das séries;

- Ajustar um modelo VAR(p) para $Z_{t}$; 
- Construir testes de razão de verossimilhança para o posto de П, ou seja, o número de vetores de cointegração;

- Dados os vetores de cointegração normalizados estimar o modelo de correção de erros, através do método de máxima verossimilhança.

De acordo com Johansen(1995), os termos determinísticos estão restritos à forma

$$
\Phi D_{t}=\mu_{\mathrm{t}}=\mu_{0}+\mu_{1} \mathrm{t}
$$

e podem ser classificados em 5 casos:

- Caso $\mu_{\mathrm{t}}=0$, o modelo de correção de erros será

$$
\Delta Z_{t}=\alpha \beta^{\prime} Z_{t-1}+\Gamma_{1} \Delta Z_{t-1}+\cdots+\Gamma_{\mathrm{p}-1} \Delta Z_{t-p+1}+\varepsilon_{\mathrm{t}}
$$

e todas as series em $Z_{t}$ são I(1) sem “drift" e as relações de cointegração $\beta^{\prime} Z_{t}$ possuem média zero.

- Caso $\mu_{\mathrm{t}}=\mu_{0}=\alpha \rho_{0}$ (constante restrita), o modelo de correção de erros será

$$
\Delta Z_{t}=\alpha\left(\beta^{\prime} Z_{t-1}+\rho_{0}\right)+\Gamma_{1} \Delta Z_{t-1}+\cdots+\Gamma_{\mathrm{p}-1} \Delta Z_{t-p+1}+\varepsilon_{\mathrm{t}}
$$

e todas as series em $Z_{t}$ são I(1) sem "drift" e as relações de cointegração $\beta^{\prime} Z_{t}$ possuem média $\rho_{0}$.

- Caso $\mu_{\mathrm{t}}=\mu_{0}$ (constante irrestrita), o modelo de correção de erros será

$$
\Delta Z_{t}=\mu_{0}+\alpha \beta^{\prime} Z_{t-1}+\Gamma_{1} \Delta Z_{t-1}+\cdots+\Gamma_{\mathrm{p}-1} \Delta Z_{t-p+1}+\varepsilon_{\mathrm{t}}
$$

e todas as series em $Z_{t}$ são I(1) com "drift" e as relações de cointegração $\beta^{\prime} Z_{t}$ possuem média diferente de zero.

- Caso $\mu_{\mathrm{t}}=\mu_{0}+\alpha \rho_{1} \mathrm{t}$ (tendência restrita), o modelo de correção de erros será

$$
\Delta Z_{t}=\mu_{0}+\alpha\left(\beta^{\prime} Z_{t-1}+\rho_{1} \mathrm{t}\right)+\Gamma_{1} \Delta Z_{t-1}+\cdots+\Gamma_{\mathrm{p}-1} \Delta Z_{t-p+1}+\varepsilon_{\mathrm{t}}
$$

e todas as séries em $Z_{t}$ são I(1) com "drift" $\mu_{0}$ e as relações de cointegração $\beta^{\prime} Z_{t}$ possuem termo de tendência linear $\rho_{1}$ t. 
- Caso $\mu_{\mathrm{t}}=\mu_{0}+\mu_{1} \mathrm{t}$ (tendência restrita) o modelo de correção de erros será

$$
\Delta Z_{t}=\mu_{0}+\mu_{1} \mathrm{t}+\alpha \beta^{\prime} Z_{t-1}+\Gamma_{1} \Delta Z_{t-1}+\cdots+\Gamma_{\mathrm{p}-1} \Delta Z_{t-p+1}+\varepsilon_{\mathrm{t}}
$$

e todas as series em $Z_{t}$ são $\mathrm{I}(1)$ com tendência linear e as relações de cointegração $\beta^{\prime} Z_{t}$ possuem tendência linear.

Como o posto da matriz $\Pi$ fornece o número de relações de cointegração em $Z_{t}$, a estatística razão de verossimilhança (Likelihood Ratio - LR) para determinar o número de relações de cointegração foi formulado para determinar o posto de П. Estes testes são baseados nos autovalores estimados $\hat{\lambda}_{1}, \hat{\lambda}_{2}, \ldots, \hat{\lambda}_{\mathrm{n}}$ da matriz $\widehat{\Pi}$, pois o posto de $\Pi$ é equivalente ao número de autovalores não nulos da matriz.

$\mathrm{O}$ teste LR de Johansen testa as hipóteses $\mathrm{H}_{0}: r=\mathrm{r}_{0}$ vs $\mathrm{H}_{1}: \mathrm{r}>\mathrm{r}_{0}$ através da estatística chamada traço dada por

$$
\operatorname{LR}_{\text {traço }}\left(\mathrm{r}_{0}\right)=-T \sum_{i=\mathrm{r}_{0}+1}^{n} \ln \left(1+\hat{\lambda}_{\mathrm{i}}\right)
$$

Se o posto de $\Pi$ for $\mathrm{r}_{0}$ então $\hat{\lambda}_{\mathrm{r}_{0}+1}, \ldots, \hat{\lambda}_{\mathrm{n}}$ são todos próximos de zero e $L R_{\text {traço }}\left(\mathrm{r}_{0}\right)$ tende a ser pequeno. Por outro lado, se o posto de $\Pi$ for maior que $r_{0}$, alguns dos autovalores $\hat{\lambda}_{\mathrm{r}_{0}+1}, \ldots, \hat{\lambda}_{\mathrm{n}}$ serão não nulos e $L R_{\text {traço }}\left(\mathrm{r}_{0}\right)$ tende a ser maior. A distribuição de $L R_{\text {traço }}\left(\mathrm{r}_{0}\right)$ é uma versão multivariada da distribuição de raiz unitária de Dickey-Fuller que depende da dimensão de $n-\mathrm{r}_{0}$ e da especificação dos termos determinísticos. Os valores críticos desta distribuição foram tabulados por Osterwald-Lenum (1992) para os 5 casos de tendências apresentados.

Estimado o posto $r$ de $\Pi$, Johansen (1988) mostra que o estimador de máxima verossimilhança de $\beta$ é dado por $\widehat{\beta}_{\mathrm{MV}}=\left(\hat{v}_{1}, \hat{v}_{2}, \ldots, \hat{v}_{\mathrm{r}}\right)$ em que $\hat{v}_{\mathrm{i}}$ são os autovetores associados aos autovalores $\hat{\lambda}_{\mathrm{i}}$ e os estimadores de máxima verossimilhança dos demais parâmetros são obtidos pela regressão multivariada substituindo-se $\beta$ por $\widehat{\beta}_{\mathrm{MV}}$. 


\subsection{Simulação}

Nesta seção será avaliado o poder dos testes de cointegração e a precisão dos estimadores do parâmetro de cointegração $\beta$. Para tanto 500 simulações de diferentes configurações do modelo de correção de erros (4.1) foram efetuadas com tamanho amostral 500, utilizando ruídos $\varepsilon_{t} \sim N(0,0.5)$. A tabela abaixo apresenta as configurações utilizadas:

Tabela 4.3 - tabela de parâmetros para a simulação de séries cointegradas

\begin{tabular}{|c|c|c|c|c|c|c|c|}
\cline { 2 - 8 } \multicolumn{1}{c|}{} & $\boldsymbol{\alpha 1}$ & $\boldsymbol{\alpha 2}$ & $\boldsymbol{\beta 2}$ & $\mathbf{a 1 1}$ & $\mathbf{a 1 2}$ & $\mathbf{a 2 1}$ & $\mathbf{a 2 2}$ \\
\hline $\mathbf{1}$ & 0 & 0 & 0 & 0 & 0 & 0 & 0 \\
\hline $\mathbf{2}$ & 0 & 0 & 0 & 0.5 & 0.5 & 0.5 & 0.5 \\
\hline $\mathbf{3}$ & 0.1 & 0 & 0.1 & 0 & 0 & 0 & 0 \\
\hline $\mathbf{4}$ & 0.1 & 0 & 0.1 & 0.5 & 0.5 & 0.5 & 0.5 \\
\hline $\mathbf{5}$ & 0.1 & 0 & 0.8 & 0 & 0 & 0 & 0 \\
\hline $\mathbf{6}$ & 0.1 & 0 & 0.8 & 0.5 & 0.5 & 0.5 & 0.5 \\
\hline $\mathbf{7}$ & 0.8 & 0 & 0.1 & 0 & 0 & 0 & 0 \\
\hline $\mathbf{8}$ & 0.8 & 0 & 0.1 & 0.5 & 0.5 & 0.5 & 0.5 \\
\hline $\mathbf{9}$ & 0.8 & 0 & 0.8 & 0 & 0 & 0 & 0 \\
\hline $\mathbf{1 0}$ & 0.8 & 0 & 0.8 & 0.5 & 0.5 & 0.5 & 0.5 \\
\hline
\end{tabular}

Foram utilizados os três procedimentos: Engle e Granger (1986) - OLS, Stock e Watson (1993) - DOLS e Johansen (1988), com nível de significância do teste de 5\% e 1\%.

Para cada um dos modelos simulados foram observadas as quantidades de testes que aceitaram e rejeitaram a hipótese nula de não cointegração dentro dos 500 testes efetuados. Como nos modelos 1 e 2 os parâmetros $\alpha_{1}, \alpha_{2}$ e $\beta_{2}$ são nulos as séries simuladas são não cointegradas. Para estes modelos foram calculadas as quantidades de testes que rejeitam a hipótese nula tendo-se, portanto, uma estimativa do erro de tipo I, ou seja, rejeitar $\mathrm{H}_{0}$ sendo ela verdadeira. Para os modelos restantes, cointegrados, foram observadas as quantidades de aceitação da hipótese nula dentre todos os testes tendo-se assim, uma estimativa do erro de tipo II, ou seja, aceitar $\mathrm{H}_{0}$ sendo esta falsa.

A tabela abaixo apresenta os resultados obtidos para a realização dos testes sobre as 500 simulações dos diferentes modelos. Para os modelos 1 e 2 a tabela apresenta os valores de erro de tipo I e para os demais testes os valores apresentados correspondem ao erro de tipo II. 
Tabela 4.4 - Resultados dos testes de cointegração

\begin{tabular}{|c|c|c|c|c|c|c|}
\cline { 2 - 7 } \multicolumn{1}{c|}{} & \multicolumn{2}{c|}{ OLS } & \multicolumn{2}{c|}{ DOLS } & \multicolumn{2}{c|}{ Johansen } \\
\hline Modelo & $\mathbf{5 . 0 0 \%}$ & $\mathbf{1 . 0 0 \%}$ & $\mathbf{5 . 0 0 \%}$ & $\mathbf{1 . 0 0 \%}$ & $\mathbf{5 . 0 0 \%}$ & $\mathbf{1 . 0 0 \%}$ \\
\hline $\mathbf{1}$ & $3.60 \%$ & $1.00 \%$ & $4.80 \%$ & $0.60 \%$ & $6.40 \%$ & $1.80 \%$ \\
\hline $\mathbf{2}$ & $5.20 \%$ & $1.20 \%$ & $1.00 \%$ & $0.40 \%$ & $16.60 \%$ & $5.40 \%$ \\
\hline $\mathbf{3}$ & $0.00 \%$ & $0.20 \%$ & $0.00 \%$ & $0.20 \%$ & $0.20 \%$ & $8.00 \%$ \\
\hline $\mathbf{4}$ & $100.00 \%$ & $100.00 \%$ & $0.00 \%$ & $0.00 \%$ & $0.00 \%$ & $0.00 \%$ \\
\hline $\mathbf{5}$ & $0.00 \%$ & $0.00 \%$ & $0.00 \%$ & $0.00 \%$ & $0.00 \%$ & $0.40 \%$ \\
\hline $\mathbf{6}$ & $18.40 \%$ & $69.60 \%$ & $0.00 \%$ & $0.00 \%$ & $0.00 \%$ & $0.00 \%$ \\
\hline $\mathbf{7}$ & $0.00 \%$ & $0.00 \%$ & $0.00 \%$ & $0.00 \%$ & $0.00 \%$ & $0.00 \%$ \\
\hline $\mathbf{8}$ & $0.00 \%$ & $0.00 \%$ & $0.00 \%$ & $0.00 \%$ & $0.00 \%$ & $0.00 \%$ \\
\hline $\mathbf{9}$ & $0.00 \%$ & $0.00 \%$ & $0.00 \%$ & $0.00 \%$ & $0.00 \%$ & $0.00 \%$ \\
\hline $\mathbf{1 0}$ & $0.00 \%$ & $0.00 \%$ & $0.00 \%$ & $0.00 \%$ & $0.00 \%$ & $0.00 \%$ \\
\hline
\end{tabular}

Observa-se que os valores obtidos de erro de tipo I para o modelo 1, como esperado, se aproximam dos valores de nível dos testes. No entanto, entre os valores obtidos para o modelo 2 apenas aqueles do teste OLS mantiveram-se próximos dos valores de nível dos testes, sendo que o teste de Johansen apresentou valorese maiores e o teste DOLS gerou valores menores de erro do tipo I.

Observa-se que tanto o método DOLS quanto o de Johansen possuem baixos valores de erro de tipo II para todos os modelos simulados e o teste OLS obteve altos níveis de erro de tipo II para os modelos 4 e 6 indicando que, para baixos valores de $\alpha_{1}$ e $\beta_{2}$, o resultado pode ser contaminado pela presença de fatores auto-regressivos nas séries. Com base nestes resultados pode-se afirmar que os testes DOLS e de Johansen são testes mais poderosos do que o método OLS.

Novamente, a comparação dos estimadores de diferentes métodos será efetuada através das medidas de média, erro quadrático médio e dispersão das estimativas calculadas nas simulações. A tabela abaixo apresenta os resultados encontrados. Nesta tabela não estão presentes os modelos 1 e 2 por se tratarem de modelos não cointegrados e, portanto, possuem valores $\beta_{2}=0$. 
Tabela 4.5 - Comparação entre os estimadores do vetor de cointegração

\begin{tabular}{|c|c|c|c|c|c|}
\hline Modelo & beta & Estatística & OLS & DOLS & Johansen \\
\hline \multirow{2}{*}{3} & \multirow{8}{*}{0.1} & media & 0.087 & 0.092 & 0.103 \\
\hline & & EQM & 2.008 & 2.127 & 2.712 \\
\hline \multirow{2}{*}{4} & & media & 0.335 & 0.187 & 0.100 \\
\hline & & EQM & 35.590 & 5.935 & 0.451 \\
\hline \multirow{2}{*}{7} & & media & 0.099 & 0.100 & 0.101 \\
\hline & & EQM & 0.034 & 0.037 & 0.045 \\
\hline \multirow{2}{*}{8} & & media & 0.107 & 0.099 & 0.100 \\
\hline & & EQM & 0.040 & 0.008 & 0.006 \\
\hline \multirow{2}{*}{5} & \multirow{8}{*}{0.8} & media & 0.726 & 0.750 & 0.806 \\
\hline & & EQM & 5.841 & 3.976 & 3.008 \\
\hline \multirow{2}{*}{6} & & media & 0.817 & 0.811 & 0.800 \\
\hline & & EQM & 0.239 & 0.113 & 0.024 \\
\hline \multirow{2}{*}{9} & & media & 0.790 & 0.800 & 0.800 \\
\hline & & EQM & 0.117 & 0.037 & 0.036 \\
\hline \multirow{2}{*}{10} & & media & 0.801 & 0.800 & 0.800 \\
\hline & & EQM & 0.002 & 0.000 & 0.000 \\
\hline
\end{tabular}

Observa-se que, em média, todos os estimadores se aproximam do valor verdadeiro do coeficiente de cointegração, sendo que os métodos DOLS e de Johansen são os mais próximos.

No caso das estimativas geradas pelo métodos OLS e DOLS para o modelo 4, nota-se uma grande perturbação ocasionada pela presença de coeficientes auto-regressivos e baixos valores de $\alpha_{1}$ e $\beta_{2}$.

O método de Johansen foi o que apresentou menor erro quadrático médio para todos os modelos simulados, com excessão dos modelos 3 e 7 em que os métodos OLS e DOLS tiveram uma melhor performance. Comparando os modelos 3 e $4\left(\alpha_{1}=0,1\right)$ com os modelos 7 e 8 $\left(\alpha_{1}=0,8\right)$ nota-se que o EQM diminui com o aumento do parâmetro de velocidade. Esses resultados se repetem na comparação dos modelos 5 e 6 com os modelos 9 e 10, indicando que baixos valores de $\alpha_{1}$ comprometem a precisão do estimador do parâmetro de cointegração.

Os gráficos boxplot abaixo apresentam a dispersão das estimativas encontradas para os parâmetros de cointegração dos diferentes modelos, agrupadas pelos diferentes valores de $\beta_{2}(0,1$ e 0,8$)$. 


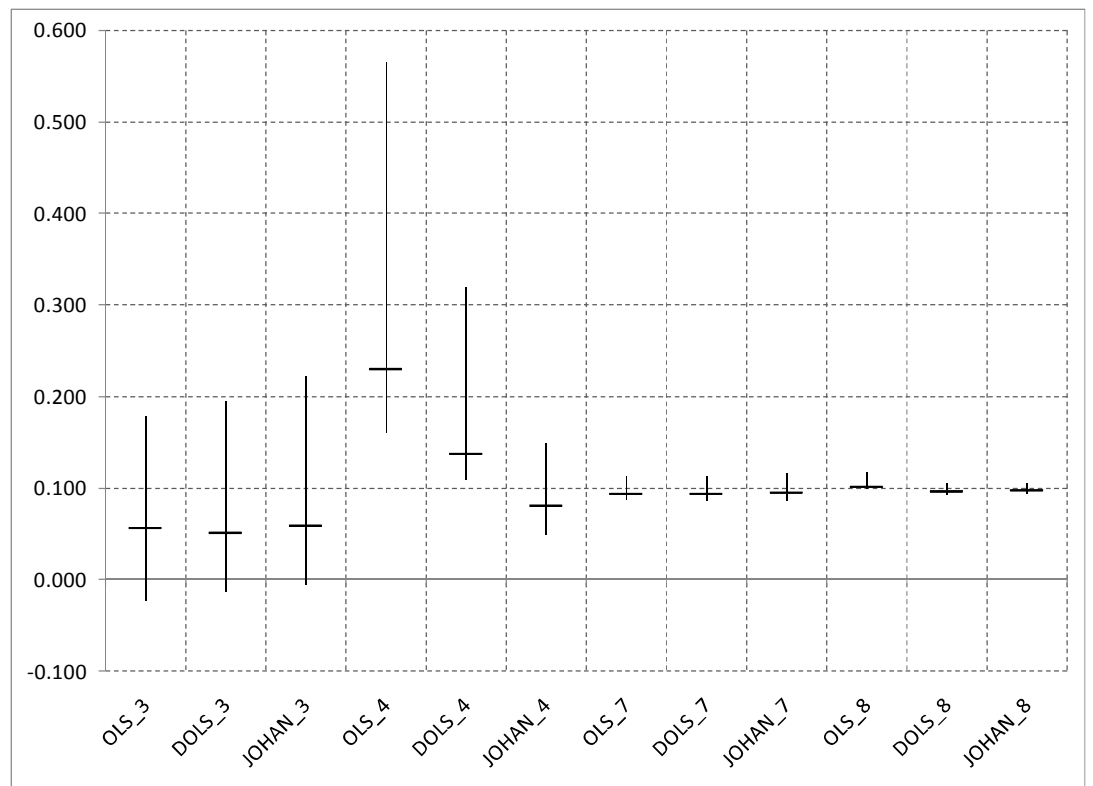

(a) $\boldsymbol{\beta}_{2}=\mathbf{0 , 1}$

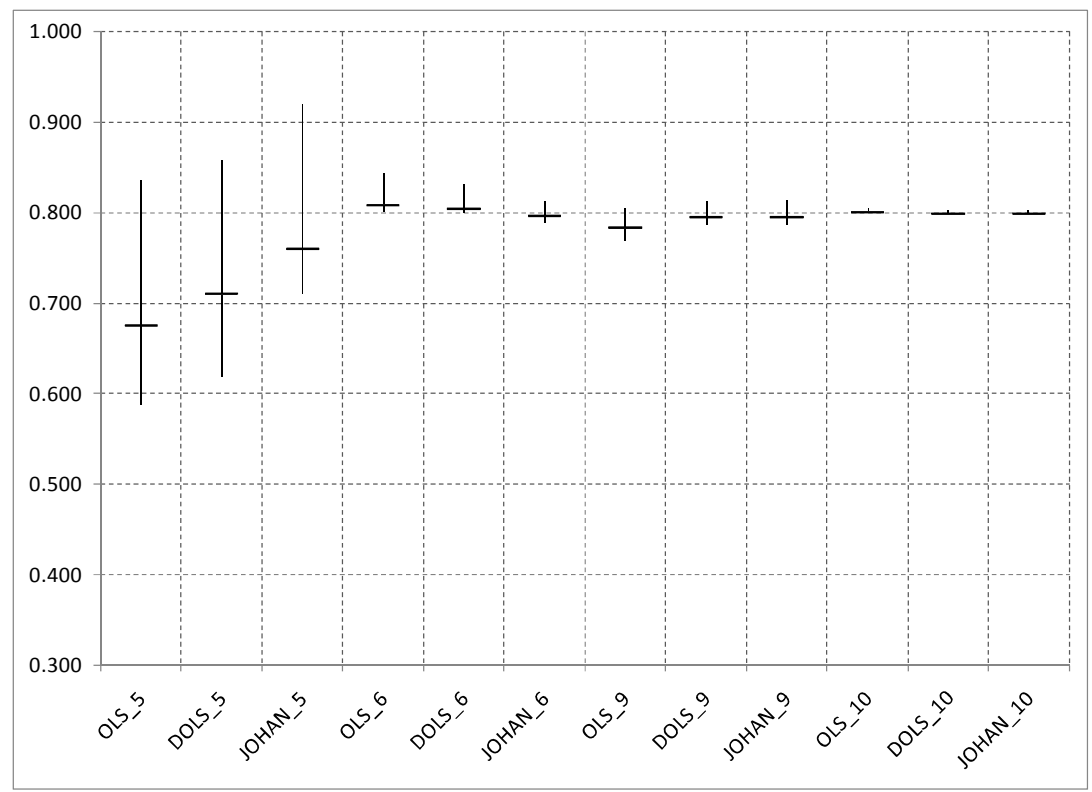

(b) $\boldsymbol{\beta}_{2}=\mathbf{0 , 8}$

Figura 4.3 - Dispersão das estimativas do parâmetro de cointegração

Por estes gráficos, pode-se comprovar o efeito dos baixos valores de $\alpha_{1}$ aumentando a dispersão das estimativas e o desvio nas estimativas para os métodos OLS e DOLS no caso do modelo 4. Para os demais modelos todos os métodos apresentaram bons resultados, com 
excessão do método OLS para o modelo 9 que apresentou um pequeno desvio do valor real de $\beta_{2}=0,8$. 


\section{Capítulo 5 Cointegração Fracionária}

Como apresentado, os métodos de cointegração são utilizados para se determinar as relações entre séries não estacionárias. No entanto, o fato de assumir que o parâmetro auto-regressivo é unitário tem sido criticado e um método alternativo seria a utilização de processos fracionários como apresentado por Cheung e Lai (1993), Baillie e Bollerslev (1994), Robinson e Marinucci (1998). O gráfico abaixo apresenta duas séries cointegradas fracionárias.

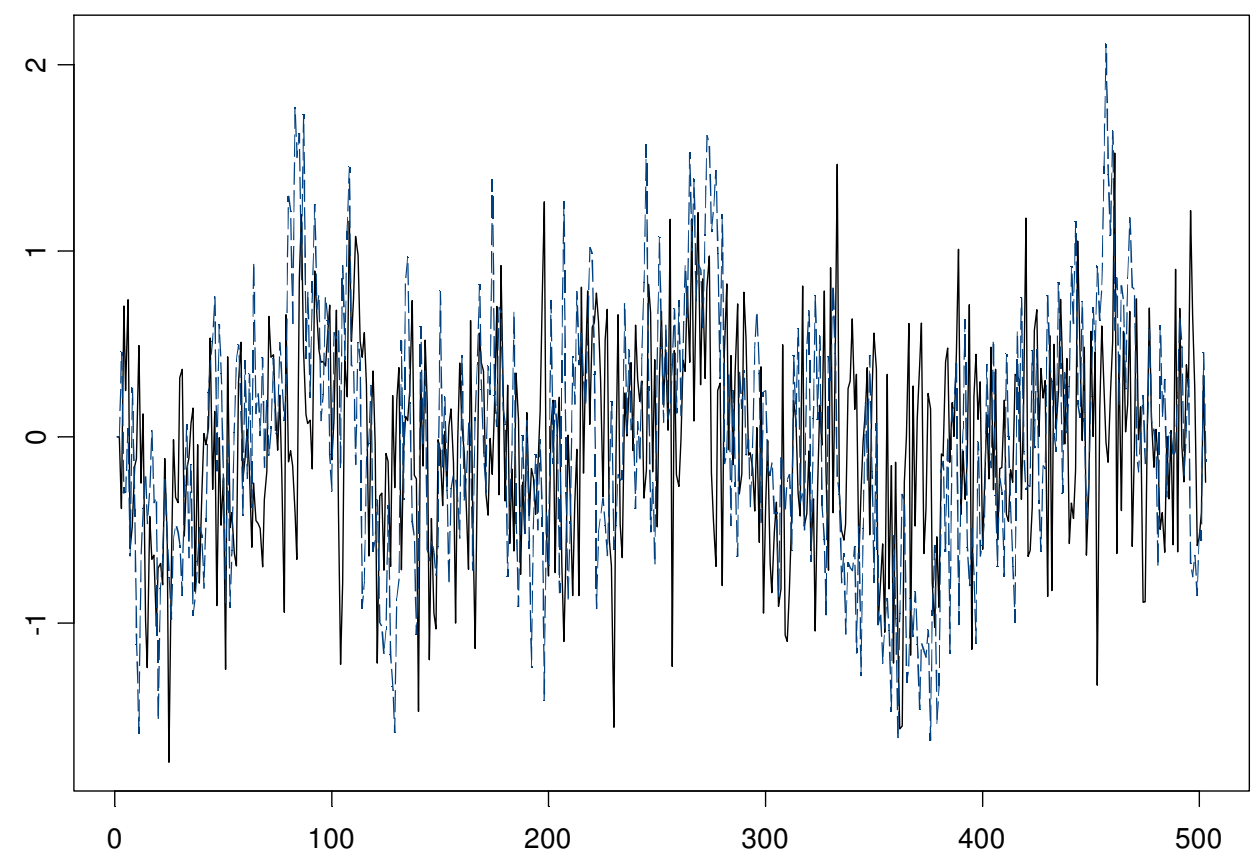

Figura 5.1 - Exemplo de séries cointegradas fracionárias

Assim como no caso de séries não estacionárias, séries fracionárias também apresentam o problema de regressão espúria, como analisado por Marmol (1998).

Considere os seguintes processos fracionários independentes e não cointegrados

$$
\begin{aligned}
& \Delta^{0.45} z_{1 t}=u_{1 t}, \\
& \Delta^{0.45} z_{2 t}=u_{2 t},
\end{aligned}
$$


sendo $u_{t} \sim \operatorname{RBN}(0,1)$ a simulação de 500 observações pode ser observada no gráfico abaixo.

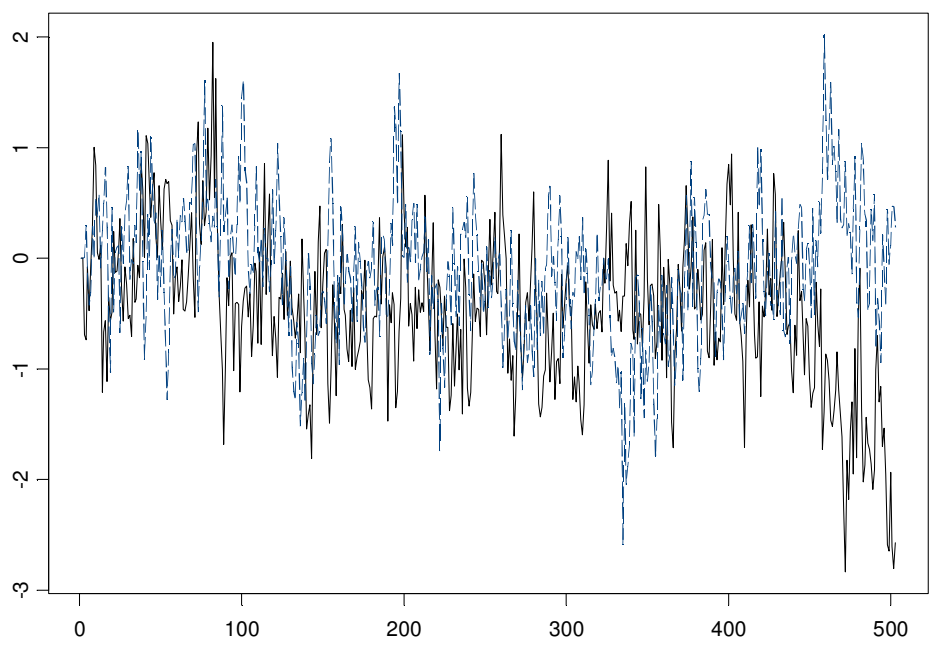

Figura 5.2 - Duas series fracionárias e não cointegradas

A regressão entre estas séries apresenta os seguintes resultados:

Tabela 5.1 - Regressão entre duas series fracionárias

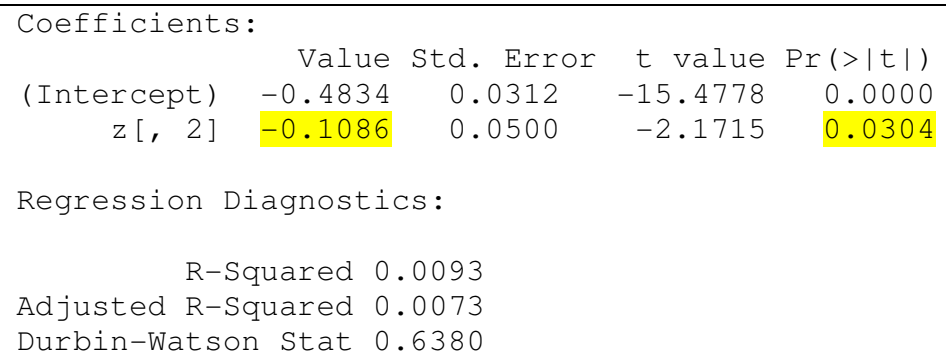

A estimativa $0,1086(\mathrm{p}-$ valor $=0,0304)$ é significante. Porém, tomando-se a diferença das séries observamos a verdadeira relação entre as mesmas $(p-$ valor $>0,05)$. 
Tabela 5.2 - Regressão entre duas series em diferenças fracionárias

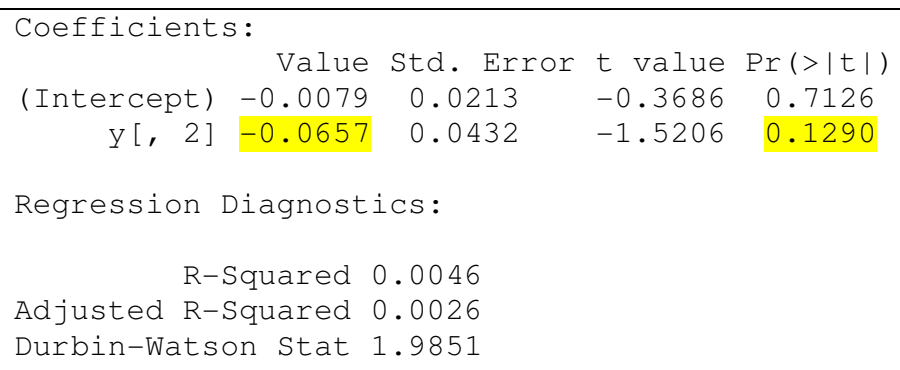

Observa-se que são necessárias diferentes formas de tratamento para se encontrar as verdadeiras relações entre séries fracionária, da mesma forma que para as séries não estacionárias, fazendo sentido portanto a definição de cointegração fracionária.

Seguindo os princípios introduzidos por Engle e Granger (1987), apresentados na seção 4.1, e desenvolvidos por Caporale e Gil-Alana (2004), um conjunto de $n$ séries temporais $Z_{t}(n \times 1)$ é chamado cointegrado fracionário de ordem $(d, b)-C I(d, b), d, b \in \mathbb{R}^{+}$, se todos os processos forem integrados de ordem $d-I(d)$ e se existir um vetor $\beta(n \times 1)$ tal que $\beta^{\prime} \times Z_{t}$ seja uma série de ordem $b-I(b)$ para $0<b<d$.

\subsection{Modelo de Correção de Erros}

O modelo de correção de erros pode ser generalizado da seguinte forma:

$$
\left\{\Phi(\mathrm{B})+\left[(1-\mathrm{B})^{-\mathrm{b}}-1\right] \alpha \beta^{\prime}\right\}(1-\mathrm{B})^{\mathrm{d}} Z_{t}=\Theta(\mathrm{B}) \varepsilon_{\mathrm{t}}
$$

em que $Z_{t}$ é o vetor de séries temporais e $\varepsilon_{\mathrm{t}} \sim i . i . d .(0, \Sigma)$, ambos $(N \times 1), \Phi(\mathrm{B})$ e $\Theta(\mathrm{B})$ são matrizes polinomiais de ordem finita com todas as raízes características fora do círculo unitário e as matrizes $(N \times r)$ constantes $\alpha$ e $\beta$, que possuem posto $r$, representam, respectivamente, o coeficiente de correção de erros e coeficiente de cointegração.

Pode-se notar que para o balanceamento da equação a seguinte condição é necessária

$$
(1-\mathrm{B})^{\mathrm{d}-\mathrm{b}} \beta^{\prime} Z_{t} \sim I(0)
$$

Este modelo admite que todas as séries possuem o mesmo nível de integração o que pode não ser verdade. Davidson (2002) sugere as seguintes generalizações: 


$$
\Delta(\mathrm{B})=\left[\begin{array}{ccc}
(1-\mathrm{B})^{\mathrm{d}_{1}} & \cdots & 0 \\
\vdots & \ddots & \vdots \\
0 & \cdots & (1-\mathrm{B})^{\mathrm{d}_{n}}
\end{array}\right] \text { e K(B)}=\left[\begin{array}{ccc}
(1-\mathrm{B})^{\mathrm{b}_{1}} & \cdots & 0 \\
\vdots & \ddots & \vdots \\
0 & \cdots & (1-\mathrm{B})^{\mathrm{b}_{n}}
\end{array}\right]
$$

de forma que o modelo de correção de erros passa a ser

$$
\left\{\Phi(\mathrm{B})+\left[\mathrm{K}(\mathrm{B})^{-1}-I\right] \alpha \beta^{\prime}\right\} \Delta(\mathrm{B}) Z_{t}=\Theta(\mathrm{B}) \varepsilon_{\mathrm{t}}
$$

e a condição para o balanceamento alterada, admitindo-se $w_{t}=\Delta(\mathrm{B}) Z_{t} \sim I(0)$,

$$
\beta^{\prime} \mathrm{K}(\mathrm{B})^{-1} w_{t} \sim I(0)
$$

Este modelo é chamado modelo vetorial fracionário de correção de erros (Fractional Vector Error Correction Model - FVECM).

O FVECM é um modelo bastante geral e o caso mais próximo do VECM seria quando $\mathrm{d}_{\mathrm{i}}-\mathrm{b}_{\mathrm{i}}=a \geq 0$ para todos os valores de $\mathrm{i}$, tal que $\mathrm{K}(\mathrm{B})^{-1} \Delta(\mathrm{B})=(1-\mathrm{B})^{a} I$.

\subsection{Testes para Cointegração Fracionária}

Davidson (2002) expôs, em sua pesquisa, que, no escopo da cointegração fracionária, as implicações do teorema central do limite não são válidas quando as ordens de integração não são conhecidas e as estatísticas de teste não são assintoticamente pivotais, sendo dependentes do parâmetro de integração fracionária e dos parâmetros relacionados à covariância, dependendo, portanto, da convergência de funcionalidades do movimento browniano fracionário, que possui incrementos correlacionados, invalidando as tabulações dos testes padrões.

A solução proposta pelo autor é a utilização de simulações por bootstrap, que geram aproximações assintóticas de primeira ordem para testes não pivotais, visando gerar a distribuição empírica das estatíticas de inferência sobre a hipótese de cointegração. Três hipóteses são, então, avaliadas: simples cointegração (hipótese fraca), cointegração e causalidade de Granger (hipótese semifraca) e independência estatística (hipótese forte).

Quando $\alpha=\beta=0$ em (5.1) pode-se afirmar que as séries não são cointegradas e o modelo passa a ser

$$
\Phi(\mathrm{B}) \Delta(\mathrm{B}) Z_{t}=\Theta(\mathrm{B}) \varepsilon_{\mathrm{t}}
$$


O procedimento proposto por Davidson (2002) assumindo $\mathrm{H}_{0}: \mathrm{K}(\mathrm{B})=I$ (não cointegração) possui as seguintes etapas:

1. Processos $\operatorname{ARFIMA}(p, d, q)$ são ajustados às séries temporais;

2. A diferenciação fracionária é aplicada às séries de acordo com o modelo ajustado no passo 1, para se estimar $w_{t}$ em (5.2) e então ajustar modelos dinâmicos. O objetivo é estimar o modelo (5.1) condicionado às restrições da hipótese nula de não cointegração;

3. Os resíduos do passo $2\left(\varepsilon_{\mathrm{t}}\right)$ são reamostrados $\mathrm{M}$ vezes com reposição e utilizados para gerar as séries $\left\{\mathrm{Z}_{\mathrm{t}}^{(\mathrm{i})}\right\}_{\mathrm{i}=1}^{\mathrm{M}}$ de acordo com (5.1), sob $\mathrm{H}_{0}$, que preservam a mesma dinâmica de $Z_{t}$, mas que são, por construção, não cointegradas. As distribuições dos testes estatísticos são, então, estimadas utilizando $\left\{\mathrm{Z}_{\mathrm{t}}^{(\mathrm{i})}\right\}_{\mathrm{i}=1}^{\mathrm{M}}$.

Obtidas as distribuições de bootstrap das estatísticas das séries não cointegradas, porém com a mesma dinâmica das séries reais, são calculadas as estatísticas das séries reais cujos valores são localizados nas distribuições para se obter p-valores estimados, que, de acordo com o autor, são aproximações válidas de primeira ordem dos p-valores verdadeiros.

Para a realização dos testes de estatísticas baseadas em regressão é considerada a partição $Z_{t}=\left(x_{t}, Y_{t}^{\prime}\right)^{\prime}$ em que é necessária apenas a reamostragem de $x_{t}$.

Este procedimento testa a independência estatística entre as séries, ou hipótese forte como nomeado pelo autor. Para que a presença das relações entre as séries sejam conservadas na série simulada pelo bootstrap ao invés de se reamostrar a série $\widehat{\varepsilon}_{\mathrm{t}}^{\mathrm{x}}$ (resíduo obtido após o ajuste do modelo ARFIMA à série $x_{t}$ ), no terceiro passo, são geradas reamostragens de $\hat{u}_{t}$, obtida através da regressão de $\widehat{\varepsilon}_{\mathrm{t}}^{\mathrm{x}}$ por $\widehat{\varepsilon}_{\mathrm{t}-1}^{\mathrm{x}}, \widehat{\varepsilon}_{\mathrm{t}+1}^{\mathrm{y}}, \widehat{\varepsilon}_{\mathrm{t}}^{\mathrm{y}}$ e $\widehat{\varepsilon}_{\mathrm{t}-1}^{\mathrm{y}}$, em que $\widehat{\varepsilon}_{\mathrm{t}}^{\mathrm{y}}$ são os resíduos obtidos após os ajustes dos modelos ARFIMA às séries $Y_{t}$. As reamostragens de $\hat{u}_{t}$ são utilizadas para se obter $\left\{\widehat{\varepsilon}_{\mathrm{t}}^{\mathrm{x}(\mathrm{i})}\right\}_{\mathrm{i}=1}^{\mathrm{M}}$, através da equação obtida pela regressão, que é usada para gerar $\left\{X_{t}^{(i)}\right\}_{i=1}^{M}$ e efetuar os testes de não cointegração, ou hipótese fraca. 
Para os testes de hipótese semifraca, ou de cointegração e causalidade de Granger, na regressão mencionada acima são suprimidas as séries $\widehat{\varepsilon}_{\mathrm{t}+1}^{\mathrm{y}}$.

São indicados dois testes residuais para a determinação da presença de cointegração fracionária nas séries: Engle e Granger Fracionário (nomenclatura utilizada por Marques (2007)) e Durbin-Watson, que são efetuados sobre os resíduos $\delta_{t}$ da regressão de $x_{t}$ e $Y_{t}$.

\section{Engle e Granger Fracionário (FEG)}

Este teste é realizado através da estimação do parâmetro de memória longa dos resíduos da regressão da série simulada com as séries observadas, semelhante ao teste de Engle e Granger (1986).

Durbin-Watson (DW)

$$
\mathrm{DW}=-2 \frac{\sum_{\mathrm{t}=2}^{\mathrm{N}} \hat{\delta}_{t-1} \Delta \hat{\delta}_{t}}{\sum_{\mathrm{t}=2}^{\mathrm{N}} \hat{\delta}_{\mathrm{t}-1}^{2}}
$$

\subsection{Simulação}

Para avaliação do poder destes testes de cointegração fracionária, foram simulados modelos idênticos ao modelos de correção de erros fracionários (5.1) utilizando os seguintes parâmetros:

Tabela 5.3 - Parâmetros utilizados na simulação de cointegração fracionária

\begin{tabular}{|c|c|c|c|c|c|}
\hline Modelo & $\mathbf{d}$ & $\boldsymbol{\alpha 1}$ & $\boldsymbol{\alpha 2}$ & $\boldsymbol{\beta} \mathbf{1}$ & $\boldsymbol{\beta 2}$ \\
\hline $\mathbf{1}$ & 0.55 & 0 & 0 & 0 & 0 \\
\hline $\mathbf{2}$ & 0.55 & 0.2 & 0 & 1 & 0.5 \\
\hline $\mathbf{3}$ & 0.55 & 1.0 & 0 & 1 & 0.8 \\
\hline $\mathbf{4}$ & 0.75 & 0 & 0 & 0 & 0 \\
\hline $\mathbf{5}$ & 0.75 & 0.2 & 0 & 1 & 0.5 \\
\hline $\mathbf{6}$ & 0.75 & 1.0 & 0 & 1 & 0.8 \\
\hline $\mathbf{7}$ & 1.00 & 0 & 0 & 0 & 0 \\
\hline $\mathbf{8}$ & 1.00 & 0.2 & 0 & 1 & 0.5 \\
\hline $\mathbf{9}$ & 1.00 & 1.0 & 0 & 1 & 0.8 \\
\hline
\end{tabular}

Adicionalmente $\mathrm{d} 1=\mathrm{d} 2=\mathrm{d}, \alpha=\left(\begin{array}{l}\alpha 1 \\ \alpha 2\end{array}\right), \beta=\left(\begin{array}{l}\beta 1 \\ \beta 2\end{array}\right)$ e $\Phi(\mathrm{B})=\Theta(\mathrm{B})=I$. 
Neste caso, o número de simulações ficou limitado a 50 vezes devido ao elevado custo computacional para a realização dos testes (para a realização de cada 10 simulações e testes de cointegração fracionária observou-se períodos de 12 a 24 horas, dependendo das configurações do computador utilizado). Para cada simulação, os testes aplicados utilizaram 500 reamostragens de Bootstrap.

Seguindo a mesma metodologia das simulações do capítulo anterior, para cada um dos modelos simulados foram observadas as quantidades de testes, cujo nível nominal utilizado foi de 5\%, que aceitaram e rejeitaram a hipótese nula de não cointegração dentro dos 50 testes efetuados. Como nos modelos 1, 4 e 7 os parâmetros $\alpha_{1}, \alpha_{2} \beta_{1}$ e $\beta_{2}$ são nulos as séries simuladas são não cointegradas. Para estes modelos foram calculadas as quantidades de testes que rejeitam a hipótese nula tendo-se, portanto, uma estimativa do erro de tipo I. Para os modelos restantes, cointegrados, foram observadas as quantidades de aceitação da hipótese nula dentre todos os testes tendo-se assim, uma estimativa do erro de tipo II e o poder do teste é, então, definido por $\pi=1-$ erro de tipo II.

A tabela abaixo apresenta os resultados obtidos para a realização dos testes sobre as 50 simulações dos diferentes modelos. Para os modelos 1, 4 e 7 a tabela apresenta os valores de erro de tipo I e para os demais testes os valores apresentados correspondem aos valores de erro de tipo II. 
Observa-se que para as séries não cointegradas, o nível de teste estimado diferiu dos 5\% nominais dos testes sendo os testes do modelo 4 os que mais se aproximaram deste valor.

Os testes aplicados em séries cointegradas mostraram-se poderosos na maioria dos casos, com exceção dos modelos com baixos valores de parâmetro de memória longa e de $\alpha$ e $\beta$ em que os valores de erro de tipo II encontrados foram muito elevados (modelos 2 e 5). Pode ser observado que para séries integradas com altos parâmetros de memória longa os testes são poderosos, não dependendo dos valores de $\alpha$ e $\beta$. 
Efetuando-se a comparação dos estimadores OLS do parâmetro de cointegração fracionária pelas mesmas medidas apresentadas anteriormente de média e EQM, foi construida a seguinte tabela:

Tabela 5.5 - Média e EQM dos estimadores do parâmetro de cointegração fracionária

\begin{tabular}{|c|cc|}
\hline Modelo & \multicolumn{2}{|c|}{$\widehat{\boldsymbol{\beta}}$} \\
\hline \multirow{2}{*}{ d0.55alfa0.2beta0.5 } & Media & 0.09 \\
& EQM & 8.991859 \\
\hline \multirow{2}{*}{ d0.55alfa1.0beta0.8 } & Media & 0.41 \\
& EQM & 8.136165 \\
\hline \multirow{2}{*}{ d0.75alfa0.2beta0.5 } & media & 0.23 \\
& EQM & 4.149273 \\
\hline \multirow{2}{*}{ d0.75alfa1.0beta0.8 } & media & 0.63 \\
& EQM & 1.757773 \\
\hline \multirow{2}{*}{ d1.00alfa0.2beta0.5 } & media & 0.48 \\
& EQM & 0.080582 \\
\hline \multirow{2}{*}{ d1.00alfa1.0beta0.8 } & media & 0.79 \\
& EQM & 0.004801 \\
\hline
\end{tabular}

Nota-se a convergência dos valores estimados para os valores reais apenas no caso de séries com elevadas ordens de integração, sendo que aquelas com parâmetros de memória longa de baixa magnitude apresentaram estimativas distantes dos valores reais e elevados valores de EQM, indicando sua divergência com relação ao valor real, como constatado também pelos gráficos de dispersão das estimativas abaixo. 


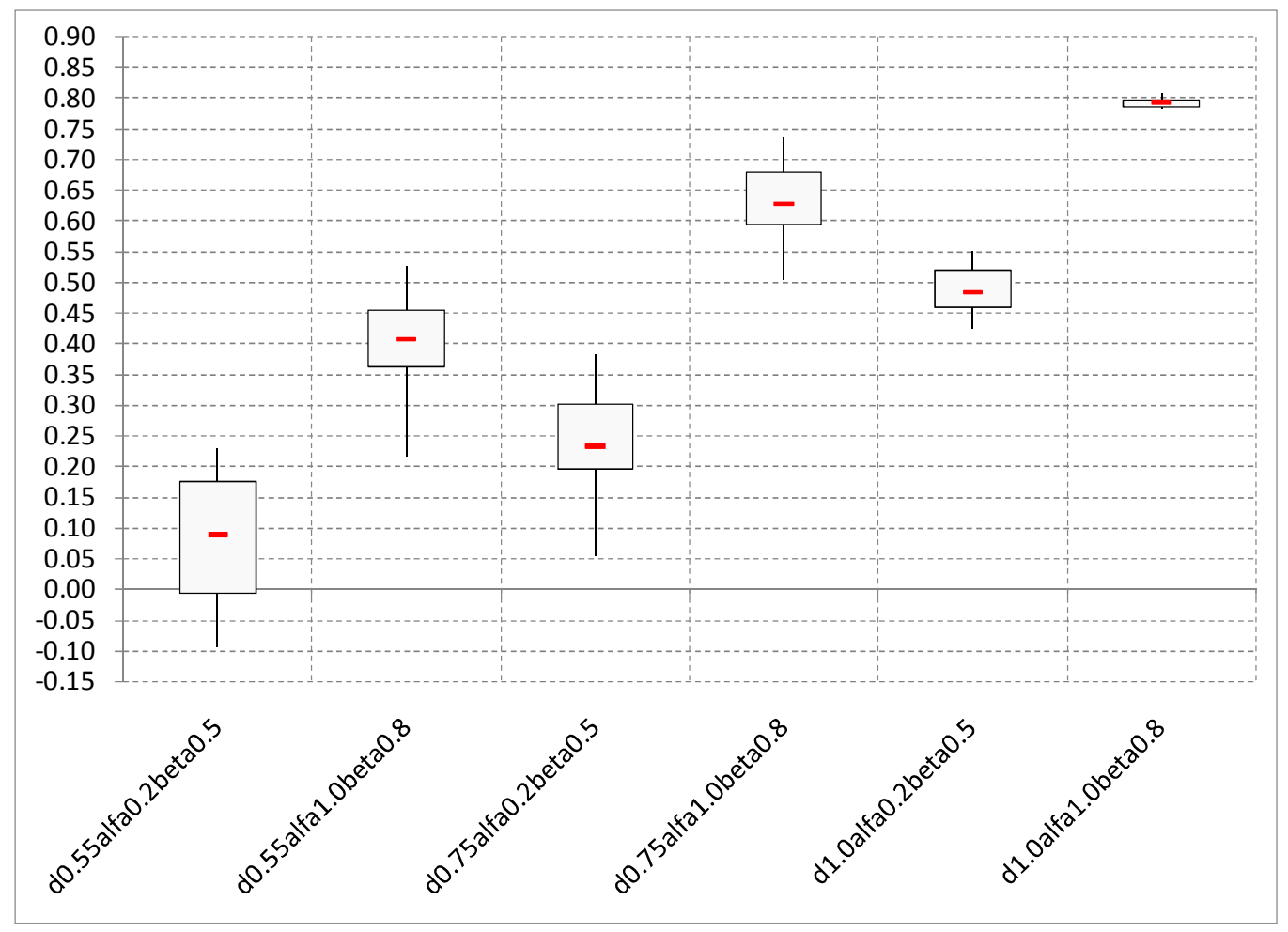

Figura 5.3 - Estimadores do parâmetro de cointegração $\widehat{\beta}$

O gráfico de box plot confirma o fato de melhores estimativas de parâmetro de cointegração serem obtidas na análise de séries com elevados parâmetros de memória longa. 


\section{Capítulo 6 Aplicações em Séries Reais}

Os testes e estimadores apresentados nos capítulos foram aplicados a dados reais com o intuito de demonstrar sua utilização. As séries escolhidas são o preço médio mensal da taxa de câmbio Real/Dólar (preço spot) e sua cotação futura, ou seja, preço futuro de primeiro vencimento (preço futuro). As séries utilizadas são provenientes das negociações diárias na Bolsa de Mercadorias e Futuros BM\&F desde 04/01/1999 a 28/02/2010, totalizando 134 amostras.

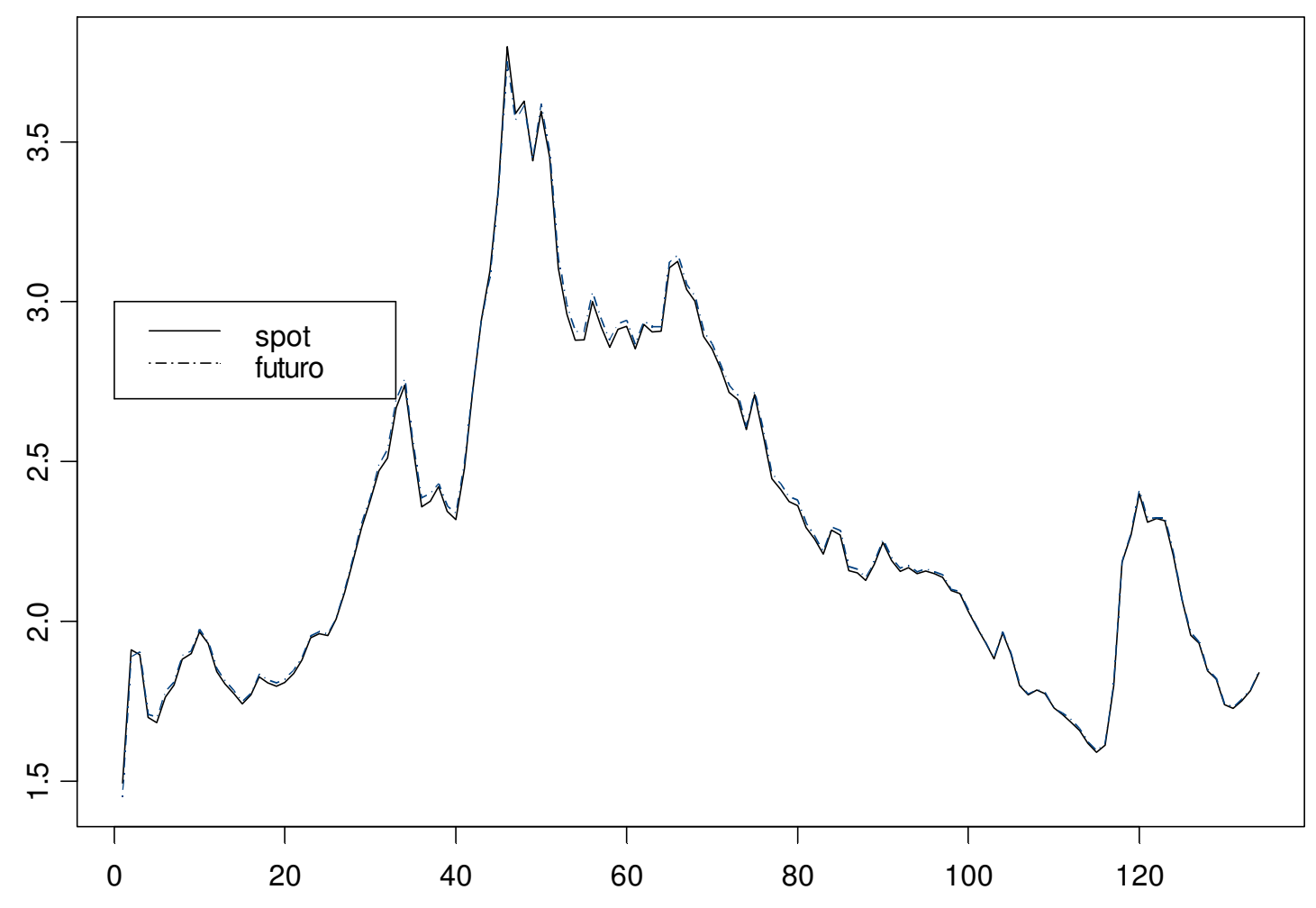

Figura 6.1 - Séries de taxas de câmbio spot e futura

Em finanças, as relações de não arbitragem entre taxas básicas de juros entre países definem que a diferença entre as taxas de câmbio spot e futura é equivalente à diferença entre as taxas de juros interna e externa. Usualmente, preços de taxas de câmbio são definidas como não 
estacionárias e é razoável admitir que a diferença entre as taxas de juros seja estacionário, levando portanto à hipótese de que as taxas de câmbio spot e futura sejam cointegradas com vetor de cointegração $\beta=(1 ;-1)^{\prime}$.

Neste capítulo testes de cointegração e cointegração fracionárias serão realizados e o parâmetro de cointegração estimado. Em seguida, um estudo sobre os resíduos será efetuado para comparação entre os modelos ajustado.

\subsection{Estimação do Modelo de Cointegração Convencional}

Observa-se nos gráficos de FAC (Figura 6.2), tanto do preço spot quanto do preço futuro, um decaimento muito lento indicando a presença de memória longa ou uma raiz unitária.
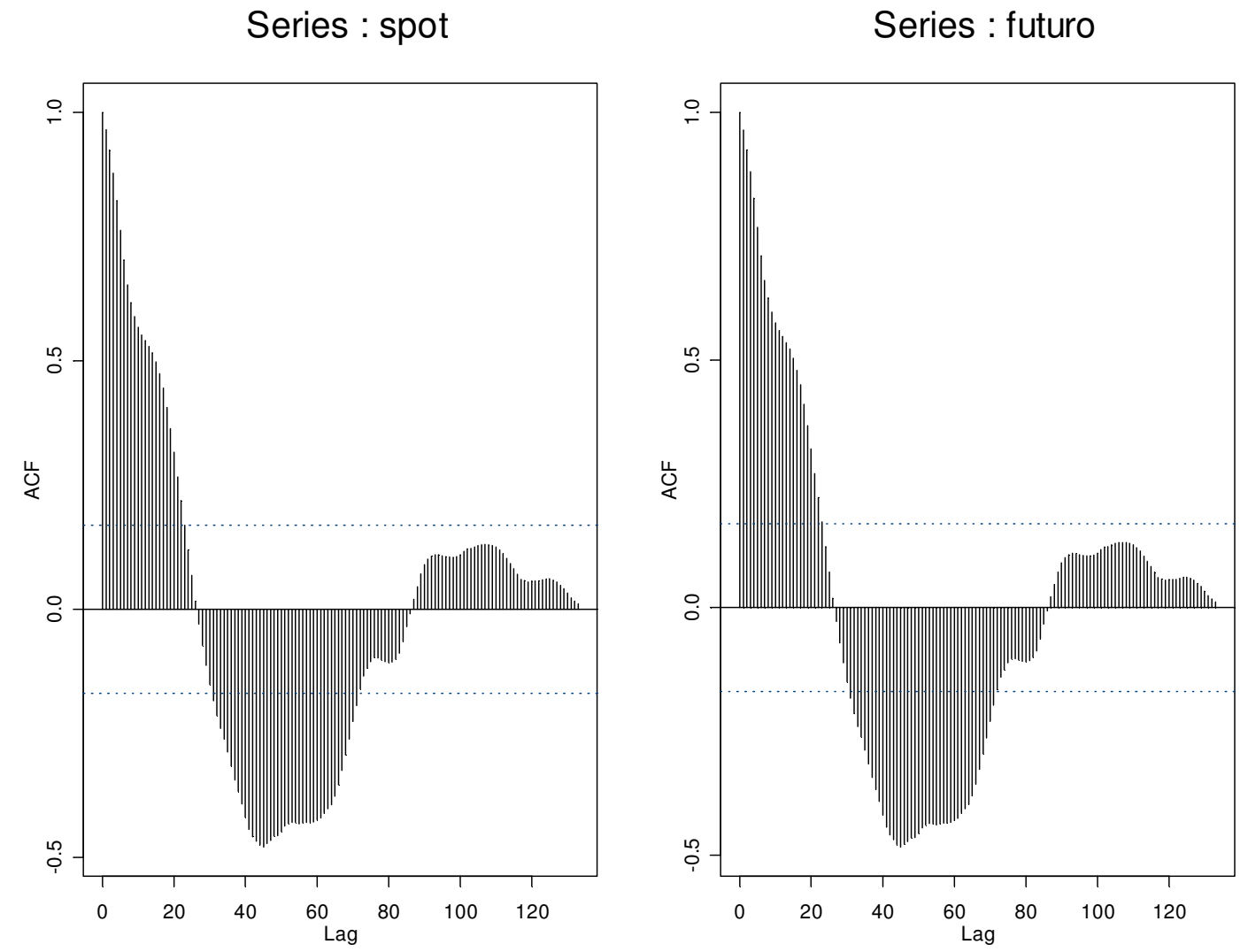

Figura 6.2 - FAC das séries das taxas de câmbio spot e futura

Após a constatação dos gráficos de FAC, testes de raiz unitária foram aplicadas às séries gerando os seguintes resultados: 


\begin{tabular}{|c|c|}
\hline Taxa de Câmbio Spot & Taxa de Câmbio Futura \\
\hline Test for Unit Root: Augmented DF Test & Test for Unit Root: Augmented DF Test \\
\hline $\begin{array}{l}\text { Null Hypothesis: there is a unit root } \\
\text { Type of Test: t-test } \\
\text { Test Statistic: }-1.576 \\
\text { P-value: } 0.4921\end{array}$ & $\begin{array}{l}\text { Null Hypothesis: there is a unit root } \\
\text { Type of Test: t-test } \\
\text { Test Statistic: }-1.623 \\
\text { P-value: } 0.4683\end{array}$ \\
\hline $\begin{array}{l}\text { Coefficients: } \\
\quad \text { lag1 constant } \\
-0.02940 .0701\end{array}$ & $\begin{array}{l}\text { Coefficients: } \\
\text { lag1 constant } \\
-0.0297 \quad 0.0715\end{array}$ \\
\hline $\begin{array}{l}\text { Degrees of freedom: } 133 \text { total; } 131 \\
\quad \text { residual } \\
\text { Residual standard error: } 0.1106\end{array}$ & $\begin{array}{l}\text { Degrees of freedom: } 133 \text { total; } 131 \\
\quad \text { residual } \\
\text { Residual standard error: } 0.1091\end{array}$ \\
\hline
\end{tabular}

Tabela 6.1 - Teste Dickey Fuller aumentado das séries de taxa de câmbio spot e futura

Para ambas as séries, pode-se observar que a hipótese nula não foi rejeitada ( $\mathrm{p}-$ valores 0,4921 e 0,4683), indicando a presença de uma raiz unitária nas séries. Resta saber se a relação entre elas resulta numa série estacionária para determinar se existe ou não cointegração.

Realizando os testes de regressão simples e regressão dinâmica apresentados no Capítulo 4, obtém-se os seguintes p-valores 6,306668e-007 (Tabela 6.2) e 0,0007594897 (Tabela 6.3), ou seja, rejeita-se a hipótese nula de não cointegração, como observado nas tabelas abaixo. 
Tabela 6.2 - Resultados do teste de cointegração por regressão simples

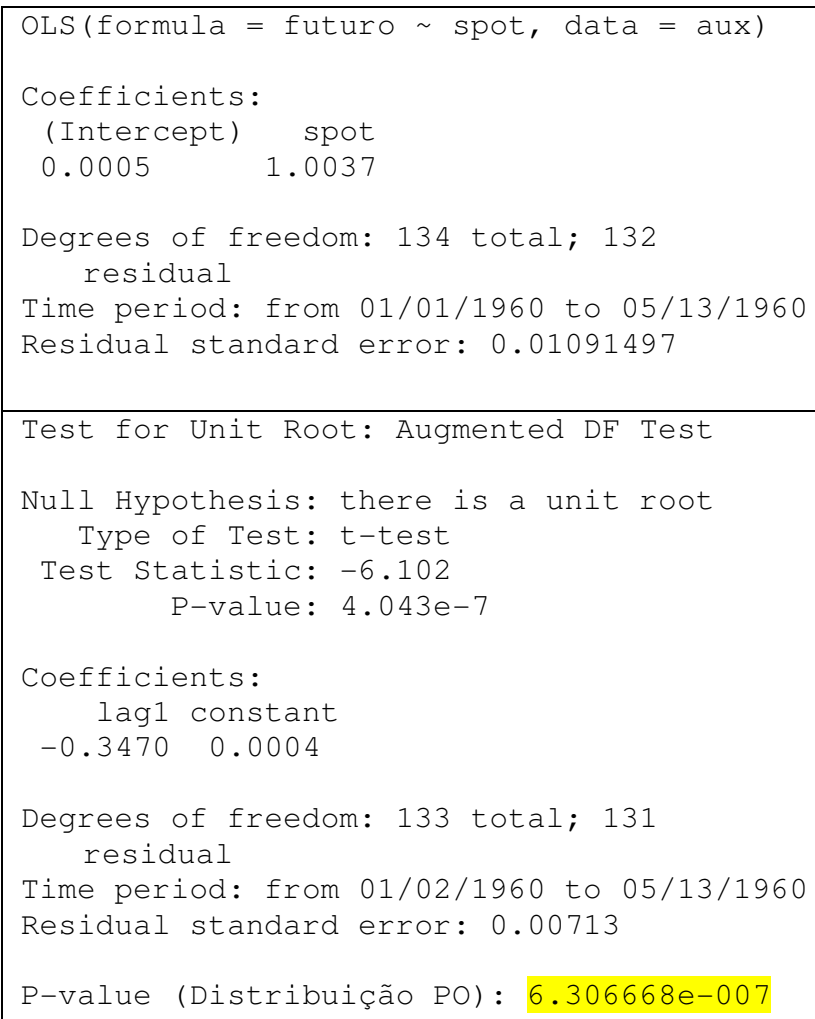


Tabela 6.3 - Resultados do teste de cointegração por regressão dinâmica

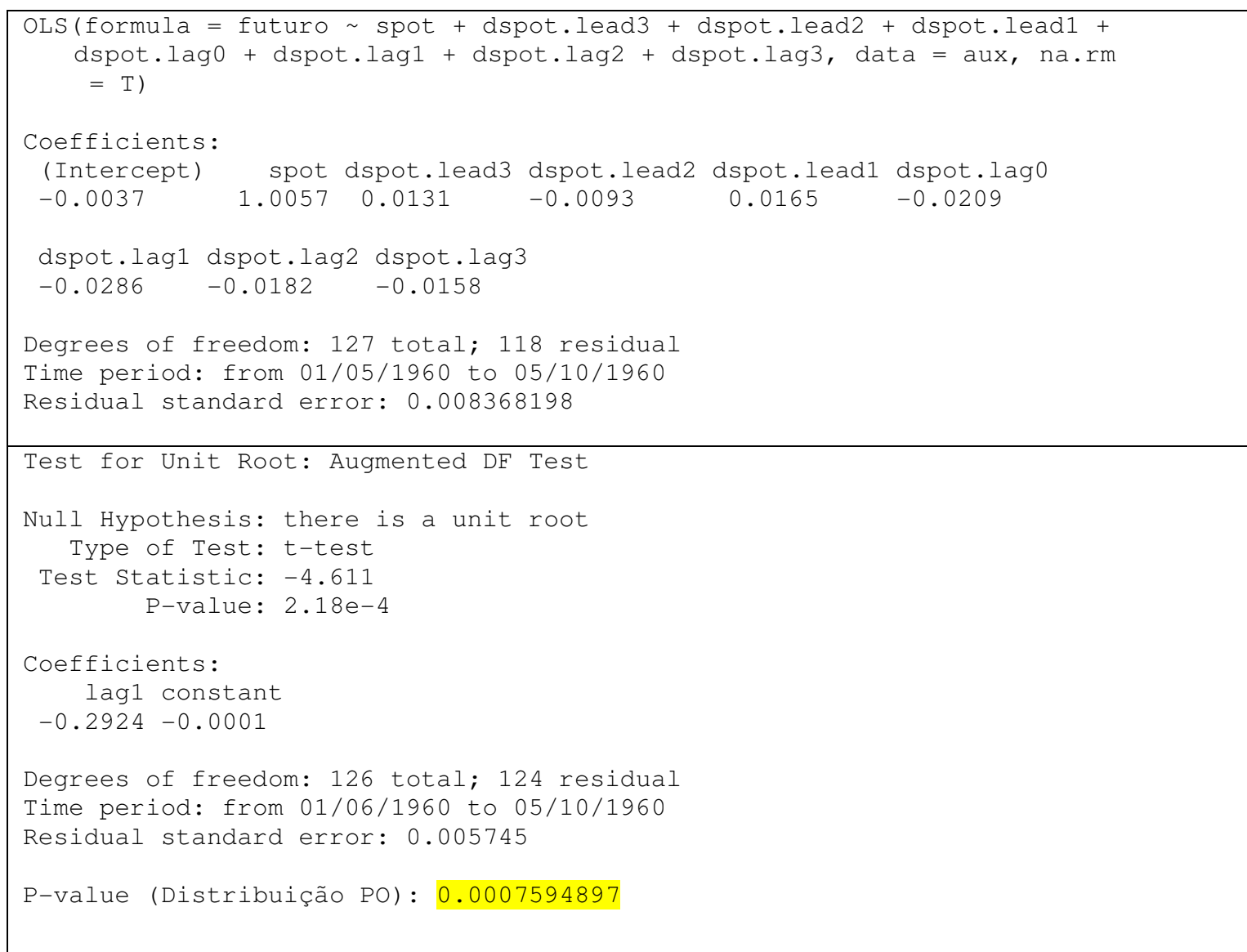

O resultado do teste de Johansen (Tabela 6.4) apresentou valores significantes a níveis de 5\% e 1\% para o fator de cointegração, apontando, também, para a existência de cointegração entre as séries.

Tabela 6.4 - Teste de Johansen sobre as séries de taxas de câmbio spot e futura

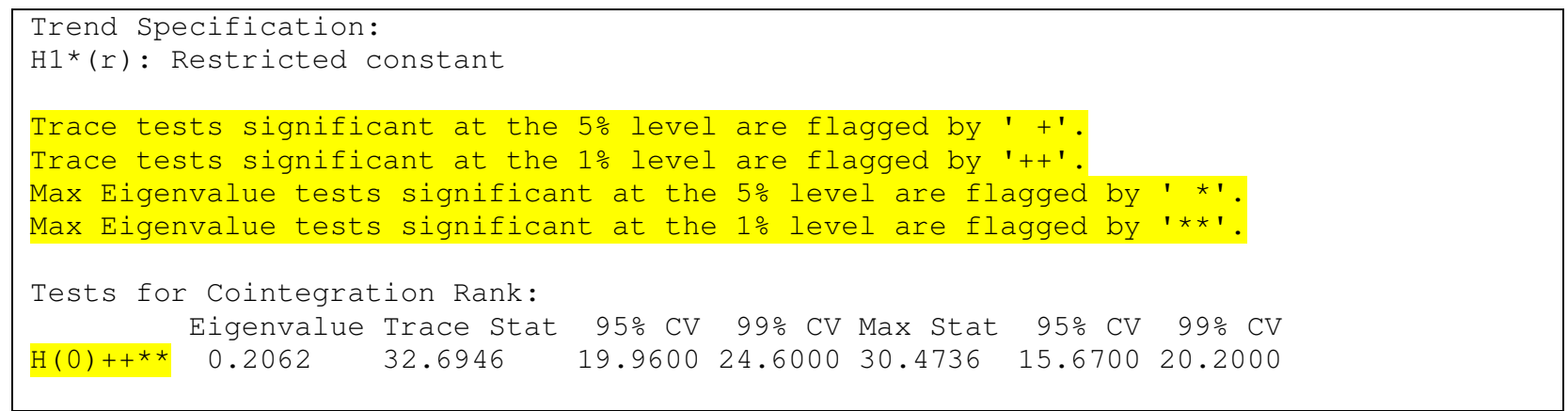

As estimativas para os vetores de cointegração podem ser observados na tabela abaixo, de onde percebe-se a consistência das estimativas. Como tanto o método DOLS quanto o de 
Johansen apresentaram ótimos resultados na maioria dos casos testados no capítulo anterior, ambos podem ser utilizados como estimativa.

Tabela 6.5 - vetores de cointegração das séries de taxas de câmbio spot e futura

\begin{tabular}{|l|c|}
\hline Método & $\widehat{\boldsymbol{\beta}}$ \\
\hline OLS & 1.0037 \\
\hline DOLS & 1.0057 \\
\hline Johansen & 1.0048 \\
\hline
\end{tabular}

Torna-se possível a obtenção, por regressão linear, do seguinte modelo de correção de erros entre as séries:

Tabela 6.6 - Estimação do modelo de correção de erros

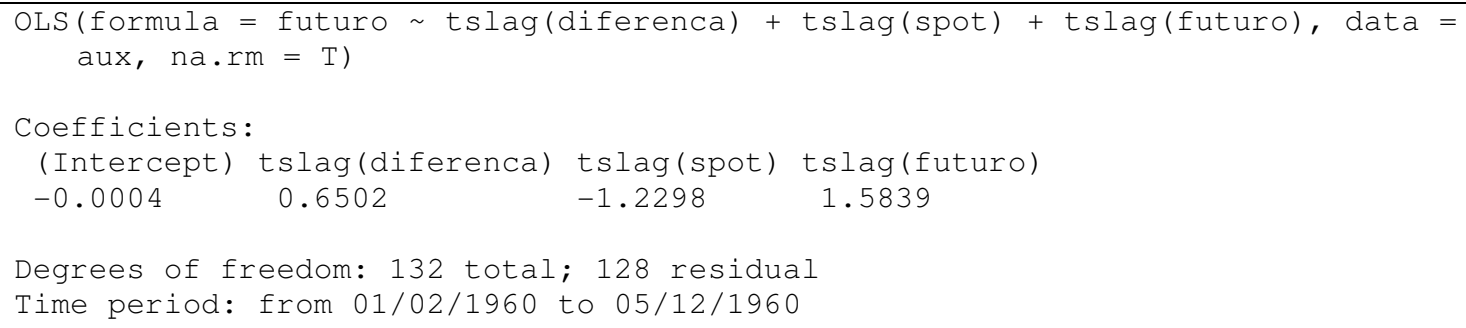

$$
\begin{aligned}
\Delta \text { futuro }_{t}=- & 0,0004+0,6502(\text { futuro }-1,0048 \text { spot })-1,2298 \Delta \text { spot }_{t-1} \\
& +1,5839 \Delta \text { futuro }_{t-1}+\varepsilon_{\mathrm{t}} .
\end{aligned}
$$

\subsection{Estimação do Modelo de Cointegração Fracionária}

Nesta seção, será efetuado o teste de existência de cointegração fracionária proposto por Davidson (2002) além da comparação dos resíduos deste modelo com os resíduos do modelo de cointegração fracionária para se determinar o que melhor se ajustou às séries observadas.

O primeiro passo para se determinar a existência de cointegração fracionária é o teste para presença de memória longa nas séries. Como observado no item anterior, as séries de preços de taxas de câmbio spot e futura apresentam FAC com lento decaimento, sugerindo a realização dos testes de memória longa.

O teste da estatística R/S abaixo rejeita a hipótese nula de não existência de dependência de longo prazo para ambas as séries, assim como o teste GPH que também rejeitou a hipótese nula de parâmetro $d=0$. 
Tabela 6.7 - Testes R/S e GPH para as séries de taxas de câmbio spot e futura

\begin{tabular}{|c|c|}
\hline Spot & Futuro \\
\hline $\begin{array}{l}\text { Test for Long Memory: Modified R/S Test } \\
\text { Null Hypothesis: no long-term dependence } \\
\text { Test Statistics: }\end{array}$ & $\begin{array}{l}\text { Test for Long Memory: Modified R/S Test } \\
\text { Null Hypothesis: no long-term dependence } \\
\text { Test Statistics: }\end{array}$ \\
\hline $2.1701 * \star$ & $2.1812 \star \star$ \\
\hline $\begin{array}{r}\star \text { : significant at } 5 \% \text { level } \\
\star * \text { : significant at } 1 \% \text { level }\end{array}$ & $\begin{array}{r}\text { * : significant at } 5 \% \text { level } \\
\star *: \text { significant at } 1 \% \text { level }\end{array}$ \\
\hline $\begin{aligned} \text { Total Observ }: & 134 \\
\text { Bandwidth }: & 4\end{aligned}$ & $\begin{aligned} \text { Total Observ. } & 134 \\
\text { Bandwidth }: & 4\end{aligned}$ \\
\hline $\begin{array}{l}\text { Test for Long Memory: GPH Test } \\
\text { Null Hypothesis: } d=0 \\
\text { Test Statistics: }\end{array}$ & $\begin{array}{l}\text { Test for Long Memory: GPH Test } \\
\text { Null Hypothesis: } d=0 \\
\text { Test Statistics: }\end{array}$ \\
\hline $\begin{aligned} \text { d } & 0.9219 \\
\text { stat } & 3.3729 * \star\end{aligned}$ & $\begin{aligned} \text { d } & 0.9499 \\
\text { stat } & 3.475^{* \star}\end{aligned}$ \\
\hline $\begin{array}{r}* \text { : significant at } 5 \% \text { level } \\
\star * \text { : significant at } 1 \% \text { level }\end{array}$ & $\begin{array}{r}\star \text { : significant at } 5 \% \text { level } \\
\star \star: \text { significant at } 1 \% \text { level }\end{array}$ \\
\hline
\end{tabular}

Comprovada a existência de dependência de longo prazo, o próximo passo é a estimação do parâmetro de memória longa $d$. Como foi constatado no item anterior a presença de uma raiz unitária, a estimação do parâmetro $d$ será efetuada sobre a série em primeiras diferenças.

As estimativas obtidas pelos diferentes métodos para as séries em primeiras diferenças podem ser observadas na tabela abaixo.

Tabela 6.8 - Estimativas do parâmetro de memória longa

\begin{tabular}{|l|c|c|}
\hline Método & d.spot & d.futuro \\
\hline Whittle & 0.27509 & 0.28858 \\
\hline Periodograma & 0.067780 & 0.073097 \\
\hline R/S & 0.22069 & 0.22525 \\
\hline EMV & 0.2649 & 0.2722 \\
\hline GPH & -0.1246 & -0.3657 \\
\hline
\end{tabular}

Como visto no Capítulo 3, o estimador de máxima verossimilhança foi o que mais se aproximou dos valores reais e, por este motivo, foi escolhido como estimativa. Tomada a diferença fracionária dos dados, observa-se os seguintes resultados de FAC e PACF. 
Series : spot.farima\$residuals

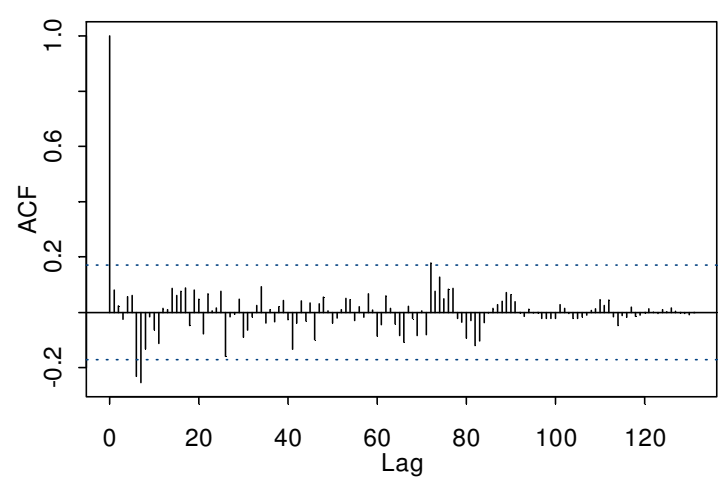

Series : futuro.farima $\$$ residuals

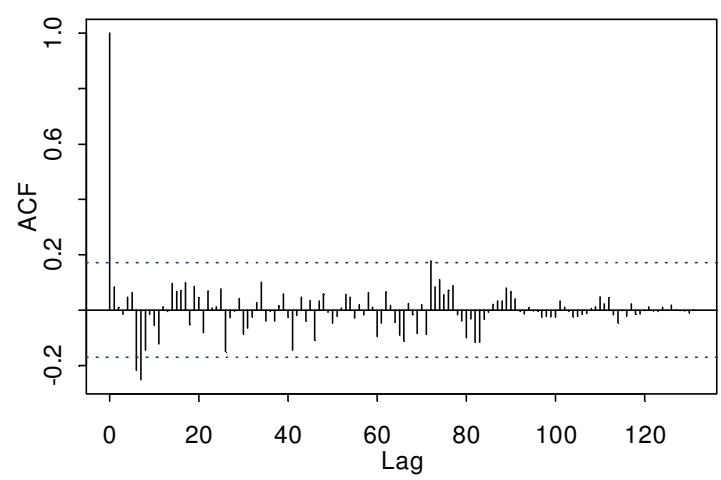

Series : spot.farima\$residuals

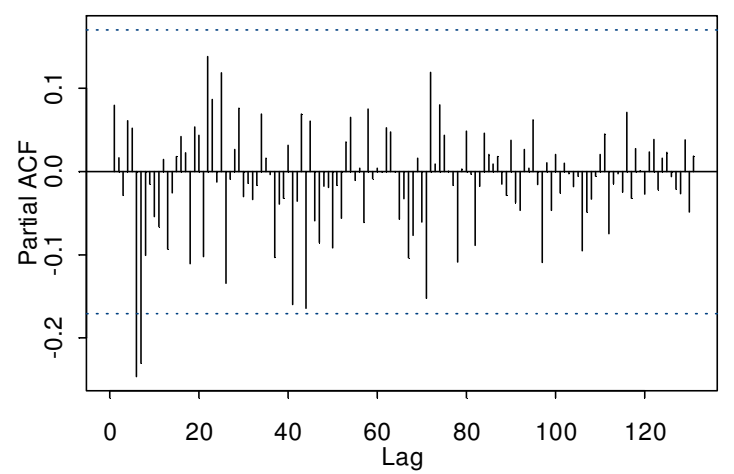

Series : futuro.farima $\$$ residuals

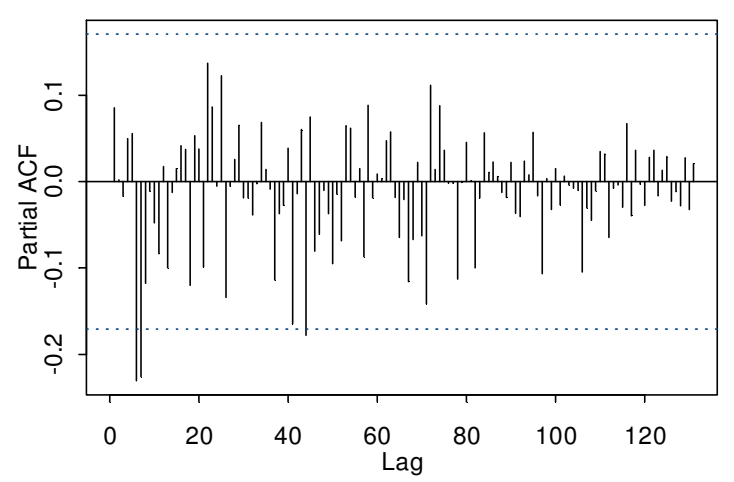

Figura 6.3 - FAC e FACP dos resíduos das séries em diferenças fracionárias pelo parâmetro de memória longa

Pelos gráficos da Figura 6.3, observa-se a presença de correlações significativas entre defasagens de ordens 6 e 7 que, quando eliminadas, geram o seguinte modelo:

$$
\begin{gathered}
\left(1+0,2192 \mathrm{~B}^{6}+0,2479 \mathrm{~B}^{7}\right)(1-B)^{1,2649} \text { spot }_{t}=a_{t} \\
\left(1+0,2020 \mathrm{~B}^{6}+0,2453 \mathrm{~B}^{7}\right)(1-B)^{1,2722} \text { futuro }_{t}=a_{t}
\end{gathered}
$$

cujos resíduos apresentam os seguintes gráficos de FAC e FACP abaixo, onde pode-se observar a eliminação das auto correlações significativas. 
Series : spot\$residuals

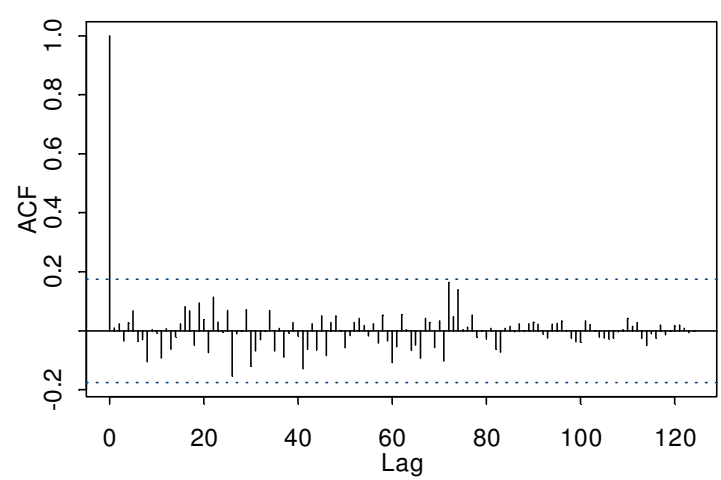

Series : futuro\$residuals

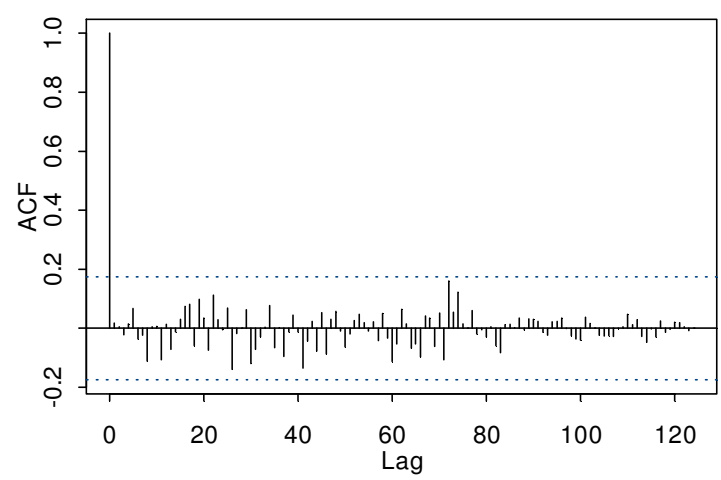

Series : spot\$residuals

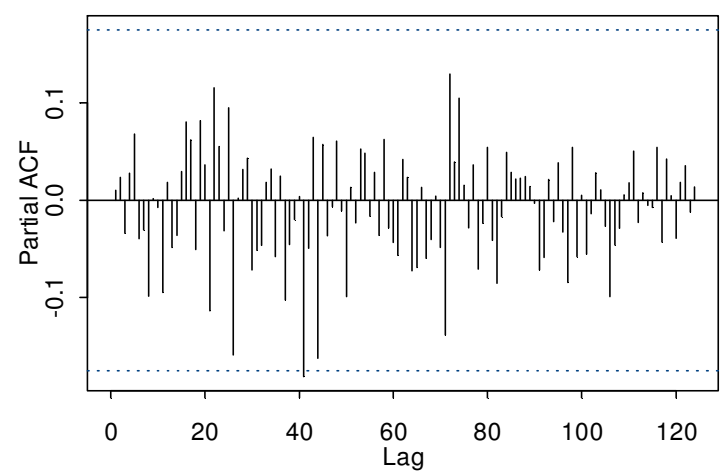

Series : futuro\$residuals

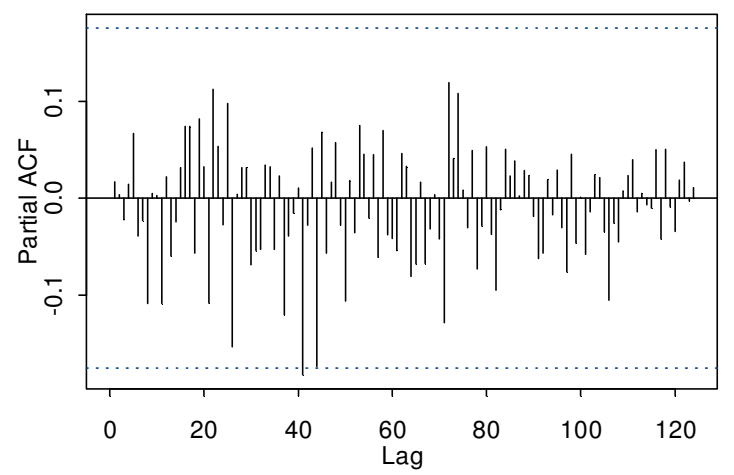

Figura 6.4 - FAC e FACP dos resíduos dos modelos ajustados

Efetuando-se os testes de cointegração fracionária com 500 reamostragens de bootstrap os seguintes resultados podem ser observados.

Tabela 6.9 - p-valores dos testes de cointegração fracionária sobre os dados reais

\begin{tabular}{|c|c|c|c|}
\hline Teste & Hip.Forte & Hip.SemiFraca & Hip.Fraca \\
\hline FEG & $0.00 \%$ & $0.00 \%$ & $0.00 \%$ \\
\hline DW & $0.00 \%$ & $0.00 \%$ & $0.00 \%$ \\
\hline
\end{tabular}

Todos os testes rejeitam a hipótese nula de independência estatística (hipótese forte) indicando a dependência entre as séries. As hipóteses nulas de não cointegração e inexistência de causalidade de Granger (hipótese semifraca) e de simples não cointegração (hipótese fraca) também foram rejeitadas pelos testes indicando a existência de cointegração fracionária. Os cálculos dos testes FEG e DW utilizando os resíduos da regressão entre as séries originais, taxa 
de câmbio spot e futura, resultaram em 0.4827 e 0.6933 , respectivamente e os gráficos abaixo apresentam as distribuições das estatísticas obtidas com os resíduos das regressões entre as séries reamostradas.

Figura 6.5 - Distribuições das diferentes hipóteses das estatísticas simuladas

\begin{tabular}{|c|c|c|c|c|c|c|}
\hline & \multicolumn{2}{|r|}{ Hip.Forte } & \multicolumn{2}{|r|}{ Hip.SemiFraca } & \multicolumn{2}{|r|}{ Hip.Fraca } \\
\hline FEG & \begin{tabular}{|l|}
$70 \%$ \\
$60 \%$ \\
$50 \%$ \\
$40 \%$ \\
$30 \%$ \\
$20 \%$ \\
$10 \%$ \\
$0 \%$ \\
\end{tabular} & $\mid$\begin{tabular}{|l||}
$\mid$ \\
\end{tabular} & $\begin{array}{c}70 \% \\
60 \% \\
50 \% \\
40 \% \\
30 \% \\
20 \% \\
10 \% \\
0 \% \\
\end{array}$ & \begin{tabular}{|c|c|} 
& \\
& \\
& \\
& \\
\end{tabular} & \begin{tabular}{l|l|}
$70 \%$ \\
$60 \%$ \\
$50 \%$ \\
$40 \%$ \\
$30 \%$ \\
$20 \%$ \\
$10 \%$ \\
$0 \%$ \\
\end{tabular} & $\mid \frac{||}{||}$ \\
\hline DW & $\begin{array}{c}50 \% \\
45 \% \\
40 \% \\
35 \% \\
30 \% \\
25 \% \\
20 \% \\
15 \% \\
10 \% \\
5 \% \\
0 \% \\
\end{array}$ & || $\mid$ & $\begin{array}{c}50 \% \\
45 \% \\
40 \% \\
35 \% \\
30 \% \\
25 \% \\
20 \% \\
15 \% \\
10 \% \\
5 \% \\
0 \% \\
\\
\end{array}$ & ||$_{\mid}^{\mid}$ & $\begin{array}{l}50 \% \\
45 \% \\
40 \% \\
35 \% \\
30 \% \\
25 \% \\
20 \% \\
15 \% \\
10 \% \\
5 \% \\
0 \% \\
\end{array}$ & |||| \\
\hline
\end{tabular}

Por regressão linear, o seguinte modelo de correção de erros pode ser obtido:

Tabela 6.10 - Estimação do modelo de Correção de Erros Fracionário

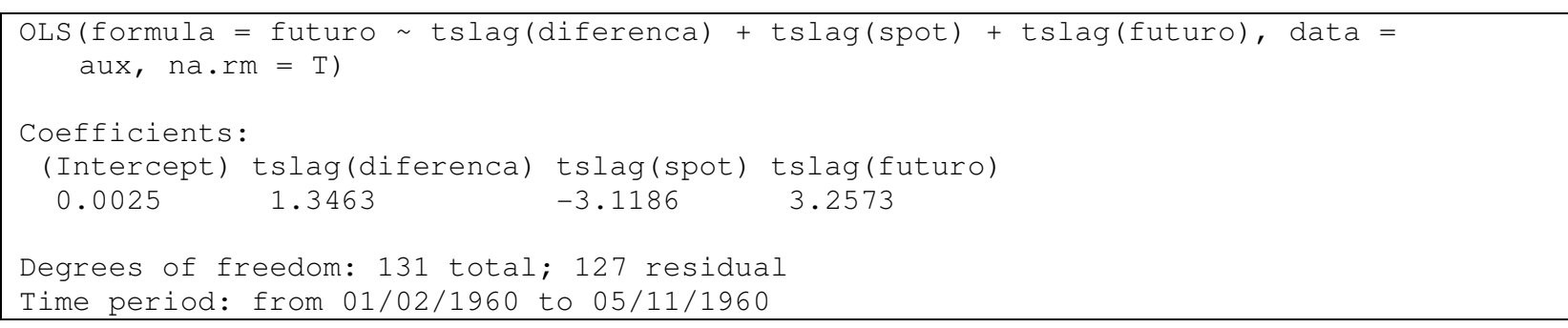

$$
\Delta^{1.2722} \text { futuro }_{t}=-0,0025+1,3463(\text { futuro }-1,0048 s p o t)-3,1186 \Delta^{1,2649} \text { spot }_{t-1}+
$$
$3,2573 \Delta^{1,2722}$ futuro $_{t-1}+\varepsilon_{\mathrm{t}}$. 
Pelo modelo observamos que as séries buscam uma relação de equilíbrio próxima de um, ou seja, equivalência entre as duas séries. Além disso, a tendência para a relação de equilíbrio é corrigida com fator de velocidade elevado.

O erro quadrático médio do modelo de cointegração encontrado foi de 0,009267 enquanto para o modelo de correção de erros fracionário o valor encontrado foi de 0,009001 indicando que o modelo fracionário ajustou-se melhor às séries observadas.

Embora o modelo de cointegração fracionária tenha se ajustado melhor às séries, observa-se que o custo computacional foi muito elevado e a diferença no valor do erro quadrático médio foi pequena. Em situações como esta, um procedimento ótimo para encontrar a relação de longo prazo entre as séries seria: estimar o modelo de cointegração convencional, sendo encontrada a presença de cointegração e estimada a relação de longo prazo terminar o procedimento; caso não serem encontrados indícios de cointegração e acreditar-se na existência de relações entre as séries, realizar os testes de cointegração fracionária. 


\section{Capítulo 7 Conclusão}

Ambos os testes de cointegração $I(1)$ e cointegração fracionária apresentaram poderes de teste bastante elevados para altas ordens de integração. Os procedimentos de estimação dos valores do vetor de cointegração por mínimos quadrados e por máxima verossimilhança convergiram na maioria dos casos salvo aqueles em que o parâmetro de velocidade era baixo.

O procedimento de teste de cointegração fracionária mostrou-se lento para 500 reamostragens de bootstrap de séries de 500 observações. Como Davidson (2002) recomenda no mínimo a utilização de 500 reamostragens de bootstrap a única variável que pode ser alterada é o número de valores observados. Mesmo com apenas 50 simulações de cada modelo, é possível observar a consistência do método.

Os testes utilizando dados reais mostrou que tanto o modelo de cointegração convencional quanto o de cointegração fracionária são aplicáveis em algumas séries brasileiras. Davidson (2002) observou em seu trabalho que os testes de cointegração fracionária comportam até mesmo o caso de séries cujo parâmetro de integração é unitário, comprovado através das simulações dos modelos ARIMA, cointegrados e não cointegrados no Capítulo 5.

Trabalhos futuros sobre o tema envolvem a diminuição do tempo de execução do teste de cointegração fracionária através de algoritmos alternativos, possibilitando a simulação de um número maior de amostras para avaliação dos poderes dos testes, em que poderia ser observada a convergência para o valor de nível do teste, quando simuladas séries não cointegradas. Outro ponto adicional seria a inclusão da estatística de ajuste dos modelos (Goodness of Fit) entre os testes de cointegração fracionária que durante as simulações realizadas neste trabalho não convergiu para resultados esperados. 


\section{Apêndice A Referências Bibliográficas}

[1] Alexander, C. (2001). Market Models. Wiley.

[2] Baillie, R. T., Bollerslev, T. (1994). Cointegration, Fractional Integration and exchange rate Dynamics. Journal of Finance XLIX (2), 737-745.

[3] Box, G. E. P., Jenkins, G. M. and Reinsel, G. (1994). Time Series Analysis: Forecasting and Control. Third Edition. Prentice Hall, Englewood Cliffs.

[4] Caporale, G. M., Gil-Alana, L. A. (2004). Fractional Cointegration and Test of Present Value Models. Review of Financial Economics, 13, 245-258.

[5] Cheung, Y. M., Lai, K. S. (1993). A Fractional Cointegration Analysis of Purchasing Power Parity. Journal of Business and Economic Statistics 11(1), 103-112.

[6] Davidson, J. (2002). A Model of Fractional Cointegration and Tests for Cointegration Using the Bootstrap. Journal of Econometrics, 110, 2, 187-212.

[7] Dickey, D., W. Fuller (1979). Distribution if the Estimators for Autoregressive Time Series with a Unit Root. Journal of the American Statistical Association, 74, 427-431.

[8] Engle, R. F., Granger, C. W. J. (1987). Cointegration and Error Correction: Representation, Estimation and Testing. Econometrica, 55, 251-276.

[9] Fox, A. J., Taqqu, M. S. (1986). Large Sample Properties of Parameter Estimates for Strongly Dependent Stationary Gaussian Time Series. The Annals of Statistics, 14, 517-532.

[10] Geweke, J., Porter-Hudak, S. (1983). The Estimation and Application of Long Memory Time Series Models. Journal of Time Series Analysis, 4, 221-237.

[11] Granger, C. W. J. (1969). Investigating causal relationships by econometric models and cross-spectral methods. Econometrica, 37, 424-438.

[12] Granger, C. W. J. and Newbold, P. E. (1974). Spurious regression in econometrics. Journal of Econometrics, 2, 111-120. 
[13] Hannan, E. J. (1973). The Asymptotic Theory of Linear Time Series Models. Journal of Applied Probability, 10, 130-145.

[14] Hurst, H. E. (1951). Long Term Storage Capacity of Reservoirs. Transactions of the American Society of Civil Engineers, 116, 770-799.

[15] Johansen, S. (1988). Statistical Analysis of Cointegration Vectors. Journal of Economic Dynamics and Control, 12, 231-254.

[16] Johansen, S. (1995). Likelihood Based Inference in Cointegrated Vector Error Correction Models. Oxford University Press, Oxford.

[17] Lo, A. W. (1991). Long Term Memory in Stock Market Prices. Econometrica, 59, 12791313.

[18] Madelbrot, B. B. (1975). Limit Theorems on the Self-Normalized Range for Weakly and Strongly Dependent Processes. Zeitschrift für Wahrscheinlichkeitstheorie und Verwandte Gebiete, 31, 271-285.

[19] Marmol, F. (1998). Spurious Regression Theory with Nonstationary Fractionally Integrated Processes of Different Orders. Oxford Bulletin of Economics and Statistics, 84, 233-250.

[20] Marques, G. O. (2007). Estruturas de memória longa em variáveis econômicas: da análise de integração e co-integração fracionária à análise de ondaletas. Tese de Mestrado. FEA - USP, São Paulo.

[21] Morettin, P. A. (2008). Econometria Financeira. Edgar Blücher, São Paulo.

[22] Morettin, P. A., Toloi, C. M. C. (2004). Análise de Séries Temporais. Edgar Blücher, São Paulo.

[23] Newey, W. K., West, K. D. (1987). A Simple Positive Semidefinite Heteroskedasticity and Autocorrelation Consistent Covariance Matrix. Econometrica, 55, 703-708. 
[24] Osterwald-Lenum, M. (1992). A note with Quantiles of the Asymptotic Distribution of the Maximum Likelihood Cointegration Rank Statistics. Oxford Bulletin of Economics and Statistics, 54, 311-340.

[25] Phillips, P.C.B. (1991). Optimal Inference in Cointegrated Systems. Econometrica, 59, 283-306.

[26] Phillips, P. C. B., S. Ouliaris (1990). Asymptotic Properties of Residual Based Tests for Cointegration. Econometrica, 58, 73-93.

[27] Robinson P.M., Marinucci D. (1998). Semiparametric Frequency Domain Analysis of Fractional Cointegration. STICERD Working Paper EM/98/348.

[28] Stock, J. H. (1987). Asymptotic Properties of Least Squares Estimation of Cointegrating Vectors. Econometrica, 55, 1035-1056.

[29] Stock, J. H., M. W. Watson (1993). A Simple Estimator of Cointegrating Vectors in Higher Order Integrated Systems. Econometrica,61, 783-820.

[30] Tsay, R. S. (2005). Analysis of Financial Time Series. Second Edition. Second Edition. Wiley, New York.

[31] Zivot, E., Wang, J. (2005). Modeling Financial Time Series with S-Plus. Second Edition. Springer, New York. 


\section{Apêndice B Comandos Splus}

A lista abaixo apresenta os comandos utilizados e uma breve apresentação.

simulate.FARIMA Simula séries temporais que seguem modelos ARFIMA.

FARIMA Ajusta modelos ARFIMA a uma série temporal.

d.pgram Estima parâmetro de integração $d$ de memória longa pelo método do periodograma.

d.whittle Estima parâmetro de integração $d$ de memória longa pelo método do Whittle.

d.ros Estima parâmetro de integração $d$ de memória longa pelo método R/S.

gphTest Teste e estimador do parâmetro de memória longa pelo método GPH.

rmvnorm Simula séries temporais seguindo distribuições normais.

ols.fit função de regressão linear.

unitroot teste de raiz unitária.

pcoint Estima p-valor do teste de raiz unitária pela distribuição de PO.

arima.sim Simula séries temporais que seguem o modelo ARIMA.

acf funções de auto correlação e auto correlação parcial

coint teste de cointegração de Johansen. 